Florida International University FIU Digital Commons

$11-4-2016$

\title{
A Framework for Recommending Signal Timing Improvements Based on Automatic Vehicle Matching Technologies
}

Xuanwu Chen

Florida International University, xchen015@fiu.edu

DOI: 10.25148 /etd.FIDC001219

Follow this and additional works at: https://digitalcommons.fiu.edu/etd

Part of the Civil Engineering Commons, and the Transportation Engineering Commons

\section{Recommended Citation}

Chen, Xuanwu, "A Framework for Recommending Signal Timing Improvements Based on Automatic Vehicle Matching Technologies" (2016). FIU Electronic Theses and Dissertations. 3003.

https://digitalcommons.fiu.edu/etd/3003 


\title{
FLORIDA INTERNATIONAL UNIVERSITY
}

Miami, Florida

\section{A FRAMEWORK FOR RECOMMENDING SIGNAL TIMING IMPROVEMENTS BASED ON AUTOMATIC VEHICLE MATCHING TECHNOLOGIES}

\author{
A dissertation submitted in partial fulfillment of \\ the requirements for the degree of \\ DOCTOR OF PHILOSOPHY \\ In \\ CIVIL ENGINEERING
}

by

Xuanwu Chen

2016 
To: Interim Dean Ranu Jung

College of Engineering and Computing

This dissertation, written by Xuanwu Chen, and entitled A Framework for Recommending Signal Timing Improvements Based on Automatic Vehicle Matching Technologies, having been approved in respect to style and intellectual content, is referred to you for judgement.

We have read this dissertation and recommend that it be approved.

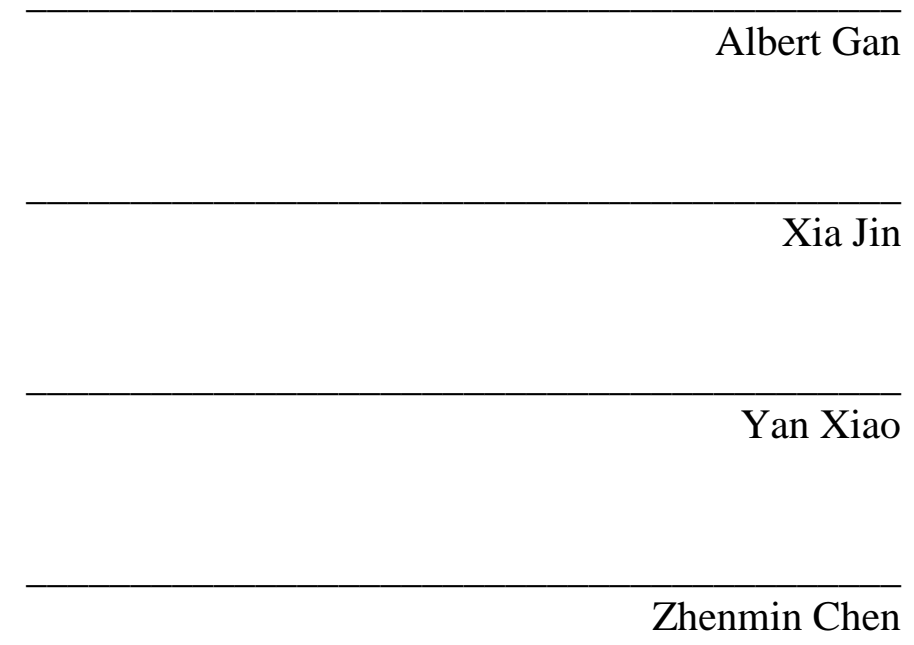

Zhenmin Chen

Mohammed Hadi, Major Professor

Date of Defense: November 4, 2016

The dissertation of Xuanwu Chen is approved.

Interim Dean Ranu Jung College of Engineering and Computing

Andrés G. Gil

Vice President for Research and Economic Development

And Dean of the University Graduate School

Florida International University, 2016 
(C) Copyright 2016 by Xuanwu Chen

All rights reserved. 


\section{DEDICATION}

I dedicate this dissertation to my beloved wife, Fan, my parents, Yunhui and Ke, and my son, Ethan, for their forever love and endless support. 


\section{ACKNOWLEDGMENTS}

I am so fortunate to have an excellent advisor, Dr. Mohammed Hadi, to guide me throughout my adventure of academic enlightenment. This dissertation would not have come to completion without his motivation, guidance, and support. I express my profound gratitude for his inspirational mentorship and invaluable advice. He has always been like a lighthouse to me, guiding me in both my professional career and personal life.

I am also honored to have Dr. Albert Gan, Dr. Xia Jin, Dr. Yan Xiao, and Dr. Zhenmin Chen serving as my dissertation committee. I would like to express my deep appreciation to each of them for their insightful feedback, scholarly advice, and continuous support. Special thanks go to Dr. Yan Xiao, who provided me with abundant and precious advice. I would also like to thank Dr. L. David Shen for his encouragement and suggestions. I sincerely appreciate Dr. Tao Wang for his technical support on programming.

I am truly grateful to Dr. Ming S. Lee, Dr. Halit Ozen, Dr. Priyanka Alluri, Dr. Wanyang Wu, Dr. Jinyan Lu, Mr. Jianmin Jia, Mr. Hiram Hernandez, and Dr. Dibakar Saha, for their generous help during my doctoral studies. I would also like to thank Ms. Natalie Defraene for the editing of this dissertation. In addition, I would like to extend my gratitude to all other faculty and staff in the department for their continual motivation as I achieved my academic and professional goals.

Finally, I would like to thank my wife, Dr. Fan Liu for her love, patience, and sacrifices. She waited for my return to Orlando from Miami every week for the past five years. I also want to express my deep appreciation to my parents for their understanding and support for my journey overseas. I would like to extend my gratitude to my parentsin-law, who always care about my scholarly accomplishments. 


\title{
ABSTRACT OF THE DISSERTATION \\ A FRAMEWORK FOR RECOMMENDING SIGNAL TIMING IMPROVEMENTS BASED ON AUTOMATIC VEHICLE MATCHING TECHNOLOGIES
}

\author{
by \\ Xuanwu Chen \\ Florida International University, 2016 \\ Miami, Florida

\section{Professor Mohammed Hadi, Major Professor}

Continuously monitoring and automatically identifying existing problems in traffic signal operation is a challenging and time-consuming task. Although data are becoming available due to the adoption of emerging detection technologies, efforts on utilizing the data to diagnose signal control are limited. The current practices of retiming signals are still periodic and based on several days of aggregated turning movement counts. This dissertation developed a framework of automatic signal operation diagnosis with the aim to support decision-making processes by assessing the signal control and identifying the signal retiming needs. The developed framework used a combination of relatively low-cost data from Wi-Fi sensors and historical signal timing records from existing signal controllers.

The development involved applying multiple data matching and filtering algorithms to allow the estimation of travel times of vehicular traversals. The Travel Time Index (TTI) was then used as a measure to assess the traffic conditions of various movements. Historical signal timing records were also analyzed, and an additional signal- 
timing measure, referred to as the Max-out Ratio $(M R)$, was proposed to evaluate the frequency in which the green time demand of a phase exceeded its preset value.

Thresholds for the $T T I$ and $M R$ variables were used as a basis for the diagnosis. This diagnosis first identified the needs for assigning additional green times for individual signal phases. Further assessments were then made to determine whether or not the cycle length for the entire intersection or capacity was sufficient.

The developed framework was implemented in a real-world signalized intersection and proved to be capable of identifying retiming needs, as well as providing support for the retiming process. Compared to field observations, the diagnosis results were able to reflect the signal operations of most of the movements during various time periods. Moreover, the flexibility of the developed framework allows users to select different thresholds for various movements and times of day, and thus customize the analysis to agency needs. 


\section{TABLES OF CONTENTS}

CHAPTER

PAGE

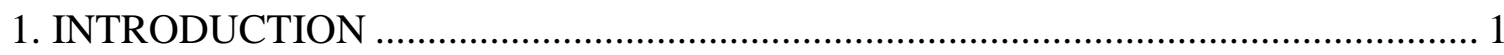

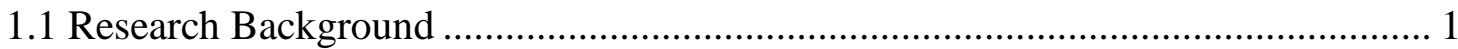

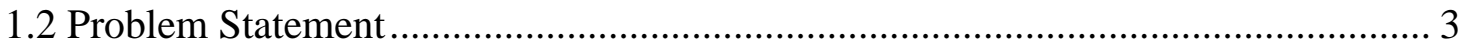

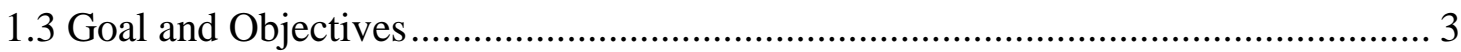

1.4 Dissertation Organization ............................................................................... 4

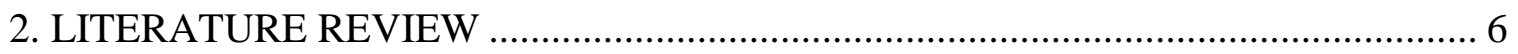

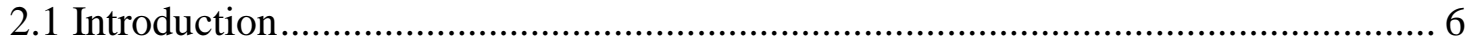

2.2 Automated Data Collection and Utilization for Traffic Signal Operation Diagnosis6

2.2.1 Automated Data Collection and Performance Measure Generation................... 7

2.2.2 Automated Signal Diagnosis Attempts ........................................................... 11

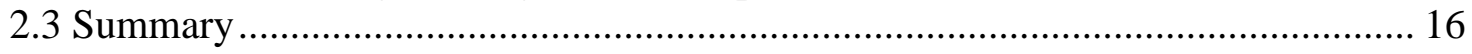

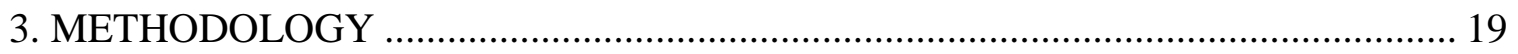

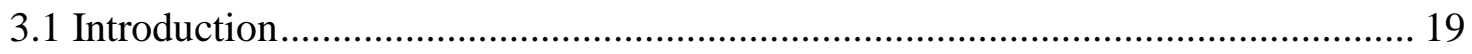

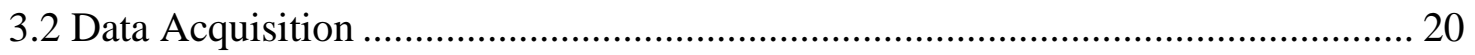

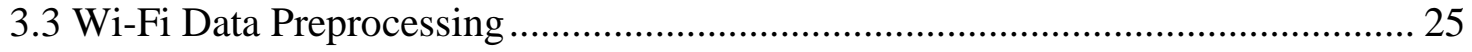

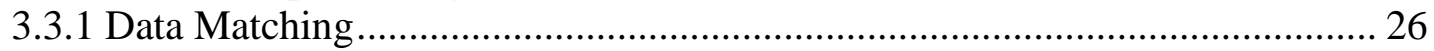

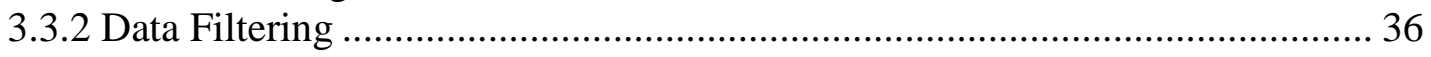

3.3.3 Data Aggregation (Travel Time Representation)............................................. 47

3.4 Signal Data Preprocessing ......................................................................... 55

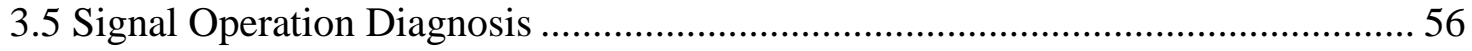

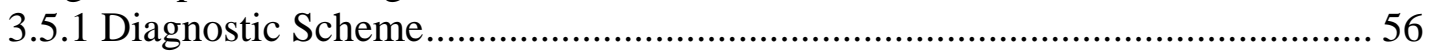

3.5.2 Individual Phase Diagnosis ......................................................................... 59

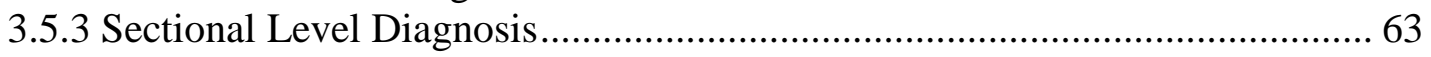

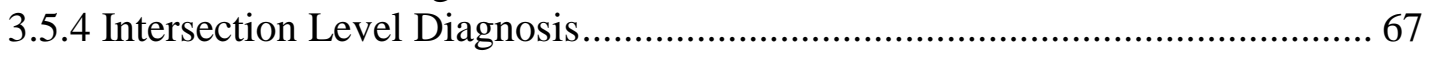

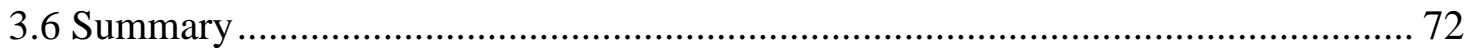

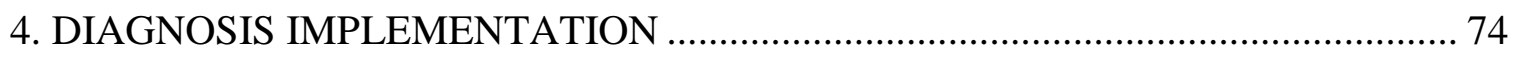

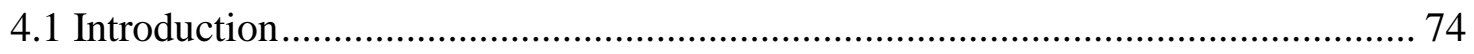

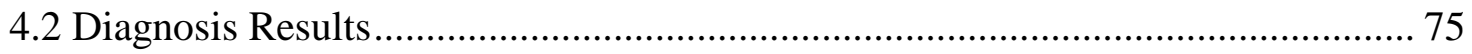

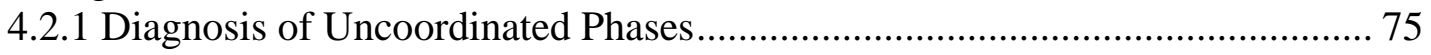

4.2.2 Diagnosis of Coordinated Phases................................................................. 80

4.2.3 Diagnosis of Intersection Cycle Length............................................................ 81

4.3 Comparison of Diagnoses and Field Observations .............................................. 85

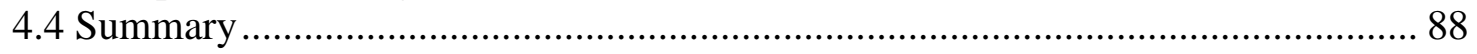

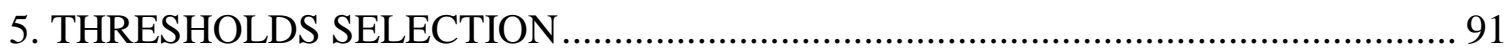

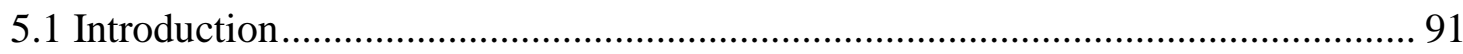

5.2 Comparison of Thresholds ................................................................................ 91

5.3 Comparison of the Diagnosis Results ............................................................... 92 
5.4 Summary

6. SUMMARY, CONCLUSIONS, AND RECOMMENDATIONS ............................. 99

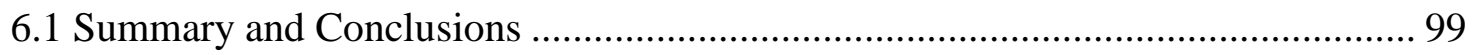

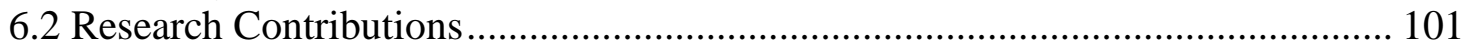

6.3 Recommendations for Future Research ........................................................... 101

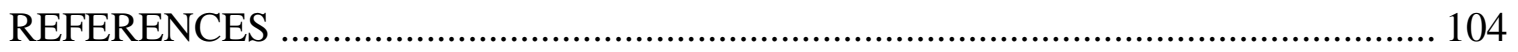

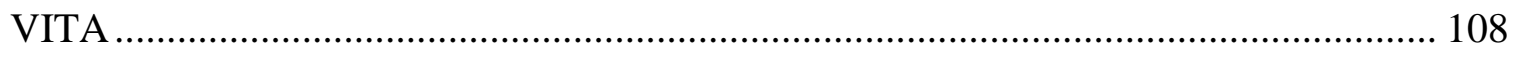




\section{LIST OF TABLES}

TABLE

PAGE

Table 3-1 Wi-Fi Detection Raw Data Example ...................................................... 23

Table 3-2 Example of Multiple Trips Made by One Device ......................................... 27

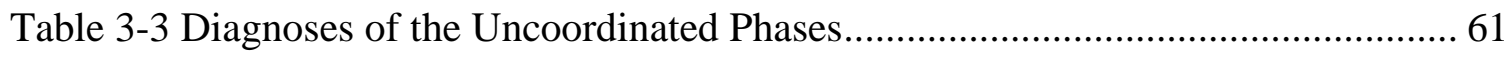

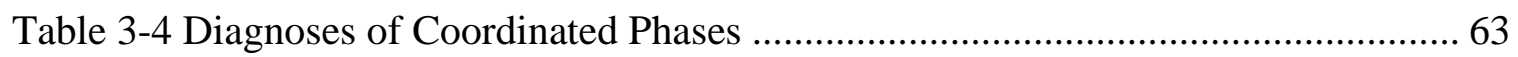

Table 4-1 Summary of Queue Levels from Field Observation.................................... 86

Table 4-2 Summary of Movement Blockages from Field Observation.......................... 87

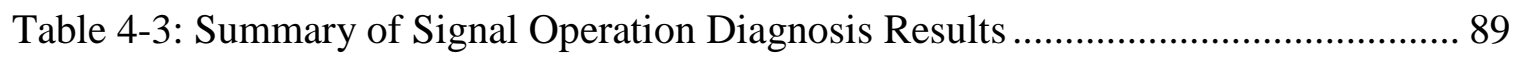

Table 5-1 Thresholds Utilized in Different Implementations ...................................... 92 


\section{LIST OF FIGURES}

FIGURE

PAGE

Figure 2-1: Typical Dual-Ring Eight-Phase Diagram ........................................... 13

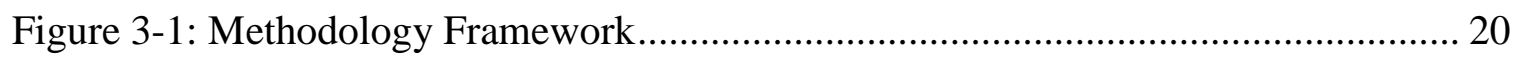

Figure 3-2 Wi-Fi Sensors Installed inside the Traffic Signal Controller Cabinet ........... 22

Figure 3-3 Wi-Fi Detector Locations (Background image source: Map data (C) 2016

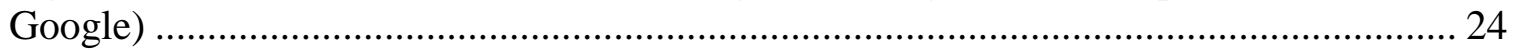

Figure 3-4 Example of KITS $®$ Historical Intersection Timing Report ......................... 25

Figure 3-5 Data Matching between Studied Intersection and Its Surrounding

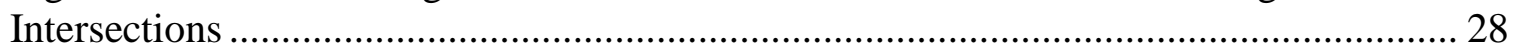

Figure 3-6 Example of Correct Matches and Mismatches for a MAC Address from

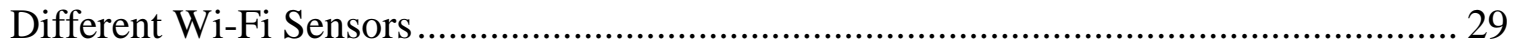

Figure 3-7 Example of Correct Matches and Mismatches with Actual Data .................. 30

Figure 3-8 Illustration of Different Types of Matched Travel Times............................ 35

Figure 3-9 Example Travel Times with Application of Rule 1 to Rule 5....................... 38

Figure 3-10 Example Travel Times with Artificial Dividing Line .............................. 39

Figure 3-11 Clusters Identified by DBSCAN Algorithm ....................................... 41

Figure 3-12 Identified Traversals and Other trips on the NBT Movement .................... 43

Figure 3-13 Identified Traversals and Other trips on the EBL Movement...................... 43

Figure 3-14 Identified Traversals and Other trips on the EBT Movement ..................... 44

Figure 3-15 Identified Traversals and Other trips on the NBL Movement .................... 44

Figure 3-16 Identified Traversals and Other trips on the WBL Movement ................... 45

Figure 3-17 Identified Traversals and Other trips on the WBT Movement .................... 45

Figure 3-18 Identified Traversals and Other trips on the SBL Movement ..................... 46

Figure 3-19 Identified Traversals and Other trips on the SBT Movement ..................... 46 
Figure 3-20 Cumulative Density Functions by Hours for Eastbound Left-turn Movement 51

Figure 3-21 Cumulative Density Functions by Hours for Eastbound Movements........... 52

Figure 3-22 Cumulative Density Functions by Hours for Westbound Movements. ........ 52

Figure 3-23 Cumulative Density Functions by Hours for Northbound Movements. ....... 53

Figure 3-24 Cumulative Density Functions by Hours for Southbound Movements. ....... 54

Figure 3-25 Time-of-Day Signal Timing Plan Schedule Report for the Intersection of Southwest 8th Street at Southwest 107th Avenue ........................................................ 55

Figure 3-26 Proposed Decision-Support Signal Operation Diagnostic Scheme ……....... 58

Figure 3-27 Typical Dual-Ring-Eight-Phase Signal Diagram for the Signal Control at

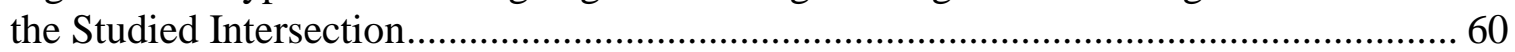

Figure 3-28 Illustration of Signal Phase Sections in the Phase Diagram .......................... 65

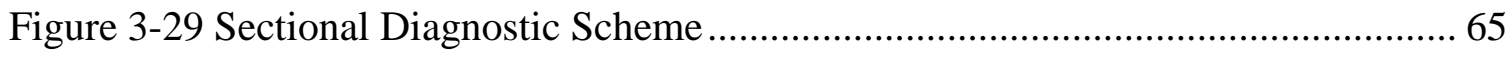

Figure 3-30 Combinations While Intersection Level Diagnosis Results are "1" ............. 70

Figure 3-31 Combinations While Intersection Level Diagnosis Results are “-1" ............ 71

Figure 4-1 Uncoordinated Signal Phase Diagnosis for EBL Movement........................... 77

Figure 4-2 Uncoordinated Signal Phase Diagnosis for NBL Movement ………............. 78

Figure 4-3 Uncoordinated Signal Phase Diagnosis for NBT Movement ......................... 78

Figure 4-4 Uncoordinated Signal Phase Diagnosis for SBL Movement ........................... 79

Figure 4-5 Uncoordinated Signal Phase Diagnosis for SBT Movement .......................... 79

Figure 4-6 Uncoordinated Signal Phase Diagnosis for WBL Movement.......................... 80

Figure 4-7 Coordinated Signal Phase Diagnosis for EBT Movement ................................ 81

Figure 4-8 Coordinated Signal Phase Diagnosis for WBT Movement.............................. 81

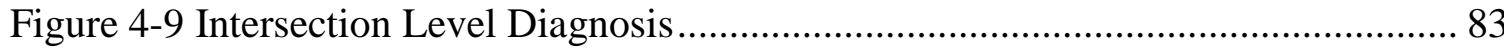

Figure 4-10 Individual Phase and Sectional Level Diagnosis Results between 3:00 PM and 3:59 PM 
Figure 5-1 Comparison of Signal Phase Diagnosis Results for EBL Movement ............ 94

Figure 5-2 Comparison of Signal Phase Diagnosis Results for NBL Movement............. 94

Figure 5-3 Comparison of Signal Phase Diagnosis Results for NBT Movement............. 95

Figure 5-4 Comparison of Signal Phase Diagnosis Results for SBL Movement ............ 95

Figure 5-5 Comparison of Signal Phase Diagnosis Results for SBT Movement ............ 96

Figure 5-6 Comparison of Signal Phase Diagnosis Results for WBL Movement............ 96

Figure 5-7 Comparison of Signal Phase Diagnosis Results for EBT Movement ............. 97

Figure 5-8 Comparison of Signal Phase Diagnosis Results for WBT Movement............ 97

Figure 5-9 Comparison of Signal Diagnosis Results for the Intersection ...................... 98 


\section{ABBREVIATIONS AND ACRONYMS}

\begin{tabular}{|c|c|}
\hline A-PeMS & Arterial Performance Measurement System \\
\hline AASHTO & American Association of State Highway and Transportation Officials \\
\hline $\mathrm{ACS}$ & Adaptive Control Software \\
\hline AVI & Automatic Vehicle Identification \\
\hline AVL & Automatic Vehicle Location \\
\hline $\mathrm{CDF}$ & Cumulative Distribution Function \\
\hline CID & Controller Interface Device \\
\hline DBSCAN & Density-Based Spatial Clustering of Applications with Noise \\
\hline DCU & Data Collection Unit \\
\hline EBL & Eastbound Left Turn \\
\hline EBT & Eastbound Thru \\
\hline EDT & Eastern Daylight Time \\
\hline EST & Eastern Standard Time \\
\hline FDOT & Florida Department of Transportation \\
\hline FHWA & Federal Highway Administration \\
\hline GOR & Green Occupancy Ratio \\
\hline INDOT & Indiana Department of Transportation \\
\hline ITS & Intelligent Transportation Systems \\
\hline KITS & Kimley-Horn Integrated Transportation System \\
\hline LPCM & Link Pivoting Combination Method \\
\hline LWR & Lighthill-Whitham-Richards shockwave theory \\
\hline
\end{tabular}




\begin{tabular}{|c|c|}
\hline MAC & Media Access Control \\
\hline MNDOT & Minnesota Department of Transportation \\
\hline MOE & Measure of Effectiveness \\
\hline MR & Max-out Ratio \\
\hline NBL & Northbound Left Turn \\
\hline NBT & Northbound Thru \\
\hline NEMA & National Electrical Manufacturers Association \\
\hline OPAC & Optimized Policies for Adaptive Control \\
\hline PCD & Purdue Coordination Diagram \\
\hline PeMS & Performance Measurement System \\
\hline PMRG & Performance Measure Report Generator \\
\hline POG & Percent of vehicle arriving On Green \\
\hline SBL & Southbound Left Turn \\
\hline SBT & Southbound Thru \\
\hline SHRP2 & Second Strategic Highway Research Program \\
\hline \multicolumn{2}{|c|}{ SMART-SIGNALSystematic Monitoring of Arterial Road Traffic and Signals } \\
\hline SPMs & Signal Performance Metrics \\
\hline SQL & Structured Query Language \\
\hline TMP & Turning Movement Proportion \\
\hline TOD & Time-of-Day \\
\hline TSER & Traffic Signal Event Recorder \\
\hline TSPMS & Traffic Signal Performance Monitoring System \\
\hline
\end{tabular}




$\begin{array}{ll}\text { TTI } & \text { Travel Time Index } \\ \text { TxDOT } & \text { Texas Department of Transportation } \\ \text { UDOT } & \text { Utah Department of Transportation } \\ \text { UTC } & \text { Coordinated Universal Time } \\ \text { V/C ratio } & \text { Volume-to-Capacity ratio } \\ \text { VHT } & \text { Vehicle Hours Traveled } \\ \text { VMT } & \text { Vehicle Miles Traveled } \\ \text { WBL } & \text { Westbound Left Turn } \\ \text { WBT } & \text { Westbound Thru }\end{array}$




\section{CHAPTER 1}

\section{INTRODUCTION}

\subsection{Research Background}

Identifying existing signal control problems is one of the most challenging tasks currently confronting transportation agencies. Traditionally, traffic signal control problems are either identified by signal operation personnel through field observations or are based on drivers' complaints. Otherwise, a signal is usually reevaluated and retimed as necessary or periodically, and the frequency of the reevaluation depends on practitioner resources and experience.

Data collections for signal timing evaluation can be expensive and labor-intensive. For instance, a basic, important input to the traditional signal timing optimization software, such as SYNCHRO and TRANSYT-7F, is turning movement counts at intersections. Collecting turning movement counts requires personnel to stay near an intersection or in their offices and watch videos and spend hours manually counting the number of vehicles for each movement. Despite the application of newer data collection aid techniques, such as video recording and automated data input devices, collecting turning movement counts still requires great effort. Consequently, in common practice, turning movement counts are only available for one to three days out of a year. These turning movement counts are normally combined with three to seven days of tube counts at several locations of the arterial in an attempt to capture the variability of demands between days. 
The collected data are typically adjusted, averaged, and aggregated into 15-minute bins, as required by modeling software packages. Although this is the most cost-effective option in many cases, it is obviously not adequate enough to predict the large variations in traffic patterns between days, and thus to account for signal timing plan selections. Developing signal timing plans based on the aggregated data from only a few days may lead to inaccurate or biased signal operation assessment of the intersection (Bullock et al. 2014). The same type of problem is also found in other manual data collection procedures for various performance measures.

With the implementation of central signal control systems, detector and signal malfunctions are automatically reported to traffic control centers. In addition, the data uploaded to the traffic control centers have traditionally included the status of stop-line detectors and signal phase data at signalized intersections. However, these data are only used in a limited fashion to support decisions associated with signal control. On the other hand, with the emergence of Intelligent Transportation Systems (ITS) detection technologies and increasing deployment of ITS devices along arterial streets, such as Automatic Vehicle Identification (AVI) technologies (e.g., Wi-Fi sensors, Bluetooth sensors, vehicle signature matching based on magnetometers), point detectors, and advanced control systems, detailed data are becoming available to support traffic control decisions. However, as stated above, such utilization is limited.

Collecting detailed traffic data from the aforementioned multiple sources for signalized arterial streets is increasingly being considered and performed by transportation agencies. However, there are limited efforts on the use of such data for better management of these streets in current traffic signal practices. Given a 69 out of 100, the 2012 National 
Traffic Signal Report Card graded the existing traffic signal operation with a "D+" letter grade (National Transportation Operations Coalition 2012). In the subcategories of traffic signal operation, the signal timing practices received a " $\mathrm{C}$ " grade, whereas traffic monitoring and data collection were rated as "F." It was reported that most agencies analyzed signal timing settings such as cycle lengths, offsets, phase sequence, and green splits as part of their traffic signal implementation and evaluation. However, in terms of traffic monitoring and data collection, only a small portion of agencies show significant effort in developing performance monitoring systems, assessing the quality of the collected data, or archiving traffic data. This implies a gap between signal timing practice (e.g., signal evaluation, optimization, etc.) and related technical support (e.g., data collection, data analysis, decision support, etc.), which is usually due to either lack of data or analysis tools.

\subsection{Problem Statement}

Without further utilization of the data despite availability, practitioners have little information about the signal operations, except for detecting malfunctions. Manual field observations could be conducted, but they are expensive and labor-intensive. Moreover, it is impossible to conduct field observations frequently for a large number of intersections. Another limitation of the current practices is the difficulty of monitoring and evaluating signal operation continuously, other than for a short period of time.

\subsection{Goal and Objectives}

The goal of this dissertation is to develop a framework for automatic decision-support signal operation diagnosis that uses a combination of relatively low-cost data from AVI 
sensors and data from existing signal controllers. The specific objectives of this dissertation are as follows:

1. Develop data preprocessing procedures to prepare valid data for use in assessing signal operation from AVI sensors and existing signal controllers, including data matching, data filtering, and data aggregation procedures.

2. Develop a diagnostic scheme to diagnose the traffic signal operations and provide recommendations for improvements, using data from AVI sensors combined with historical signal timing records from the signal controller.

\subsection{Dissertation Organization}

This dissertation is organized into six chapters. Chapter 1 introduces the background of this dissertation research, describes the problems to be solved, and sets the goals and objectives to be achieved.

Chapter 2 presents a literature review of the existing studies on signal operation diagnosis, including data collection, performance measure utilization, and signal operation evaluation. The main purpose of this review is to understand the state-of-the-art practices on signal operation diagnosis and their advantages and disadvantages.

Chapter 3 describes the methodology developed in this dissertation for the proposed framework of traffic signal operation diagnosis, which includes data acquisition and preprocessing, performance measure selection and generation, criteria establishment, and signal control problem identification. The complete three-level signal operation diagnosis scheme developed in this dissertation is described in detail herein. 
Chapter 4 details the implementation of the developed framework to assess the signal operation of a real-world signalized intersection, followed by an evaluation of the framework's performance.

Chapter 5 discusses the performance of the framework with various values from the corresponding thresholds. The impacts of the thresholds on the diagnosis results are also presented in this chapter.

Finally, Chapter 6 summarizes the findings from this dissertation, highlights the research contributions, and provides recommendations for future study. 


\section{CHAPTER 2}

\section{LITERATURE REVIEW}

\subsection{Introduction}

Limited studies and practices on traffic signal diagnosis can be found in the current literature. In order to diagnose a traffic signal operation, which is the major goal of this dissertation, various performance measures are needed. With the appropriate and adequate performance measures, it is possible to identify traffic patterns and problems associated with signal control systems. In this context, rich data are critical to assure the generation of credible performance measures for signal operation diagnosis.

\subsection{Automated Data Collection and Utilization for Traffic Signal Operation Diagnosis}

Even though automated traffic data collection, archiving, and utilization are not new concepts, most related efforts are geared toward freeway systems rather than arterial streets. An example is the freeway Performance Measurement System (PeMS) (Chen 2002), which collects real-time traffic data from point traffic detectors and estimates Vehicle Miles Traveled (VMT), Vehicle Hours Traveled (VHT), and travel times. PeMS is a web-based tool that provides reports for traffic planners, operators, and engineers.

With the emergence of a wider use of ITS detection technologies on arterial streets, such as Wi-Fi sensors, Bluetooth readers, upstream point detectors, etc., data for signalized arterial streets become richer and more complex. In combination with information from modern signal controllers and additional stop-line detectors, a full picture of the operation at signalized intersections can be delineated. Several studies investigated the subject of collecting and analyzing data to extract performance measures for traffic signals. 


\subsubsection{Automated Data Collection and Performance Measure Generation}

The Arterial Performance Measurement System, or A-PeMS, was proposed to adopt similar concepts to those for freeway performance measures, as the aforementioned PeMS. It is also a web-based system that was developed to automate processes for data collection and processing on arterial streets (Petty and Barkley 2011). It utilizes various types of sensor information, such as flows, speeds, travel times, and so on, combined with signal timing information, including static plans and real-time measurements, and applies it to the signalized arterial streets. A-PeMS was designed to process multiple types of sensor data, such as simple loops data, video-based detection, Bluetooth tag readings, data from automatic vehicle identification technology, etc. The availability of those sensor data is location-based.

A-PeMS computes and graphs the travel time distribution, flow rates, densities, and average speeds during the user-selected month and hour of the day. With the installed Sensys ${ }^{\circledR}$ wireless vehicle detectors, which applies an AVI technology (also known as vehicle re-identification technology), the A-PeMS is able to visualize corridor performance by producing time-space coordination diagrams generated for coordinated traffic signal control systems. This assessment is based on the Purdue Coordination Diagram (PCD), which was previously produced (Day et al. 2009), and requires high-resolution signal event data. The high-resolution signal event data collect all phase and detector status changes, such as signal indication changes, detector actuation on and off records, and so on, at a resolution of 0.1 seconds. The PCD shows the percentage of vehicles arriving on green at an intersection, allowing the assessment of signal progression. 
In addition, A-PeMS can calculate turning movement counts if the data are sufficient. A-PeMS also collects the status of the detectors and thus can access the detector health and identify the non-working detectors and the reasons for the malfunction. It is also worth noting that as part of the A-PeMS system, an analytical model was proposed to estimate the travel time on arterial streets with loop detector data and signal settings (Skabardonis and Geroliminis 2008).

Another attempt to automate data collection on arterial streets is the development of the Traffic Signal Performance Monitoring System (TSPMS) (Balke et al. 2005). TSPMS was developed to assist the Texas Department of Transportation (TxDOT) with automatically collecting or generating performance measures, including the following:

- cycle time

- time to service

- queue service time

- duration of the green time

- yellow, all-red and red interval for each phase

- number of vehicles entering the intersection during each interval

- yellow and all-red violation rates

- phase failure rate

TSPMS uses a Traffic Controller Interface Device (CID) to receive electric signals from the traffic signal control system, which is, in this case, the Eagle ${ }^{\circledR}$ EPAC 300 controller. The CID is a type of hardware that has a physical connection between the signal controller and a computer inside the cabinet on site. The Traffic Signal Event Recorder (TSER), which is a software program, is set up in TSPMS to record the status changes of 
various outputs from the traffic signal controller and the traffic detector according to the electric signals received by the CID. The TSER also stores the time that the changes occurred in the daily log files. Finally, another software program, called the Performance Measure Report Generator (PMRG), is developed to analyze the log files and calculate the mentioned performance measures based on the daily log files created by the TSER, which stores the changes in status, as well as the time the change occurred.

The researchers applied TSPMS to two different intersections in order to assess its ability to collect the performance measures. All results from the TSPMS are based on the data collected from loop detectors, and thus the system's effectiveness and accuracy highly rely on the design of the detection system and the placement of the detectors.

With a similar architecture to the one employed in TSPMS, a system for highresolution event data collection, named Systematic Monitoring of Arterial Road Traffic and Signals (SMART-SIGNAL), was proposed for data collection and performance monitoring for closed-loop signal control systems (Ma 2008; Liu et al. 2008). Historical traffic signal control data, including all actuation records on detectors and phase changes on traffic signals, can be archived and stored by SMART-SIGNAL. The system was installed in 2007 at eleven intersections along an arterial corridor in Minnesota for automatic data collection.

In addition to the high-resolution traffic signal event data collection, SMARTSIGNAL also generates arterial performance measures in real time. Algorithms were developed for queue length and turning movement proportion (TMP) estimations by combining mathematical models and the high-resolution event data collected by the system. An innovative algorithm was also proposed to extract performance measures by tracing 
virtual probe vehicles, of which the maneuver decisions depended on a set of traffic state variables. Three possible maneuvers, acceleration, deceleration, and no-speed-change, can be predicted using the decision-tree technique. The aggregated statuses of the virtual probe vehicles are used to estimate time-dependent arterial travel times, as well as other performance measures, such as delay and number of stops. Additionally, with the highresolution event data collected by SMART-SIGNAL, a data-intensive time-dependent arterial travel time estimation model was developed in another study based on the aggregation of intersection delays using the times of arrival (Liu and Ma 2007).

Other than TSPMS and SMART-SIGNAL, more efforts can be found on collecting and utilizing high-resolution event data that record and store all status changes of detectors and signal indications. The Federal Highway Administration (FHWA) conducted a research program on ACS-Lite, a reduced-scale version of Adaptive Control Software (ACS). One of the achievements of the program is the enhancement of traffic controllers from multiple vendors so that not only the timestamps when signal phase changes but also the timestamps when the detector state changes (i.e., actuation on and actuation off) were able to be recorded and logged. As a result, the high-resolution event data are collected by more traffic signal controllers, compared to the previous studies that only accessed a few.

A similar attempt was made by the American Association of State Highway and Transportation Officials (AASHTO), in cooperation with the Utah Department of Transportation (UDOT), the Indiana Department of Transportation (INDOT), and the Minnesota Department of Transportation (MNDOT), to develop an automated traffic Signal Performance Metrics (SPMs) program that shows the real-time and historical performance measures at signalized intersections (AASHTO 2014). In contrast to the 
TSPMS and SMART-SIGNAL system mentioned previously, which collects data locally, the SPMs program automates data collection by deploying a communication architecture that can transmit high-resolution event data to the traffic management center. The metrics are generated from high-resolution event data and implemented in a web interface, along with the following performance measures:

- approach delay

- approach volume

- arrivals on red

- Purdue coordination diagram

- Purdue phase termination

- $\quad$ speed

- $\quad$ split monitor

- turning movement counts

\subsubsection{Automated Signal Diagnosis Attempts}

The SPMs online tool can identify detector malfunctions, vehicle delays, speeds, and travel times, as well as evaluate the quality of signal control and progression of traffic. The development of SPMs is based on several previous studies, which were conducted to investigate new performance measures that could depict flow rates, quality of coordination, and split failures, with three traffic signal controller vendors, Econolite ${ }^{\circledR}$, Siemens ${ }^{\circledR}$, and Peek® (Smaglik et al. 2007; Li et al. 2013; Day et al. 2009).

One of the studies that contributed to the development of the SPMs attempted to automate the data collection by utilizing the National Electrical Manufacturers Association 
(NEMA) controller with an enhanced Econolite ${ }^{\circledR}$ ASC/3 controller software. The timestamped detector and phase state changes are collected (Smaglik et al. 2007). These eventbased data are collected cycle-by-cycle and utilized to calculate equivalent hourly volume, arrival-types, delays, and volume-to-capacity ratio (v/c ratio). The study demonstrated the capability of high-resolution event data in generating quantitative graphs for the purpose of assessing progression and intersection delay.

Another research group used a system engineering approach to identify three objectives of signal operation, including reliable communication of signal systems, good allocation of green times, and good progression ( $\mathrm{Li}$ et al. 2013). As part of that research, the authors proposed a method to identify the potential opportunities for reallocating the green time by analyzing the information provided regarding phase force-offs (i.e., maxouts) and gap-outs.

The authors suggested that sustained demands can be identified for a phase if it has max-outs (reaches the maximum green) in three or more consecutive cycles. The researchers studied the typical dual-ring eight-phase signal control, as shown in Figure 2-1, and defined pairs of companion phases. Each of the companion phases in a pair conflicted with the other phase in the same ring, and thus four pairs of companion phases were defined: $\{1,2\},\{3,4\},\{5,6\}$, and $\{7,8\}$. According to the study, if a phase encountered max-outs during three or more consecutive cycles, and gap-outs were always found in its companion phase during those cycles, these cycles were then marked as opportunities for potential split changes.

The percentages of cycles marked as opportunities for potential split adjustment are calculated for a time-of-day (TOD) plan. The authors only considered the cases when the 
aforementioned percentages were higher than an arbitrary 5\% threshold as the candidates for split adjustments. They also ranked the opportunities for split changes throughout the entire system, with hundreds of traffic signals to prioritize the needs.

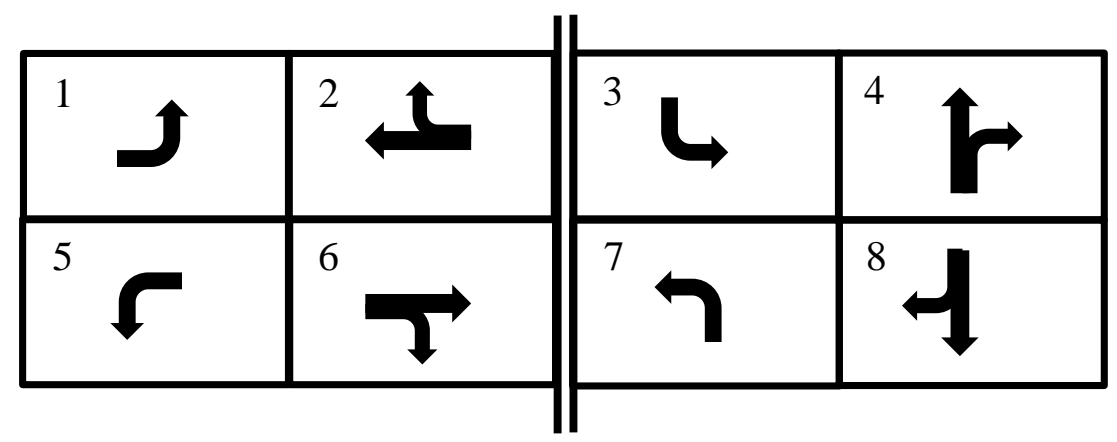

Figure 2-1: Typical Dual-Ring Eight-Phase Diagram

The quality of progression in coordinated traffic signal systems is assessed using the PCD. The PCD can be constructed from the high-resolution event data that records every vehicle's arrival time at the intersection (Day et al. 2009). The arrival pattern, such as the platoon arrival on green, platoon arrival on red, random arrivals, etc., can be visually represented in the PCD. With the high-resolution event data, the percent of vehicles arriving on green (POG) is calculated simply by dividing the total number of vehicles arriving on green by the approach volume. The vehicle arrival pattern and the associated POG can be predicted by shifting the PCD with different time offsets. The study found that it is able to improve the offset by finding the maximum value of the total number of vehicles arriving on green in concurrent phases.

An analogous study was conducted later to develop and assess procedures that optimize the offset by aggregating the high-resolution event data (Day and Bullock 2010). The study found that a Link Pivoting Combination Method (LPCM) algorithm performs the best among other tested algorithms, such as the hierarchical heuristic search, Monte 
Carlo simulation, genetic algorithms, and TRANSYT-style hill climbing, in coordinated signal offset optimization.

Smaglik and his co-authors introduced the Green Occupancy Ratio (GOR) as a measure, which is used to quantify the operation of traffic signals, and compared it to the traditional v/c ratio metric (Smaglik et al. 2011). The GOR is calculated as the stop bar detector occupancy divided by the duration time of the corresponding green phase. The v/c ratio, as the name suggests, is the quotient of volume divided by the capacity. The $\mathrm{v} / \mathrm{c}$ ratio was found to be a more robust performance measure than the GOR, which is sensitive to detection zone lengths and vehicle speeds. However, the GOR was still suggested by the authors to be a reasonable surrogate in the real-time performance assessment of traffic signal systems due to its easy accessibility. In another study, arrival types were generated to assess the performance of a coordinated traffic signal system by collecting phase status and time-stamped vehicle arrivals (Smaglik et al. 2007). Nonetheless, several issues were noted with this assessment of arrival types, such as queues beyond the setback detectors, appropriateness of the moving averages, and so on.

Zheng and other researchers developed a flexible and low-cost data collection unit (DCU) to enhance the previously described SMART-SIGNAL system on high-resolution event data collection. The offset, green split, and cycle length can be extracted from the collected data (Zheng et al. 2013). The high-resolution event data collected by the proposed DCU is similar to the data mentioned earlier and includes detector events, such as occupation start time (i.e., actuation-on time), duration length (such that the actuation-off time can be derived), detector ID, and signal events with signal start time, duration time, signal status, and phase ID. 
The authors proposed a diagnosis tool to simultaneously diagnose offset, green split, and cycle length. The offset diagnosis in the proposed tool is based on the aforementioned LPCM algorithm, while the green split diagnosis relies on the GOR introduced by Smaglik et al. The cycle length diagnosis depends on the combination of two types of evaluations: 1) intersection capacity evaluation based on Webster's optimal cycle length formula; and 2) coordination evaluation based on MAXBAND's maximized bandwidth. In another parallel study, the authors added a module of TOD transition optimization and fine-tuning based on a mathematical total delay minimization (Liu and Zheng 2014).

More pilot studies of utilizing high-resolution event data to diagnose signal operation can be found in literature. Two techniques, the input-output technique and a hybrid technique, were proposed to estimate approach delays and maximum queue lengths in real-time (Sharma et al. 2007). The input-output technique utilizes setback detectors to provide real-time arrival profiles. Moreover, it generates the departure profiles using models of traffic flow characteristics, including start-up lost time, storage capacity, saturation headway, queue discharge headway, and the signal phase change data. On the other hand, both profiles of arrivals and departures can be extracted by the hybrid technique in real-time, which employs data from setback detectors, stop bar detectors, and signal controller.

Using high-resolution event data, another researcher group estimated timedependent queue length by identifying traffic state changes with the Lighthill-WhithamRichards (LWR) shockwave theory (Liu et al. 2009). The proposed queue length estimation algorithm is proven to produce good results, even under congested conditions. Queue 
lengths are usually difficult to estimate in traffic congestion, since the back of queue is often at the upstream of the setback detectors.

\subsection{Summary}

As is evident from the current literature discussed above, efforts have been limited on automated data collection and utilization on signalized arterial streets, as well as on automated signal diagnosis. Although relatively recent research activities are able to produce significant advancements on the topic, they usually require unavailable detector and controller data. In particular, the majority of previous studies on the related subject rely on high-resolution event data, which are not readily available from existing signal control systems. For example, Zheng and the research team developed a complete system for signal diagnosis; however, it still requires high-resolution event data (Zheng et al. 2013).

Albeit the fact that high-resolution event data can provide detailed and accurate information on signal operations, collecting this type of data requires hardware and software updates that are not always feasible for existing intersection control systems. In addition, in the current practices, the lane groups associated with the coordinated phases usually do not have detectors installed, which is required in the methods developed based on high-resolution event data collection. Therefore, prevailing signal control systems are still not equipped to collect the high-resolution event data required by the recently developed methods. Thus, there is a need for alternatives including developing methods, which allow diagnosing of the signal operation based on lower cost data.

On the other hand, it has been proven that multiple performance measures can be extracted from high-resolution event data in the previous efforts. A more detailed review 
on high-resolution event data can be found in an overview paper (Wu and Liu 2014). Nevertheless, most of the previous studies still rely on human judgement for signal diagnoses. For example, the TSPMS and SMART-SIGNAL approaches relate signal timings to traffic conditions by aggregating both signal events and detection records. However, they only calculated and reported the performance measures, and did not provide method for diagnosing signal operation. In other words, they only provided information and no recommendations on improving signal operations. Similarly, the PCD approach provides excellent visualization of arrival patterns and can assist practitioners in evaluating the traffic signal coordination plans, but no automated method is offered to provide recommendations regarding signal operations.

A number of studies can be found on travel time and travel speed estimation without high-resolution event data (Frechette and Khan 1998; Courage et al. 1998; Turner et al. 1998; Zhang 1999; Cheu et al. 2001; Dailey and Cathey 2002). However, these studies do not support the purpose of signal operation diagnosis, specifically the type addressed in this dissertation.

As one of the few attempts to automate data collection and utilize it for signalized arterial streets, A-PeMS demonstrates the feasibility of aggregating data from multiple sources with or without high-resolution event data to reflect the traffic conditions on arterial streets. Nevertheless, it focuses on the arterial links with multiple intersections rather than an individual intersection. At the intersection level, A-PeMS only reports the signal timings; it does not apply the data to the investigation. In other words, A-PeMS does not relate the signal timing to the traffic conditions, and thus cannot assess signal operation, except for the quality of coordination. 
It is also noteworthy that a part of the study conducted by Li and the research team (2013) provides a concept on diagnosing green split allocation to some extent without highresolution event data, but the utilized method is only based on historical signal status records (Li et al. 2013). Such diagnosis may be biased since analyzing signal records alone without information on traffic conditions is not sufficient. For example, consecutive maxouts of a phase may be the result of intersection blockage by its downstream traffic instead of sustained, high levels of demands as suggested by the study. Another drawback of the diagnosis proposed by Li et al. (2013) is that it does not evaluate the opportunity of green time adjustment between the two signal phase groups located on the two sides of the dualring barrier, i.e., the potential adjustment between phase $\{1,2,5,6\}$ and $\{3,4,7,8\}$, as shown in Figure 2-1. In addition, that study only focuses on diagnosing green time allocation (i.e., green time adjustment as quoted in their paper), and thus it is not a holistic signal operation diagnosis. 


\section{CHAPTER 3}

\section{METHODOLOGY}

\subsection{Introduction}

As can be concluded from the literature review, there are no existing adequate methods to automate traffic signal operation diagnosis, particularly without high-resolution event data. To better assist decision-makers in traffic signal operation evaluation and traffic signal retiming processes with prevailing traffic signal controllers that do not allow an easy collection of high-resolution data, this dissertation proposes a framework of automatic decision-support traffic signal operation diagnosis that utilizes a combination of existing relatively low-cost archived data from Wi-Fi sensors combined with readily available (nonhigh-resolution) archived data from existing traffic signal controllers.

Figure 3-1 presents the framework of the developed methodology for this dissertation, which includes data acquisition, data preprocessing, signal operation diagnosis, and diagnosis assessment. In this chapter, the utilized data is introduced, followed by a detailed description of data preprocessing procedures for $\mathrm{Wi}-\mathrm{Fi}$ data and signal data. Multiple data preprocessing procedures are applied to Wi-Fi data, including data matching, data filtering, and data aggregation. Next, a methodology of combining different sources of data (i.e., Wi-Fi data and signal data) to evaluate the current signal operation and provide recommendations for improvements is demonstrated. Finally, the diagnosis results for the traffic signal operation are assessed by comparing them with the field observations. 


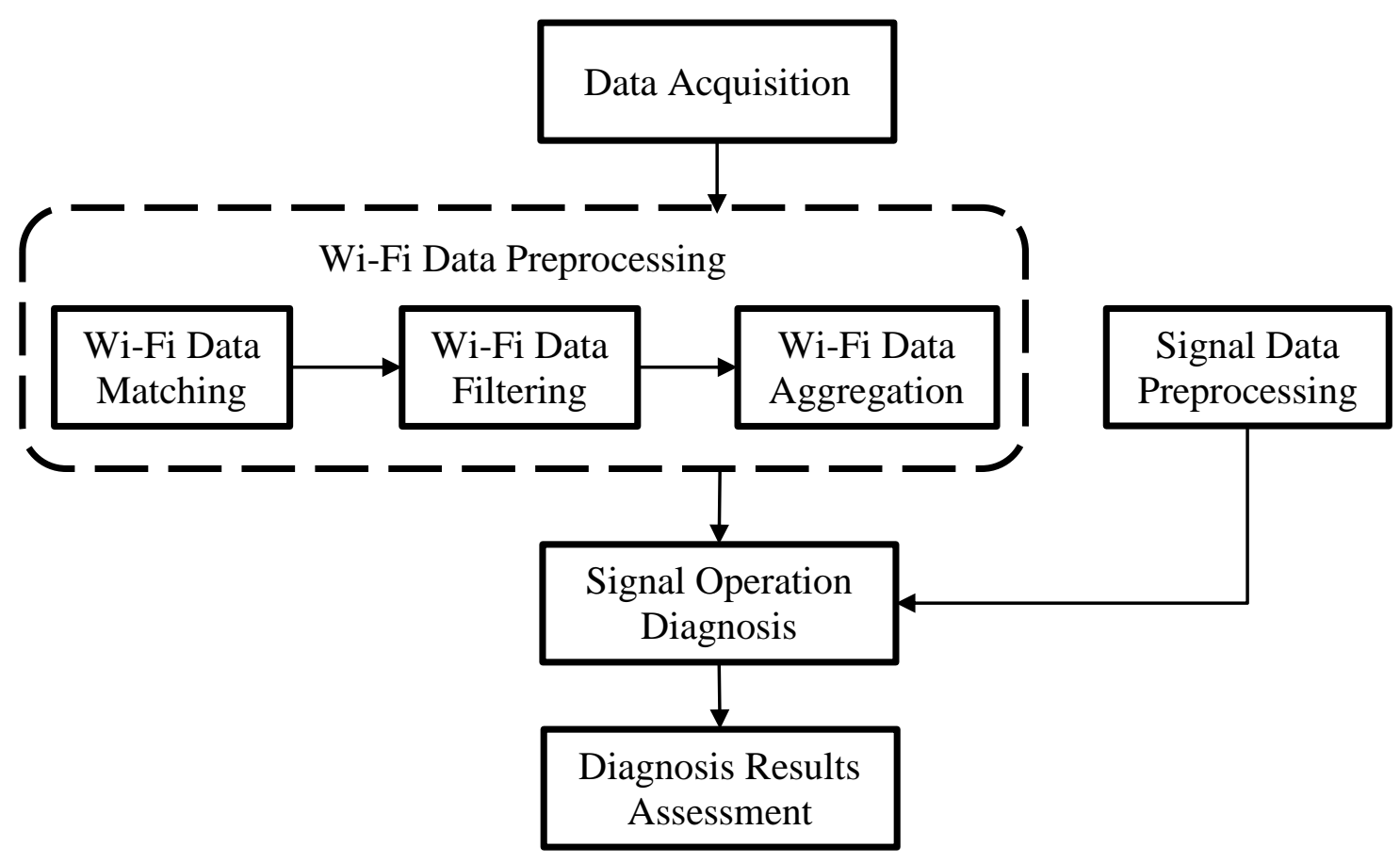

Figure 3-1: Methodology Framework

\subsection{Data Acquisition}

In order to monitor the vehicular traffic conditions at a signalized intersection, data sources other than signal phase status are needed. Many technological advances can be found in data collection nowadays, such as traffic point detectors (e.g., loop detectors, video image detectors, optical detectors, ultrasonic detectors, microwave sensors, magnetometers, etc.), automatic vehicle identification (AVI) systems (e.g., electronic toll collection, license plate readers, signature re-identification based on magnetic sensors, Wi-Fi sensors, Bluetooth sensors, etc.), automatic vehicle location (AVL) systems (e.g., global positioning systems, cellular communication systems, etc.), and emerging connected vehicles. Each technology has its strengths and weaknesses, and the appropriate selection depends on many aspects, for example, installation costs, maintenance costs, type and quality of the collected data, endurance, and so on. The comparison and selection of the data collection means are 
beyond the scope of this dissertation, and thus are not explained here in detail. The developed methodology in this dissertation uses the Acyclica ${ }^{\circledR} \mathrm{Wi}$-Fi sensors, but any AVI sensor can be utilized, such as Bluetooth, magnetometer-based, and license-plate readers. The reason for selecting the AVI sensors (i.e., Wi-Fi sensors in this case) to develop the methodology of traffic signal operation diagnosis is because this type of AVI devices are low-cost for both installation and maintenance, are easily installed, and produce the required input data.

The Wi-Fi sensors were fit inside the signal controller cabinets, as shown in Figure 3-2. A Wi-Fi sensor records the Wi-Fi Media Access Control (MAC) addresses of all the devices within its detection range radius, along with the associated reading time. Devices such as cell phones, laptops, etc., that have Wi-Fi are continuously detected as long as they are within the sensor's detection range. The strength of the received Wi-Fi signals is also recorded by the sensor. The signal strengths increase with the decrease of the distance between the detected devices and Wi-Fi sensor.

It is worth mentioning that the Acyclica ${ }^{\circledR} \mathrm{Wi}-\mathrm{Fi}$ sensors adopt the UNIX time (or Epoch time in some references). UNIX time counts the number of seconds elapsed since 0:00:00 AM on January 1, 1970, in the Coordinated Universal Time (UTC) time standard format. Thus, the precision of the Wi-Fi readings is one second of time. The human readable times extracted from the Wi-Fi sensors herein are converted from UNIX time to Eastern Standard Time (EST) or Eastern Daylight Time (EDT), depending on the time of year of the data collection.

With the application of data matching and filtering algorithms that will be discussed later, each vehicle with an activated Wi-Fi device can be identified at different locations 
relative to an intersection based on the uniqueness of the MAC address, the time detected, and the signal strength. An example of the raw data from a Wi-Fi sensor is shown in Table 3-1. The serial column in the table displays the sensor identification number (sensor ID). The sensor ID is unique for each Wi-Fi sensor and thus can be used to identify intersections or locations where the sensors are installed. The Wi-Fi sensor assigns an encrypted MAC address to each identified device and logs every detection with the received signal strength, as well as the timestamp when the signal is detected. Four stages of Wi-Fi detection can be identified:

1. The first detection: the timestamp when the device is first detected

2. The last detection: the timestamp when the device is last detected

3. The maximum strength detection: the timestamp when the detection strength first reaches the maximum value

4. Other detection: all timestamps other than the above three

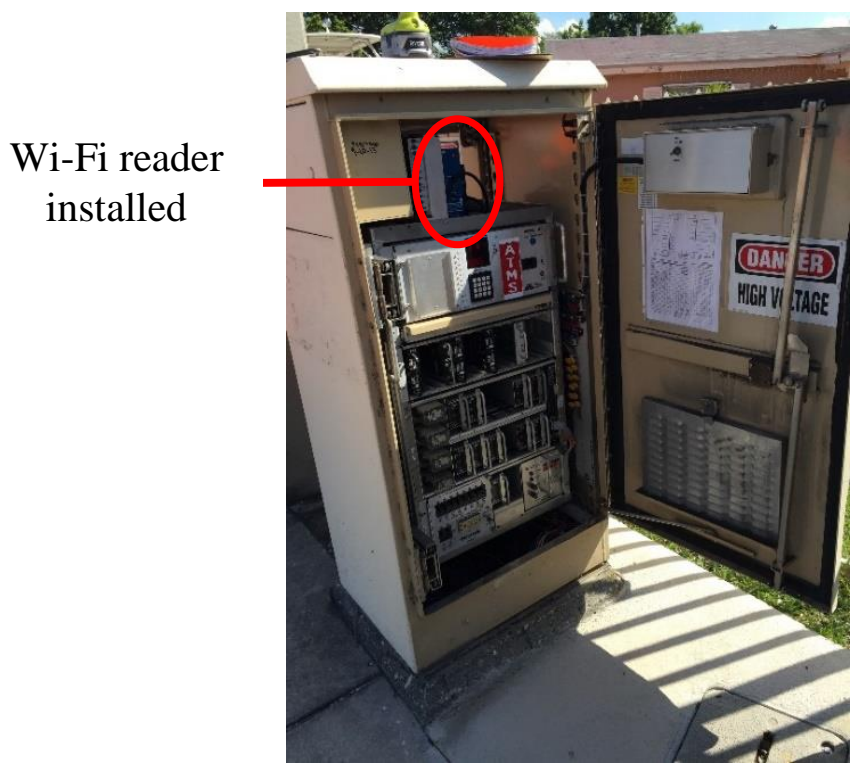

Figure 3-2 Wi-Fi Sensors Installed inside the Traffic Signal Controller Cabinet 
Due to the space limit, not all detection records of the example device are shown in Table 3-1. Using the example in Table 3-1, the first detection of the device by the Wi-Fi sensor " 265276 " is at the timestamp of " 1449192285 ," or on Thursday, December 3, 2015 at 8:24:45 PM EST, since it is the first time the device was detected by the sensor. The maximum strength detection of the device occurs at the timestamp of "1449192311" (on Thursday, December 3, 2015 at 8:25:11 PM EST), as the signal strength first reached the maximum value of -54 . The last detection of the device is recorded at the timestamp of “1449192312” (on Thursday, December 3, 2015 at 8:25:12 PM EST). The remaining records are labeled as other detections. A combination of those four detection stages can be applied for different purposes. Details will be given in a later section.

Table 3-1 Wi-Fi Detection Raw Data Example

\begin{tabular}{|c|c|c|c|}
\hline Timestamp & MAC Address & Strength & Serial \\
\hline 1449192285 & $\begin{array}{c}\text { 000812c00ec133d56e0fe87cb83847f8512e464 } \\
\text { 8e38c5a458b7bd91ee9b2b976 }\end{array}$ & -61 & 265276 \\
\hline 1449192289 & $\begin{array}{c}\text { 000812c00ec133d56e0fe87cb83847f8512e464 } \\
\text { 8e38c5a458b7bd91ee9b2b976 }\end{array}$ & -58 & 265276 \\
\hline 1449192289 & $\begin{array}{c}\text { 000812c00ec133d56e0fe87cb83847f8512e464 } \\
\text { 8e38c5a458b7bd91ee9b2b976 }\end{array}$ & -58 & 265276 \\
\hline 1449192293 & $\begin{array}{c}\text { 000812c00ec133d56e0fe87cb83847f8512e464 } \\
\text { 8e38c5a458b7bd91ee9b2b976 }\end{array}$ & -58 & 265276 \\
\hline 1449192293 & $\begin{array}{c}\text { 000812c00ec133d56e0fe87cb83847f8512e464 } \\
\text { 8e38c5a458b7bd91ee9b2b976 }\end{array}$ & -61 & 265276 \\
\hline 1449192296 & $\begin{array}{c}\text { 000812c00ec133d56e0fe87cb83847f8512e464 } \\
\text { 8e38c5a458b7bd91ee9b2b976 }\end{array}$ & -60 & 265276 \\
\hline$\ldots \ldots$ & 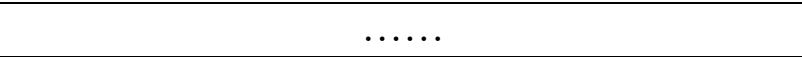 & $\ldots \ldots$ & \\
\hline 1449192311 & $\begin{array}{c}\text { 000812c00ec133d56e0fe87cb83847f8512e464 } \\
\text { 8e38c5a458b7bd91ee9b2b976 }\end{array}$ & -54 & 265276 \\
\hline 1449192311 & $\begin{array}{c}\text { 000812c00ec133d56e0fe87cb83847f8512e464 } \\
\text { 8e38c5a458b7bd91ee9b2b976 }\end{array}$ & -61 & 265276 \\
\hline 1449192312 & $\begin{array}{c}\text { 000812c00ec133d56e0fe87cb83847f8512e464 } \\
\text { 8e38c5a458b7bd91ee9b2b976 }\end{array}$ & -61 & 265276 \\
\hline 1449192312 & $\begin{array}{c}\text { 000812c00ec133d56e0fe87cb83847f8512e464 } \\
\text { 8e38c5a458b7bd91ee9b2b976 }\end{array}$ & -60 & 265276 \\
\hline
\end{tabular}


In this dissertation, the intersection of Southwest 8th Street and Southwest 107th Avenue in Miami, Florida was selected as the studied intersection for the initial development and assessment of the proposed framework. Five Wi-Fi sensors were installed at that intersection and its four adjacent signalized intersections:

1. Intersection of Southwest 8th Street and Southwest 109th Avenue

2. Intersection of Southwest 8th Street and Southwest 102th Avenue

3. Intersection of Southwest 4th Street and Southwest 107th Avenue

4. Intersection of Southwest 1100th Block and Southwest 107th Avenue

An illustration of the geographical location of the studied area is shown in Figure 3-3.

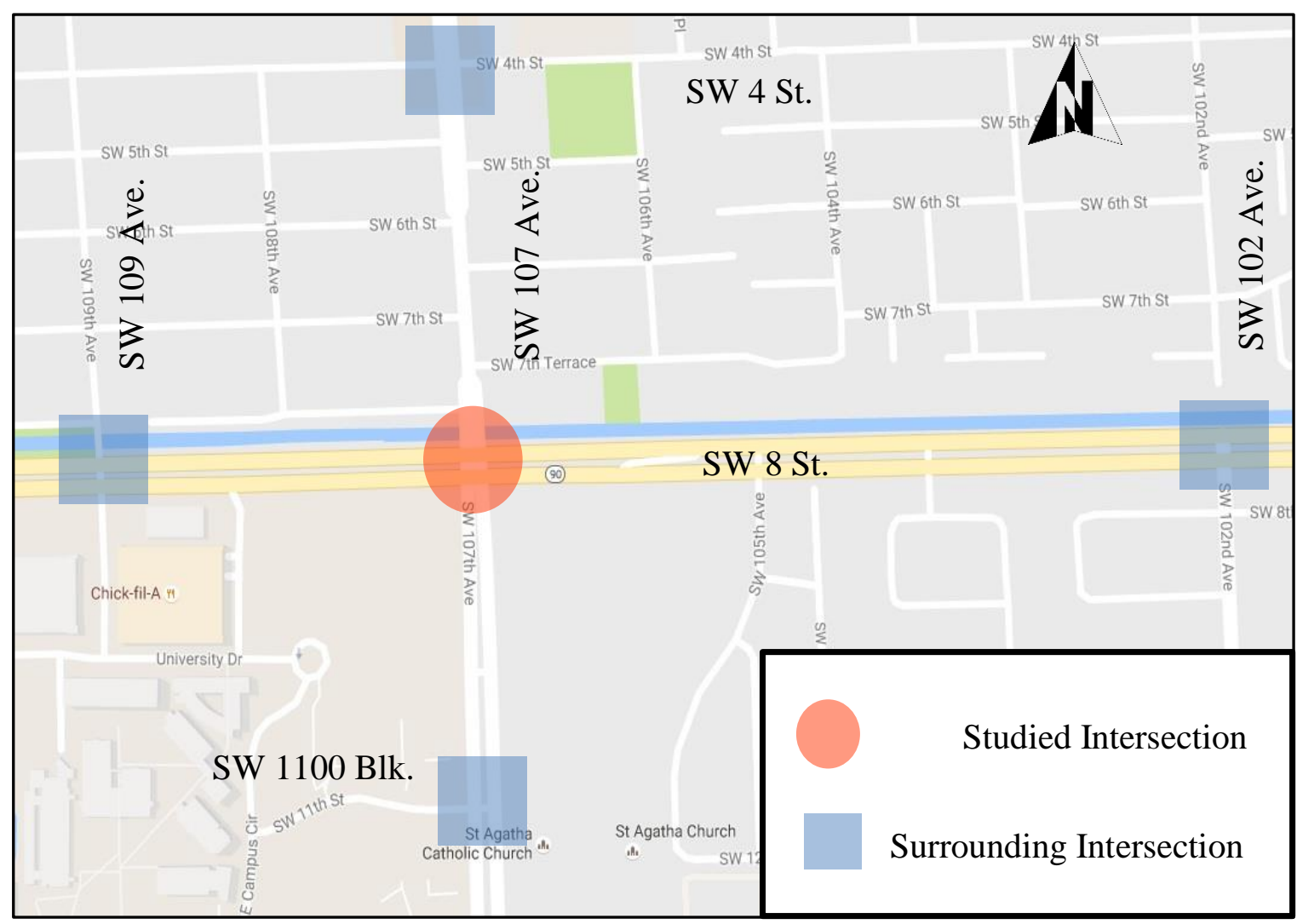

Note: Figure not drawn to scale.

Figure 3-3 Wi-Fi Detector Locations (Background image source: Map data @ 2016 Google) 
In addition to the data from $\mathrm{Wi}-\mathrm{Fi}$ sensors, historical intersection timing data are downloaded from the Kimley-Horn Integrated Transportation System (KITS®), which is the system used for signal controls in Miami-Dade County. Although the data are not in high resolution and no detector data are available, the times at which signal indications changed are logged in the archived reports. An example of the historical intersection timing data is shown in Figure 3-4.

\begin{tabular}{|c|c|c|c|c|c|c|c|c|c|}
\hline \multicolumn{2}{|c|}{$\begin{array}{l}\text { Start Time: } 12 / 17 / 15 \text { 00:00 } \\
\text { Print Date N1/15/2016 }\end{array}$} & \multicolumn{6}{|c|}{ End Time: 1/15/16 11:00 } & \multicolumn{2}{|c|}{ Print Time $\quad 4: \cap 0$ PM } \\
\hline$\underline{\text { Time }}$ & $\underline{\text { Plan }}$ & Ring 1 Phase & Interval & Grn Dur & Ring 2 Phase & Interval & Grn Dur & $\underline{\text { Status }}$ & Poll Freq \\
\hline $12 / 18 \quad 12: 27: 25$ & & $1-\mathrm{EBL}$ & & & $5-$ WBL & $\overline{\text { Clear }}$ & & & \\
\hline $12 / 18 \quad 12: 27: 31$ & & $1-\mathrm{EBL}$ & Clear & & & & & & \\
\hline $12 / 18 \quad 12: 27: 32$ & & & & & $6-\mathrm{EBT}$ & Green & 45 & & \\
\hline $12 / 18 \quad 12: 27: 38$ & & 2-WBT & Green & 39 & $6-\mathrm{EBT}$ & & & & \\
\hline $12 / 18 \quad 12: 28: 17$ & & $2-W B T$ & Clear & & $6-\mathrm{EBT}$ & Clear & & & \\
\hline $12 / 18 \quad 12: 28: 23$ & & $3-S B L$ & Green & 12 & $7-\mathrm{NBL}$ & Green & 12 & & \\
\hline $12 / 18 \quad 12: 28: 35$ & & $3-S B L$ & Clear & & $7-\mathrm{NBL}$ & clear & & & \\
\hline $12 / 18 \quad 12: 28: 41$ & & $4-\mathrm{NBT}$ & Green & 56 & & & & & \\
\hline $12 / 18 \quad 12: 28: 42$ & & $4-\mathrm{NBT}$ & & & $8-\mathrm{SBT}$ & Green & 55 & & \\
\hline $12 / 18 \quad 12: 29: 37$ & 7 & $4-\mathrm{NBT}$ & Clear & & $8-\mathrm{SBT}$ & Clear & & Coord & \\
\hline
\end{tabular}

Figure 3-4 Example of KITS® Historical Intersection Timing Report

\subsection{Wi-Fi Data Preprocessing}

Due to the nature of the detection technology, vehicles and any Wi-Fi enabled devices within the detection range of the sensors are detected. In order to obtain the travel time information for vehicles only, in particular for those traveling through the studied area without stopping for other purposes (i.e., gas, shopping, restaurant, etc.), data preprocessing methods need to be applied to the original readings. In addition, information needs to be extracted from the validated data, for example, performance measures, signal characteristics, etc. Therefore, in order to obtain useful information for later use by the signal operation diagnosis, the data preprocessing methods, including data matching, data filtering, and data aggregation, are applied to the raw data. 


\subsubsection{Data Matching}

As mentioned earlier, readings from Wi-Fi sensors consist of various types of devices. Vehicles traveling through the studied area (i.e., traversals) are the data needed to diagnose the signal operation. Travel times can be used to distinguish devices with traversals from devices with other travel modes, since traversals have different travel times in comparison with other devices, such as devices held by pedestrians, devices held by shoppers, etc. In order to obtain travel times and then extract traversals from the original readings, trips between different intersections need to be extracted from the raw readings in the format shown in Table 3-1. A trip herein is defined as a Wi-Fi enabled device that travels from one location to another.

Since a Wi-Fi device can pass an intersection multiple times, using the original readings to extract trips will omit multiple trips made with the same device. An example is presented in Table 3-2, in which two passages can be clearly identified for the same MAC address. The first passage started at the timestamp of "1449154808" (on Thursday, December 3, 2015, at 10:00:08 AM EST), and ended at the timestamp of "1449154866" (on Thursday, December 3, 2015, at 10:01:06 AM EST). The second passage started at the timestamp of “1449192285” (on Thursday, December 3, 2015, at 8:24:45 PM EST), and ended at “1449192312” (on Thursday, December 3, 2015, at 8:25:12 PM EST). As a result, the following rule is introduced for the original readings from each sensor to identify different passages made by the same MAC addresses before matching the data.

Rule 1: (Identification of Passages)

Let $p_{\alpha, j}$ be the $j^{t h}$ passage of intersection $\alpha$ made by a MAC address, a next passage $p_{\alpha, j+1}$ is identified from the previous passage $p_{\alpha, j}$ if: 


$$
t_{\alpha, i+1}-t_{\alpha, i}>60
$$

where

$t_{\alpha, i}=$ the $i^{\text {th }}$ timestamp of the MAC address from the sensor installed at intersection $\alpha$.

In other words, if two consecutive timestamps of a MAC address are more than 60 seconds apart, two different passages of an intersection made by the same MAC address are identified instead of one. This avoids the omission of multiple trips made by the same device in later matching. Furthermore, it prevents the estimation of excessive travel time by mistakenly combining multiple trips made by the same vehicle.

\section{Table 3-2 Example of Multiple Trips Made by One Device}

\begin{tabular}{|c|c|c|c|c|}
\hline Timestamp & MAC Address & Strength & Serial & Comments \\
\hline 1449154808 & $\begin{array}{c}\text { 000812c00ec133d56e0fe8 } \\
\text { 7cb83847f8512e4648e38c } \\
\text { 5a458b7bd91ee9b2b976 }\end{array}$ & -66 & 265276 & $\begin{array}{l}\text { First detection of the } \\
\text { first passage }\end{array}$ \\
\hline ..... & & ....... & ..... & \\
\hline 1449154866 & $\begin{array}{c}000812 \mathrm{c} 00 \mathrm{ec} 133 \mathrm{~d} 56 \mathrm{e} 0 \mathrm{fe} 8 \\
\text { 7cb83847f8512e4648e38c } \\
\text { 5a458b7bd91ee9b2b976 }\end{array}$ & -69 & 265276 & $\begin{array}{l}\text { Last detection of the } \\
\text { first passage }\end{array}$ \\
\hline 1449192285 & $\begin{array}{c}\text { 000812c00ec133d56e0fe8 } \\
\text { 7cb83847f8512e4648e38c } \\
\text { 5a458b7bd91ee9b2b976 }\end{array}$ & -61 & 265276 & $\begin{array}{l}\text { First detection of the } \\
\text { second passage }\end{array}$ \\
\hline$\ldots \ldots$ & ....... & ....... & $\ldots \ldots$ & $\ldots \ldots$ \\
\hline 1449192312 & $\begin{array}{c}\text { 000812c00ec133d56e0fe8 } \\
\text { 7cb83847f8512e4648e38c } \\
\text { 5a458b7bd91ee9b2b976 }\end{array}$ & -67 & 265276 & $\begin{array}{c}\text { Last detection of the } \\
\text { second passage }\end{array}$ \\
\hline
\end{tabular}

With the identified intersection passages from each Wi-Fi sensor, trips between the studied intersection and its four surrounding intersections can be extracted by matching data between the corresponding Wi-Fi sensors. In total, eight sets of matches are identified and shown in Figure 3-5. Due to the uniqueness of the MAC address of each device, one trip between a pair of intersections can be identified, if a MAC address from one Wi-Fi 
sensor is equal to the MAC address from the other sensor in the corresponding matching set.

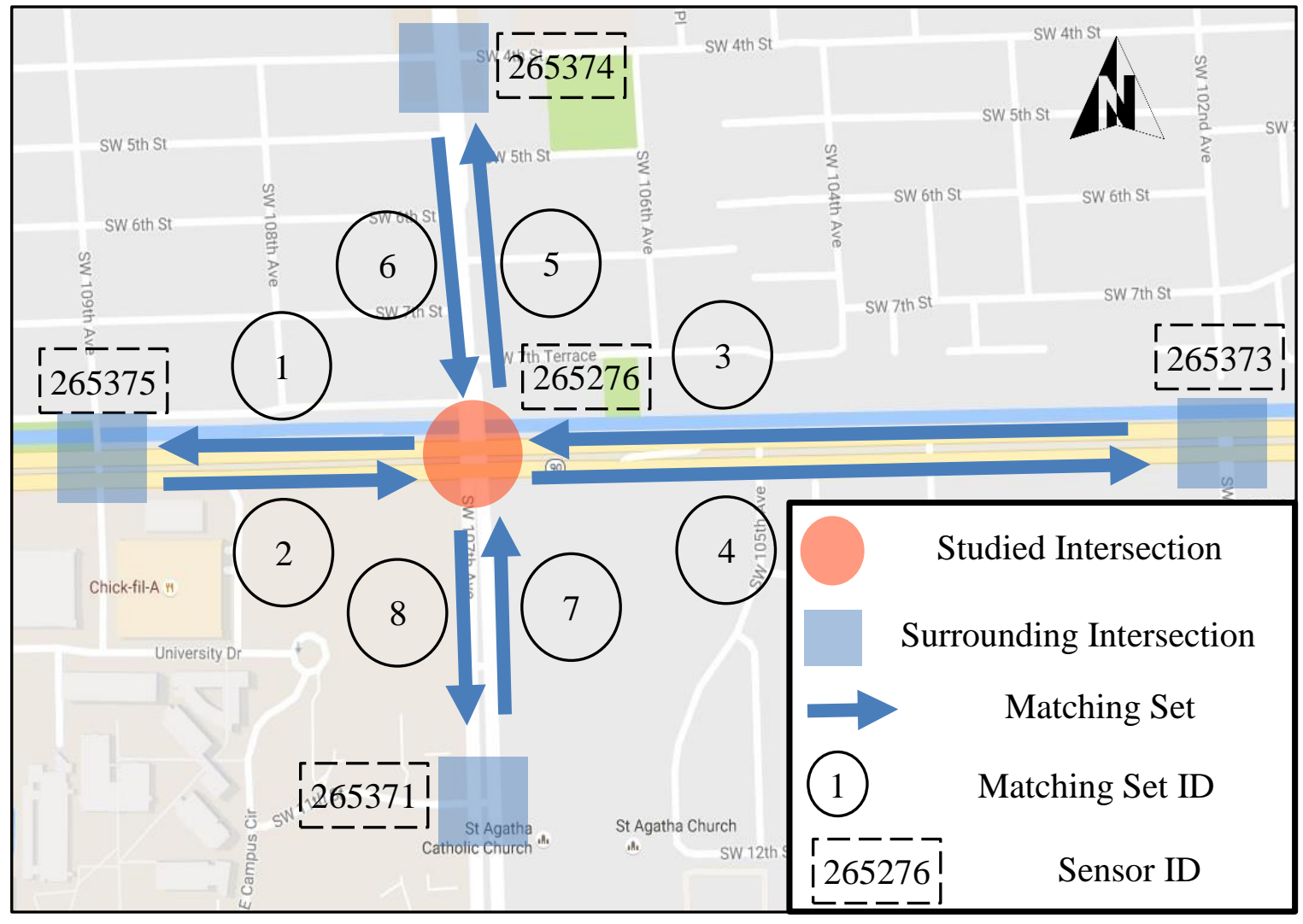

Note: Figure not drawn to scale.

Figure 3-5 Data Matching between Studied Intersection and Its Surrounding Intersections

However, it is possible that multiple intersection passages are identified from both sensors in a matching set. Using only the MAC address in the matching process will lead to a large number of mismatches. An example illustrating the correct matches and mismatches is shown in Figure 3-6. In the example, assuming there are three passages in each intersection, the three correct matches are presented as solid arrows. Figure 3-6 shows that there are six possible mismatches, as indicated by the dashed arrows, if only the MAC address is used in the matching procedure without considering the difference in the timestamps between the readings. 


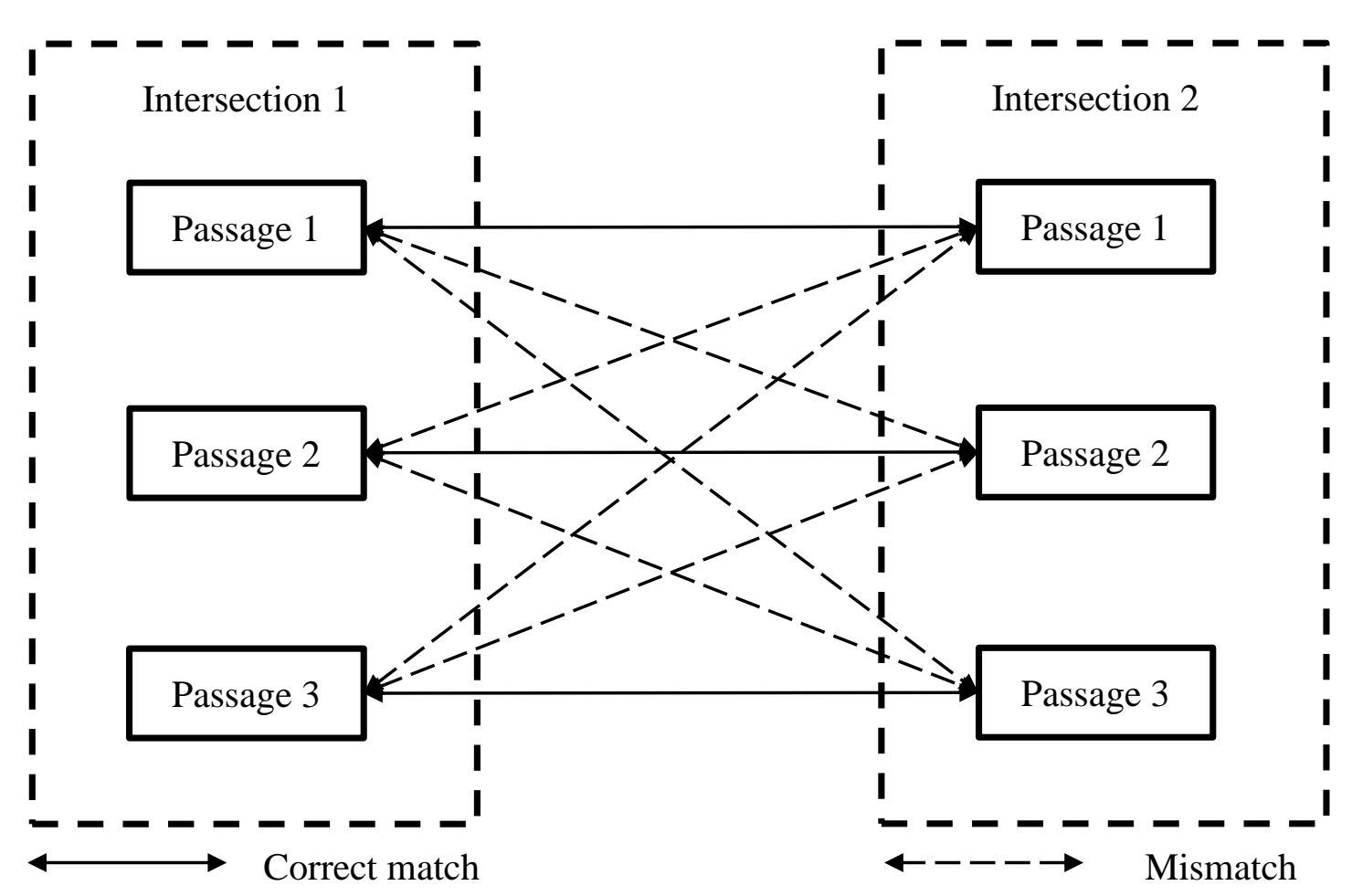

Figure 3-6 Example of Correct Matches and Mismatches for a MAC Address from Different Wi-Fi Sensors

Another example with actual data is shown in Figure 3-7 of matching between a pair of intersections, with the following information:

- the studied intersection (i.e., the intersection of Southwest 8th Street and Southwest 107th Avenue, with sensor ID 265276); and

- one of its surrounding intersections (i.e., the intersection of Southwest 8th Street and Southwest 109th Avenue, with sensor ID 265375).

A device with a MAC address shown in the figure passed the studied intersection twice on Thursday, December 3, 2015: 10:00 AM and 8:00 PM. If only using the MAC address without considering the detection time in the matching procedure, a total of four matches can be identified. However, only two matches are correct, and the other two are mismatches, due to matching the morning passage to the evening passage. Hence, the 
following is another rule that is introduced to identify the correct matches between two Wi-

Fi sensors with the same MAC address.

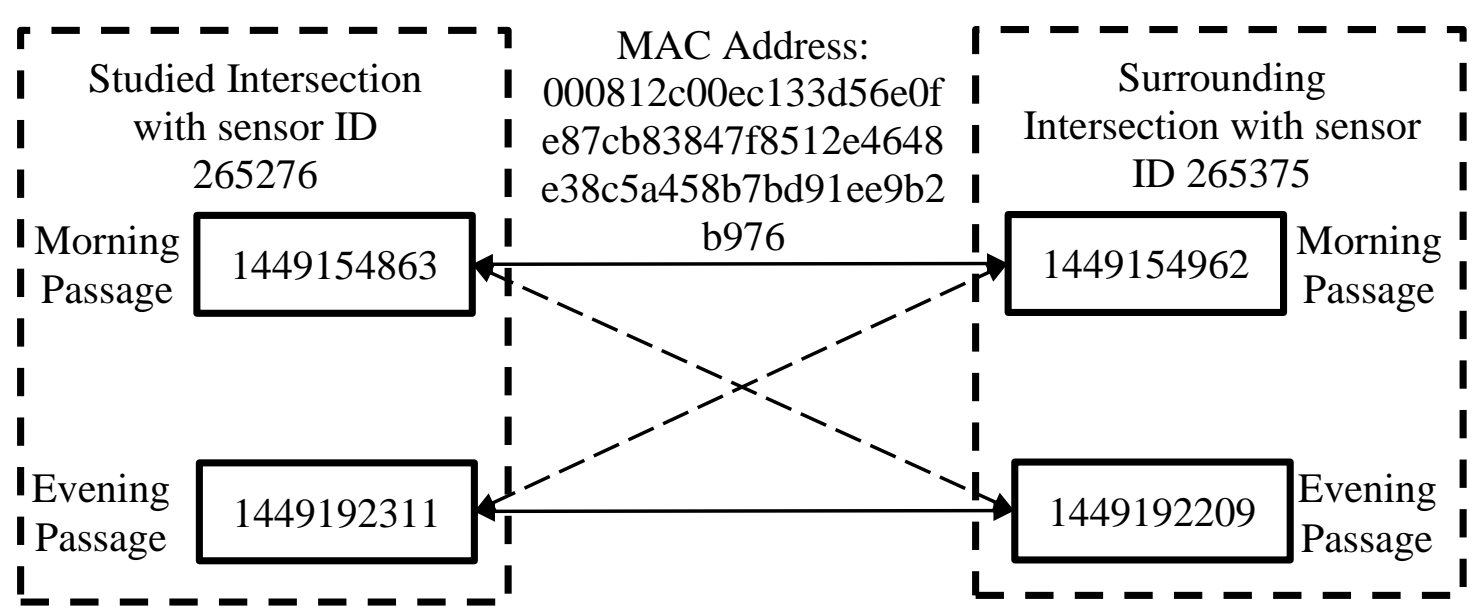

1449154863

Timestamp of the maximum

strength detection

$\longleftrightarrow$ Correct match $\longleftrightarrow----\rightarrow \quad$ Mismatch

Figure 3-7 Example of Correct Matches and Mismatches with Actual Data

Rule 2: (Identification of Trips)

Let $T_{k}$ be the $k^{\text {th }}$ trip made by a MAC address between the studied intersection $\alpha$ and a surrounding intersection $\beta . T_{k}$ is identified if:

1) The MAC address detected by the Wi-Fi sensor at $\alpha$ equals the MAC address detected by the sensor at $\beta$.

2) $\left|t_{\alpha, \max }-t_{\beta, \max }^{\prime}\right| \leq 1200$

where

$t_{\alpha, \max }=\quad$ the maximum strength detection timestamp at studied intersection $\alpha$.

$t_{\beta, \max }^{\prime}=\quad$ the corresponding maximum strength detection timestamp at a surrounding intersection $\beta$. 
The first condition in the above rule matches the MAC address from different WiFi sensors. The second condition identifies a trip during which the difference of the corresponding times (i.e., the maximum strength detection time) of the two sensors does not exceed an arbitrary threshold of 1,200 seconds (i.e., 20 minutes). The maximum strength detections (the detection when the strength of the signal first reaches the highest value) are used in the matching process since they occur when the detected devices are closest to the Wi-Fi sensors.

The purpose of adding the 20-minute time threshold to the matching algorithm is to prevent mismatches. It also avoids including extreme situations when heavy but nonrecurrent congestion occurs in the analysis period, such as incidents, signal failures, and so on. In addition, it also helps differentiate normal trips that travel through the intersection (i.e., traversals) from those that made intermediate stops for other purposes near the intersection. For example, vehicles that drove through the fast food restaurants near the intersection made intermediate stops and took a longer time to travel through the intersection. Since the threshold of 20 minutes is arbitrary and indefinite, additional filtering methods are applied to further distinguish traversals from other trips, as discussed in a later section.

As shown in Figure 3-5, the matches between pairs of intersections are directional. The direction of an identified trip from Rule 2 is introduced in the following rule.

Rule 3: (Direction of Trips)

Given a trip $T, t_{\alpha, \max } \in T$ and $t_{\beta, \max }^{\prime} \in T, T$ is traveling from the studied intersection $\alpha$ to a surrounding intersection $\beta$ if it satisfies the conditions in Rule 2 and the condition below, and vice versa. 


$$
t_{\alpha, \max }-t_{\beta, \max }^{\prime}<0
$$

The device, in tandem with a trip, always reaches one intersection first, then the other. Therefore, the UNIX timestamp of the maximum strength detection at the first reached intersection is always less than at the second reached intersection in a matching set.

As shown in Figure 3-5., all matching sets end or begin at the studied intersection of Southwest 8th Street and Southwest 107th Avenue. Therefore, previously matched trips can be associated with turning movements by matching the MAC addresses and timestamps between an approaching link and a departing link at the studied intersection. Similar to the previous matching procedure, the maximum strength detections are utilized in the turning movement matchings. The rule of turning movement identification is given as follows.

\section{Rule 4: (Identification of Turning Movements)}

A trip is associated with a turning movement $T M$ if:

1) The MAC address of a trip $T$ that ended at the studied intersection $\alpha$ equals the MAC address of a trip $T^{\prime}$ that started at the same intersection. $t_{\alpha, \max } \in$ $T$ and $t_{\alpha, \max }^{\prime} \in T^{\prime}$.

2) $t_{\alpha, \max }=t_{\alpha, \max }^{\prime}$

For example, as shown in Figure 3-5, if a MAC address of an identified trip $T$ from the matching set 2 (i.e., an approaching link) is found from an identified trip $T^{\prime}$ from the matching set 5 (i.e., a departing link), and $t_{\alpha, \max }$ at the studied intersection $\alpha$ of trip $T$ 
equals to $t_{\alpha, \max }^{\prime}$ at the studied intersection $\alpha$ of trip $T^{\prime}$, the two trips are associated with an eastbound left-turn (EBL) movement.

With the association of the matchings with intersection turning movements, it is possible to conduct analyses of the performance of various turning movements. Travel times for the trips associated with various turning movements can be calculated as the difference between the timestamps of the readings at two intersections. As discussed previously, travel times can be used to distinguish traversals from other trips. As shown in Section 3.2 , there are four stages of detection: First, Last, Maximum Strength, and Others. The first three stages of each intersection can be used in the analysis, resulting in as many as nine combinations of potential methods to match and estimate travel times between two intersections (i.e., $3^{2}=9$ ). However, the following five types of matched travel times are the most popular:

- First-to-first detection travel time

- First-to-last detection travel time

- Max-strength-to-max-strength detection travel time

- Last-to-first detection travel time

- Last-to-last detection travel time

Figure 3-8 illustrates the five types of matched travel times at various stages of detection between the upstream intersection and the studied intersection. In this dissertation, the travel time for a trip is calculated as the difference between the last detection at the upstream intersection and that of the studied intersection, or the last-to-last detection travel time. As can be seen in Figure 3-8, utilizing the last-to-last detection in the travel time calculation is the best way to eliminate the impacts from the upstream signal at the 
beginning of the trip. Vehicles should have already passed the upstream intersection when the last detections were recorded by the upstream sensor. It also ensures that the influence of the studied signal during the trip is retained, as the vehicles should have traveled through the studied intersection between the last-to-last detections. In addition, the last-to-last detection avoids the impacts from the downstream signal. Vehicles should be a certain distance away from the downstream intersection when the last detections were recorded by the sensor at the studied intersection. The calculated travel times were then used for further data filtering in order to distinguish trips made by traversing vehicles.

Therefore, the travel time of an identified trip $T$ can be calculated with the following equation.

$$
t t_{\alpha, \beta}=\left|t_{\alpha, l a s t}-t_{\beta, l a s t}\right|
$$

where

$t t_{\alpha, \beta}=\quad$ the travel time between the studied intersection $\alpha$ and a surrounding intersection $\beta$, in the unit of seconds.

$t_{\alpha, \text { last }}=\quad$ the last detection timestamp at the studied intersection $\alpha$ of an identified trip $T$.

$t_{\beta, \text { last }}=\quad$ the last detection timestamp at the surrounding intersection $\beta$ of $T$.

It is worth noting that the calculated travel time from Equation 3-1 is always positive, since the direction of an identified trip in the analysis is constrained by Rule 3 and the trip is associated with the corresponding turning movement by Rule 4 . 


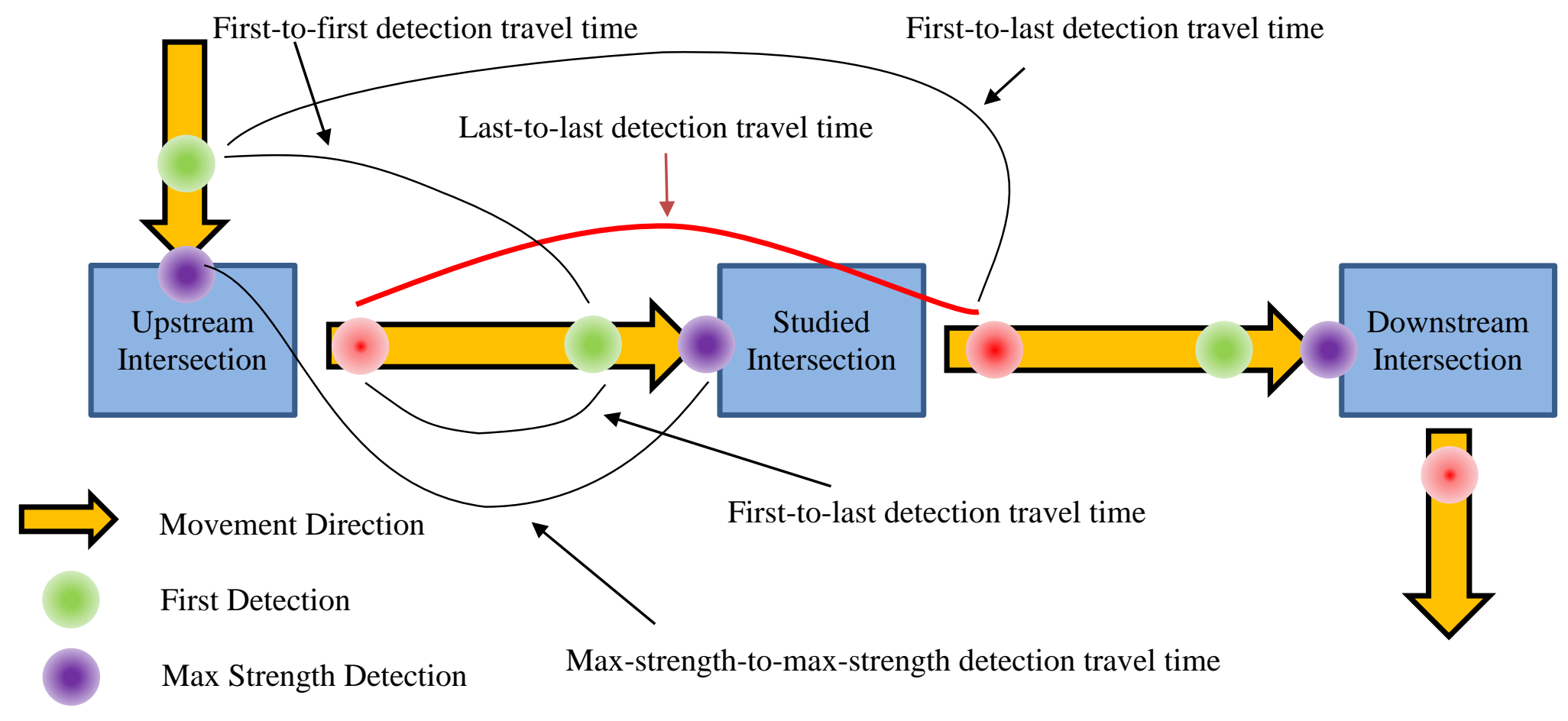

Last Detection

Figure 3-8 Illustration of Different Types of Matched Travel Times 


\subsubsection{Data Filtering}

Traversals at the studied intersection can be distinguished from other trips with the calculated last-to-last detection travel times. The travel times of the traversals associated with different turning movements should neither be too long or too short. Prolonged travel times may belong to devices with different transportation modes or those that made intermediate stops for other purposes. They can possibly also exist due to extreme nonrecurrent congested conditions, which are not the targets of the method developed in this dissertation. On the other hand, extremely short travel times may belong to errors associated with Wi-Fi sensor technologies, or associated with their inability to identify the exact locations of the readings. Extremely short travel times may lead to inaccurate diagnosis results on the traffic signal operation.

To prevent the negative impacts of extremely short travel times on distinguishing traversals, trips with travel times shorter than the presumed shortest travel time at each movement are filtered out. The presumed shortest travel time $t t_{\alpha, \beta, \min }$ in seconds is calculated in the equation below.

$$
t t_{\alpha, \beta, \min }=\frac{L_{\alpha, \beta}}{S L+5} \times 3600
$$

where

$t t_{\alpha, \beta, \min }=\quad$ shortest travel time between the studied intersection $\alpha$ and a surrounding intersection $\beta$, in the unit of seconds.

$L_{\alpha, \beta}=\quad$ the distance between the two intersections, in the unit of miles.

$S L=\quad$ the posted speed limit of the roadway segment between the two intersections, in the unit of miles per hour. 

limit, in the unit of miles per hour.

Equation 3-2 defines the shortest travel time between the two intersections as the travel time spent by a vehicle that is running five miles per hour above the posted speed limit. All identified trips with the travel time less than the presumed shortest travel time are treated as outliers and filtered out from the dataset. A rule of that filtering procedure is given below.

Rule 5: (Identification of Traversal Candidates)

A trip $T$ is a traversal candidate if:

$$
t t_{\alpha, \beta} \geq t t_{\alpha, \beta, \min }
$$

where

$t t_{\alpha, \beta}=\quad$ the travel time between the studied intersection $\alpha$ and a surrounding intersection $\beta$, calculated from Equation 3-1.

$t t_{\alpha, \beta, \min }=\quad$ the presumed shortest travel time between the studied intersection $\alpha$ and a surrounding intersection $\beta$, calculated from Equation 3-2.

Figure 3-9 shows an example of all traversal candidates with the application of Rule 1 to Rule 5. In this example, one month's Wi-Fi readings are analyzed, and travel times in different time periods of the day are plotted in the figure. A day is evenly divided into 48 time intervals, with each interval being exactly 30 minutes long. The distance between the upstream intersection and the studied intersection for the northbound thru (NBT) movement is 1,000 feet, and the posted speed limit is 40 miles per hour. Thus, the presumed shortest travel time between the two intersections is around 15 seconds, according to Equation 3-2. 


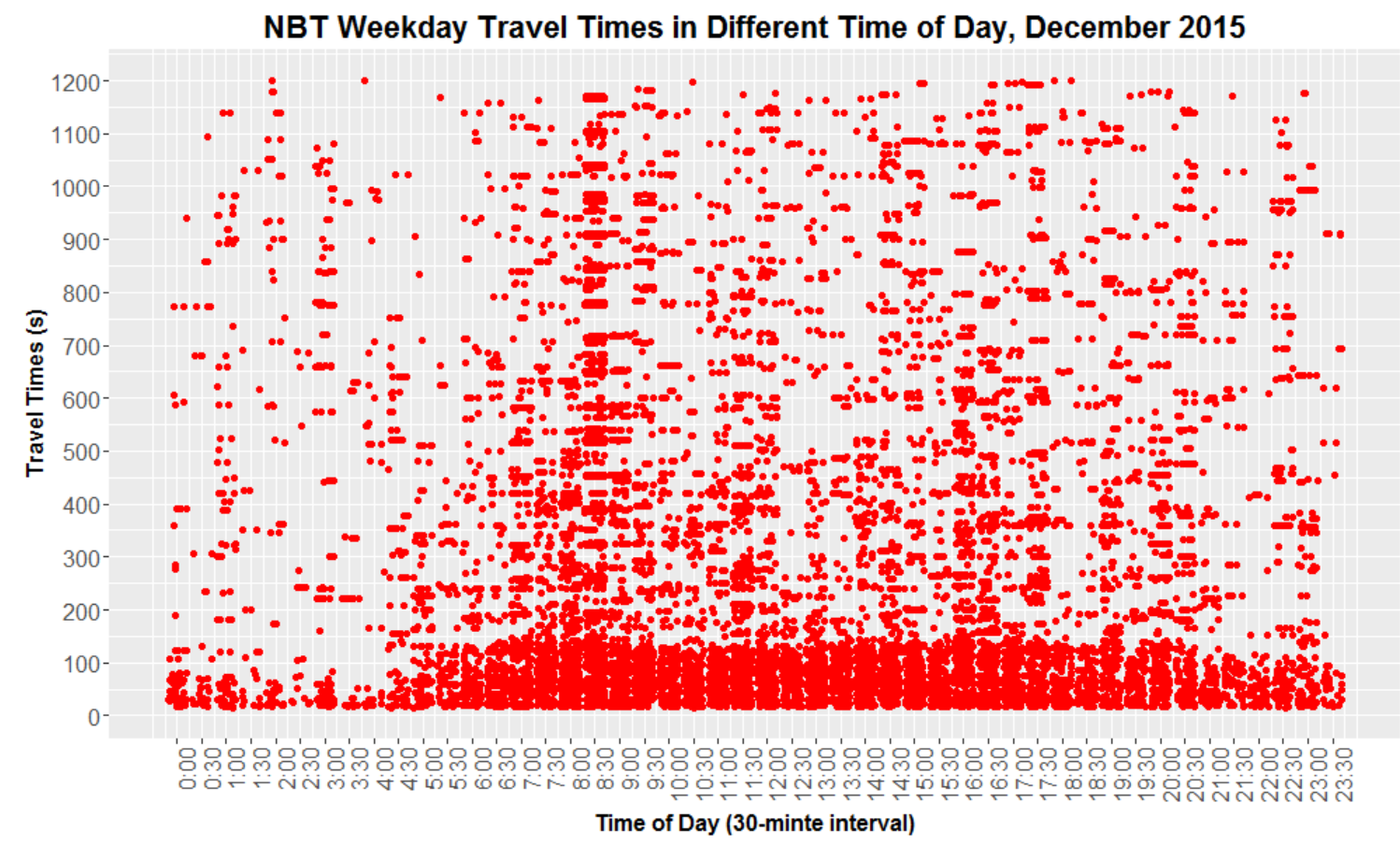

Figure 3-9 Example Travel Times with Application of Rule 1 to Rule 5

The travel time points in Figure 3-9 seem to be dispersed and cover a wide range from the presumed shortest travel time (i.e., 15 seconds) to the upper boundary (i.e., 1,200 seconds). However, it can be seen that the majority of the data points are concentrated together in the lower part of the plot, approximately below 200 seconds. An artificial line is added to the travel time plot and presented in Figure 3-10. Densities appear to be different on the two sides of the added blue dashed line, and points seem to be denser below the line. In this context, a cluster analysis is reasonably applied to the data.

A cluster analysis or clustering can group objects into different clusters so that the objects in the same cluster are similar to each other yet different from the objects in other clusters (Tan et al. 2006). Based on the example above, the points in a cluster should have similar distance between each other if the cluster analysis is applied. In other words, the densities in various clusters are expected to be different. Therefore, a clustering algorithm 
that focuses on density similarity, or DBSCAN (Density-Based Spatial Clustering of Applications with Noise), is applied in an attempt to identify traversals from other trips.

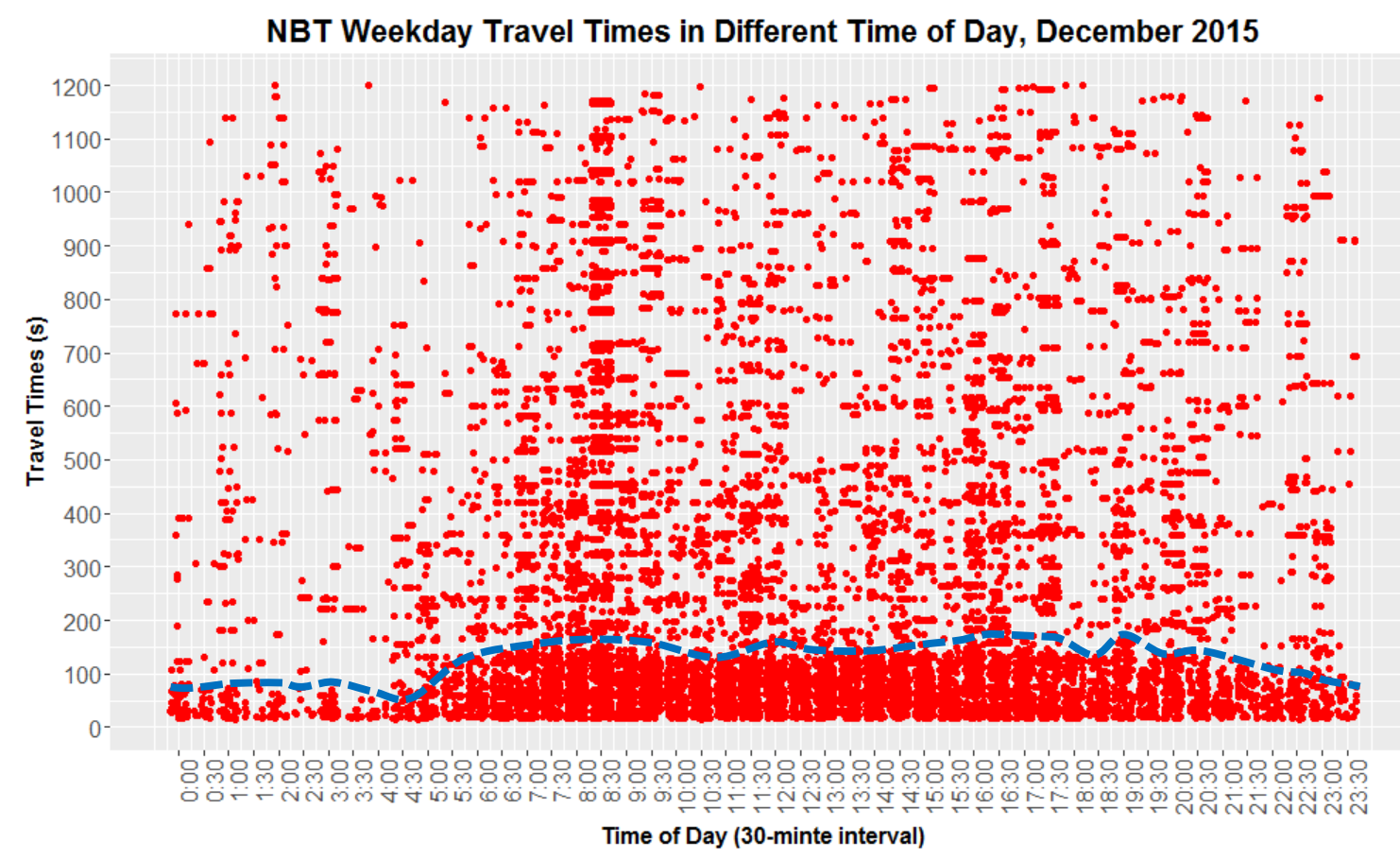

Figure 3-10 Example Travel Times with Artificial Dividing Line

In order to identify different dense regions, DBSCAN requires two parameters:

1. epsilon, $\varepsilon($ eps $)$

2. minimum points (MinPts)

$\varepsilon$ defines the radius of a point's neighborhood and MinPts finds the minimum density of the region within the radius of the point $(\varepsilon)$. To be defined as a cluster, a group of points must satisfy two properties:

1. All points must be within a region (circle), of which the radius equals to $\varepsilon$.

2. The number of points in the region must exceed the MinPts. 
Essentially, DBSCAN finds the groups of points or clusters, which are denser than the preferred minimum density. With the appropriate parameters, clusters can be identified, as well as the outliers that are not in any of the clusters.

Figure 3-11 shows an example of clusters identified by the DBSCAN algorithm, with the noises (outliers) presented using black solid dots. A large number of clusters are identified with similar densities. However, not all of them belong to traversals. Different clusters represent a group of detected devices that have similar travel time characteristics. There are numerous possibilities, such as pedestrians, bicycles, vehicles stopped at fast food restaurants, vehicles stopped at an ATM, etc. In order to identify traversals only, the following assumptions are made.

Assumption 1: (The Fastest Transportation Mode)

With the purpose of traversing the intersection, traveling in a vehicle is the fastest transportation mode between a surrounding intersection and the studied intersection.

Assumption 2: (Identifying Clusters of Traversals)

The cluster that has the shortest travel time is the cluster of traversals.

Figure 3-12 presents the identified traversals from other trips based on the above assumptions. Only the cluster that has the shortest travel time is identified as the group of traversals. Almost all identified traversals have lower travel times compared to other trips, except a few between 2:30 AM and 3:30 AM. Moreover, the results meet the expectation that the points below the dividing line in Figure 3-10 are distinguished as traversals from other trips.

Nevertheless, it is possible that multiple clusters should be selected in some situations so that all valid traversals will be counted, especially when cycle failures are 
observed in the field. In cases where the cycle failure occurs, there will be significant gaps between travel times among different groups of vehicles, according to their arrival types. For example, vehicles arriving at the studied intersection during the green time and passing through the intersection in the same cycle will have significantly shorter travel times than those arriving on red and still experiencing cycle failures. As a result, traversal vehicles will be grouped in two different clusters by the algorithm. This points to the need for the identification of multiple clusters in the DBSCAN algorithm to identify all valid traversals.

Only one cluster from the DBSCAN filtering algorithm is identified in this dissertation since there is no proof from the data, implying that multiple clusters should be included. However, future efforts are needed to investigate this issue.

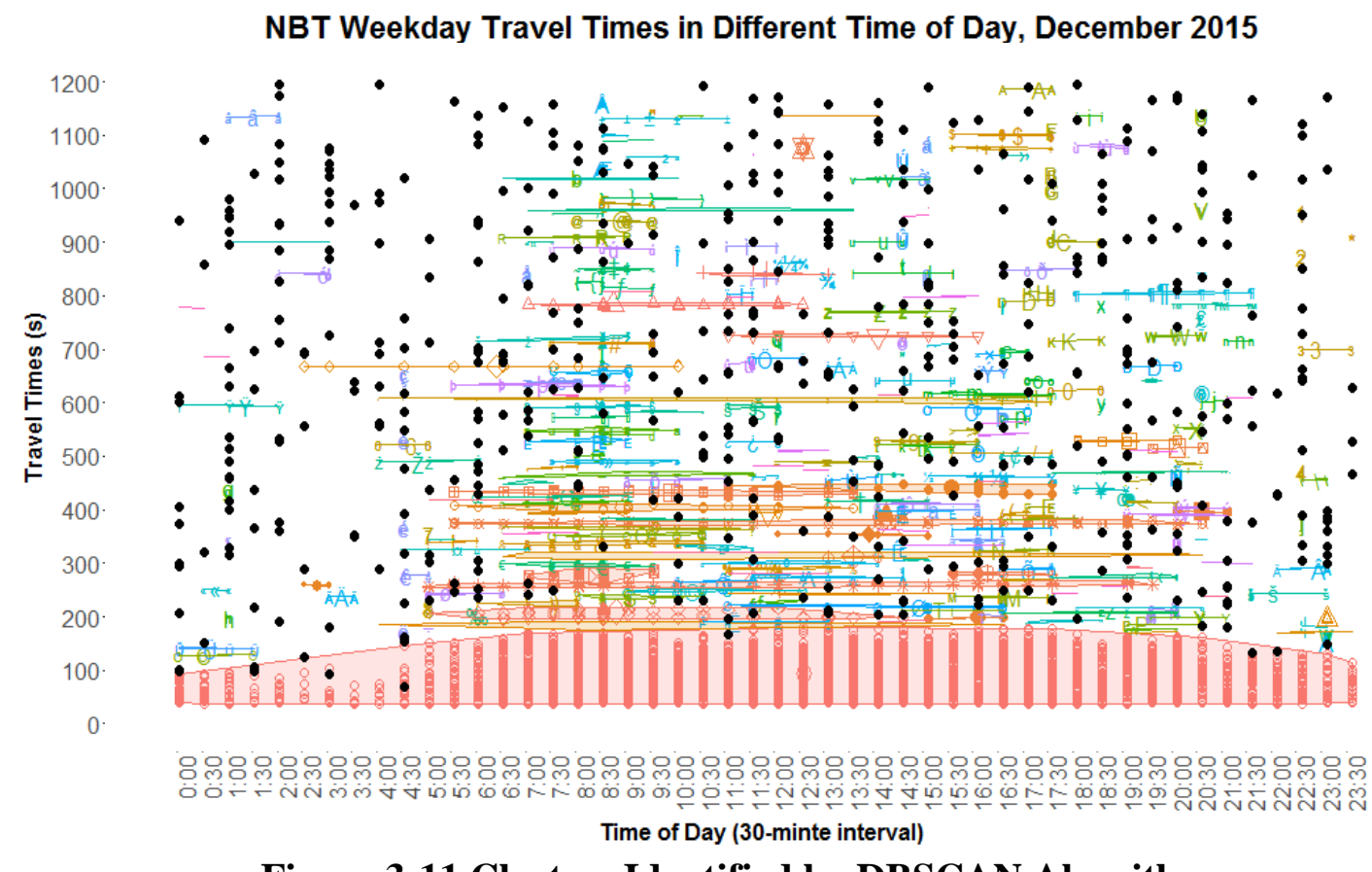

Figure 3-11 Clusters Identified by DBSCAN Algorithm

In summary, in order to identify traversals from other trips from the original readings of the Wi-Fi sensors, the following data matching and filtering procedures are applied in this dissertation: 
- A passage is identified if the difference between two consecutive timestamps in a Wi-Fi sensor for one MAC address is not more than 60 seconds.

- A trip is identified if the difference between the following timestamps is not more than 1,200 seconds for a MAC address:

o the maximum strength detection timestamp of the corresponding passage at the studied intersection

$\circ$ the maximum strength detection timestamp of the corresponding passage at a surrounding intersection

- A trip is associated with a turning movement if the MAC address and the corresponding timestamp starting/ending at the studied intersection are exactly the same as those of a trip ending/starting at the studied intersection.

- A traversal potential is identified if the difference between the following timestamps is not smaller than the presumed shortest travel time, at each movement:

○ the last detection timestamp of a trip at the studied intersection

o the last detection timestamp of the trip at a surrounding intersection

- A traversal is identified if it belongs to the cluster that has the shortest travel time with the application of the DBSCAN algorithm.

The traversals identified by the DBSCAN algorithm for each turning movement associated with a signal phase are presented from Figure 3-12 to Figure 3-19. 
NBT Weekday Traversals vs. Other trips, December 2015

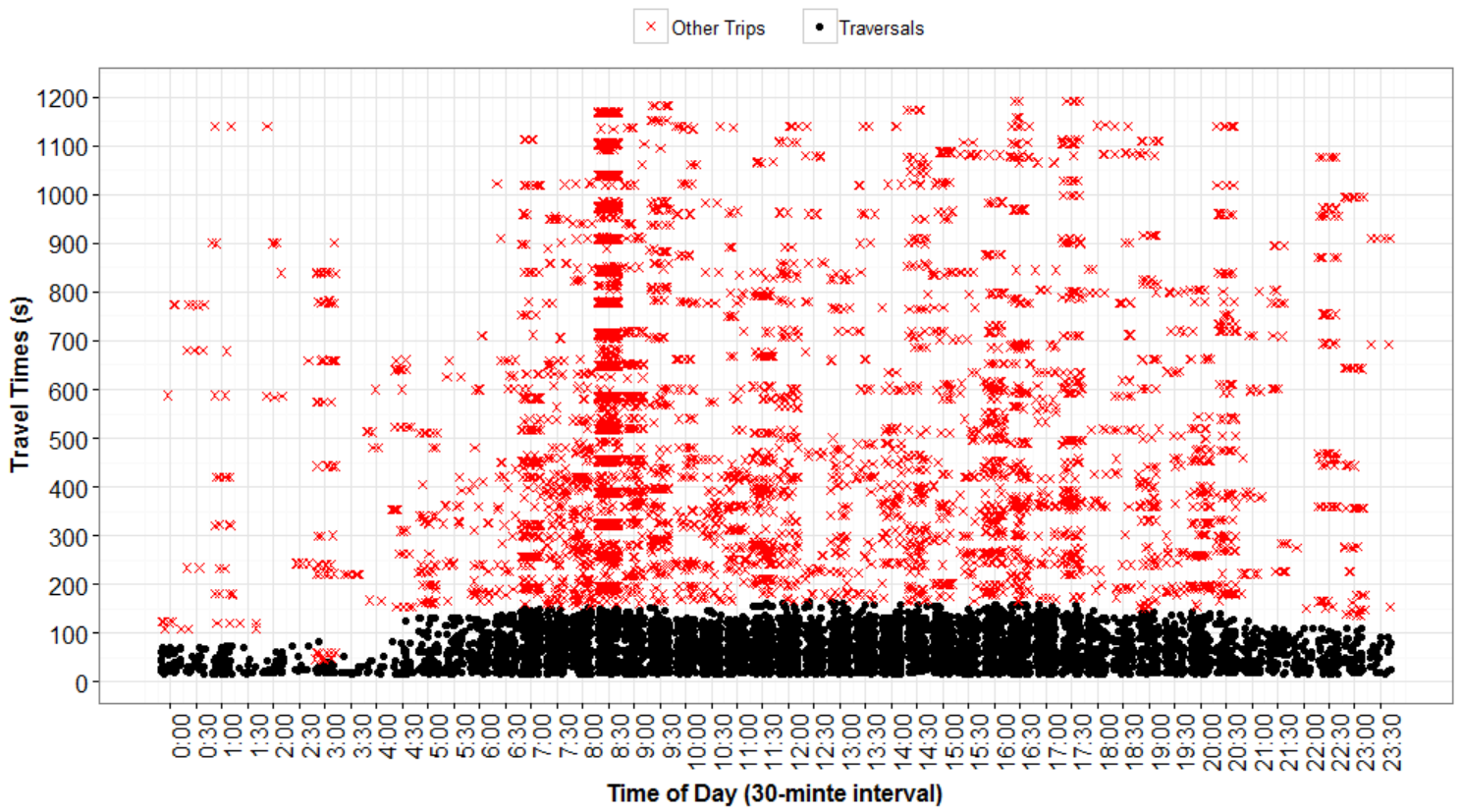

Figure 3-12 Identified Traversals and Other trips on the NBT Movement

EBL Weekday Traversals vs. Other trips, December 2015

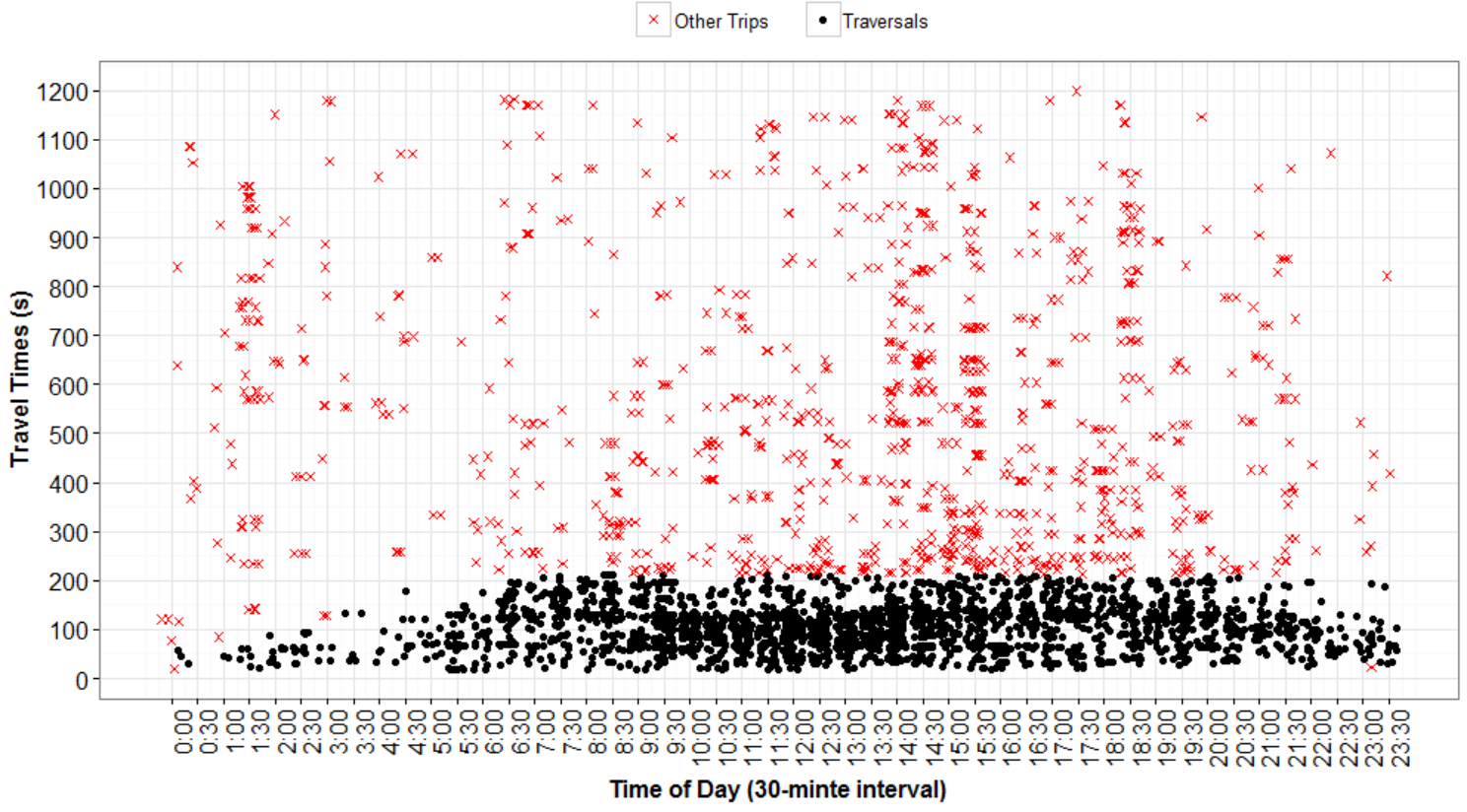

Figure 3-13 Identified Traversals and Other trips on the EBL Movement 
EBT Weekday Traversals vs. Other trips, December 2015

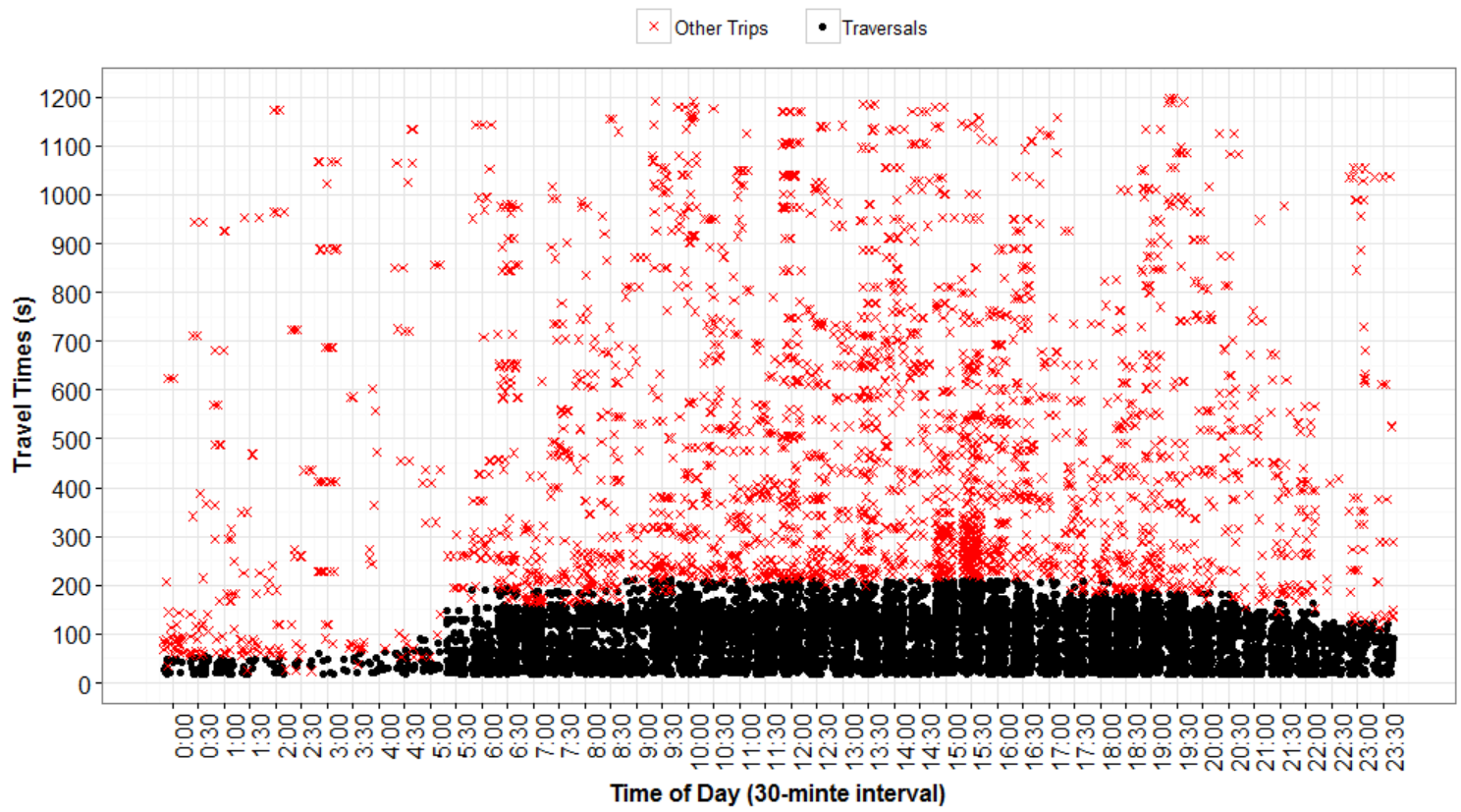

Figure 3-14 Identified Traversals and Other trips on the EBT Movement

NBL Weekday Traversals vs. Other trips, December 2015

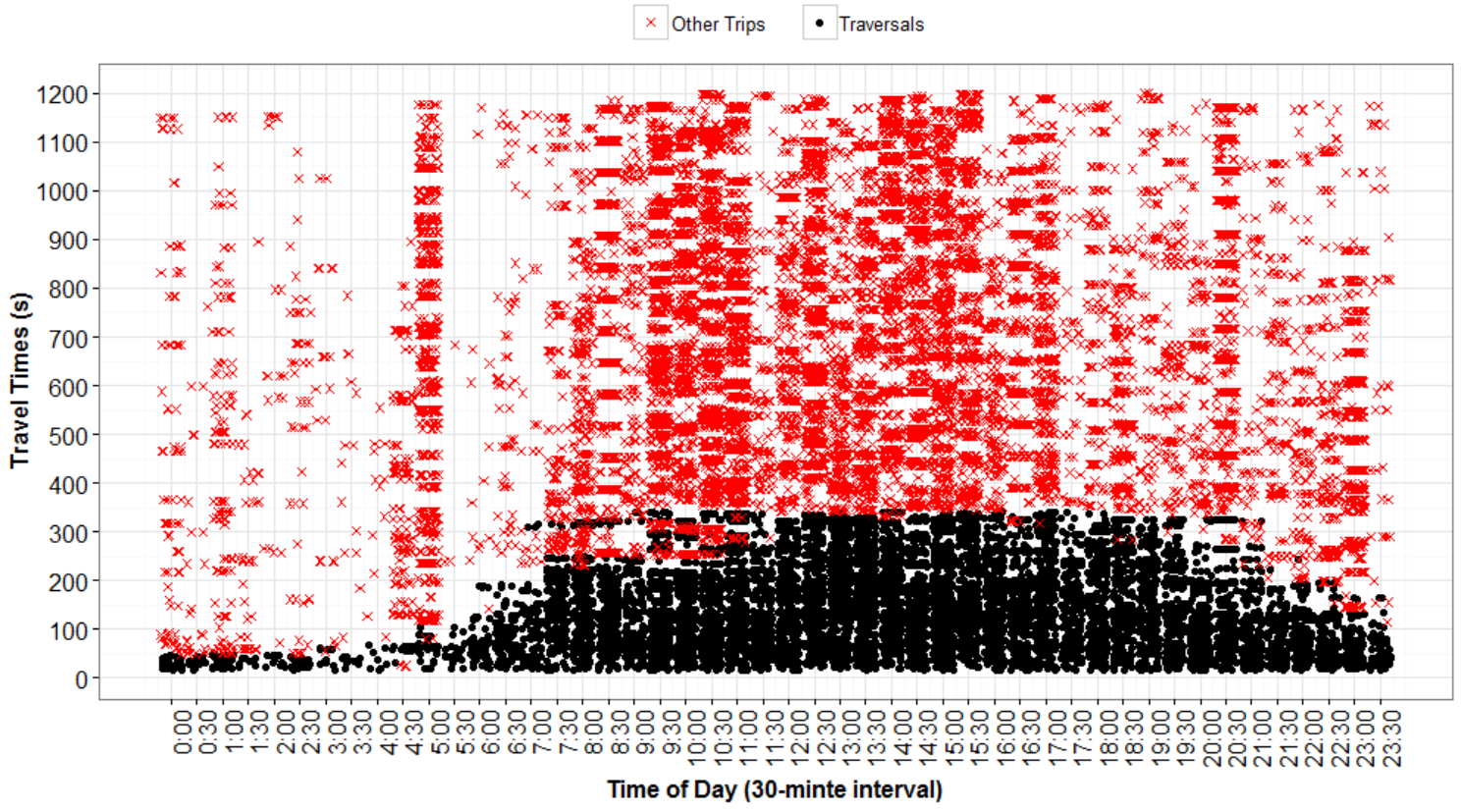

Figure 3-15 Identified Traversals and Other trips on the NBL Movement 
WBL Weekday Traversals vs. Other trips, December 2015

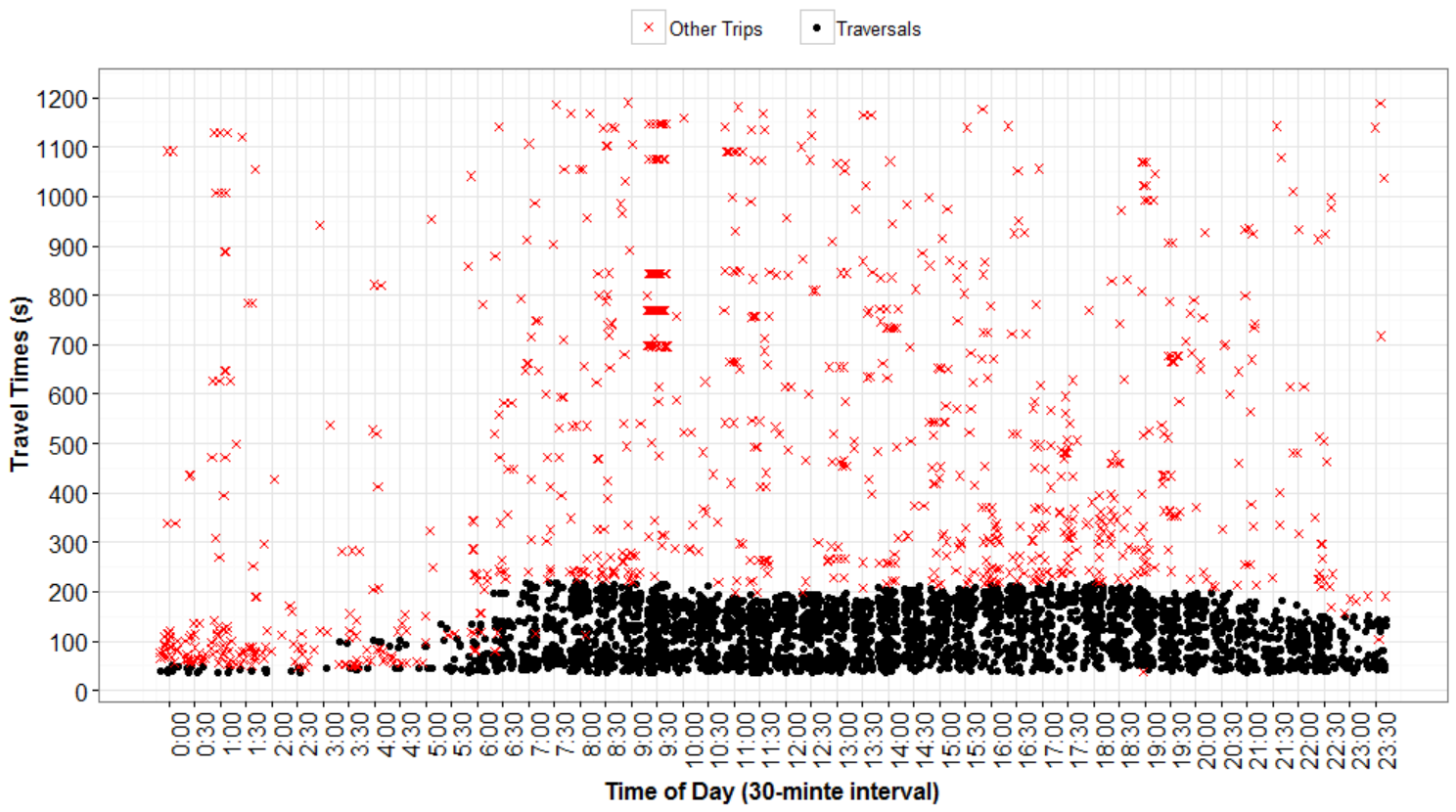

Figure 3-16 Identified Traversals and Other trips on the WBL Movement

WBT Weekday Traversals vs. Other trips, December 2015

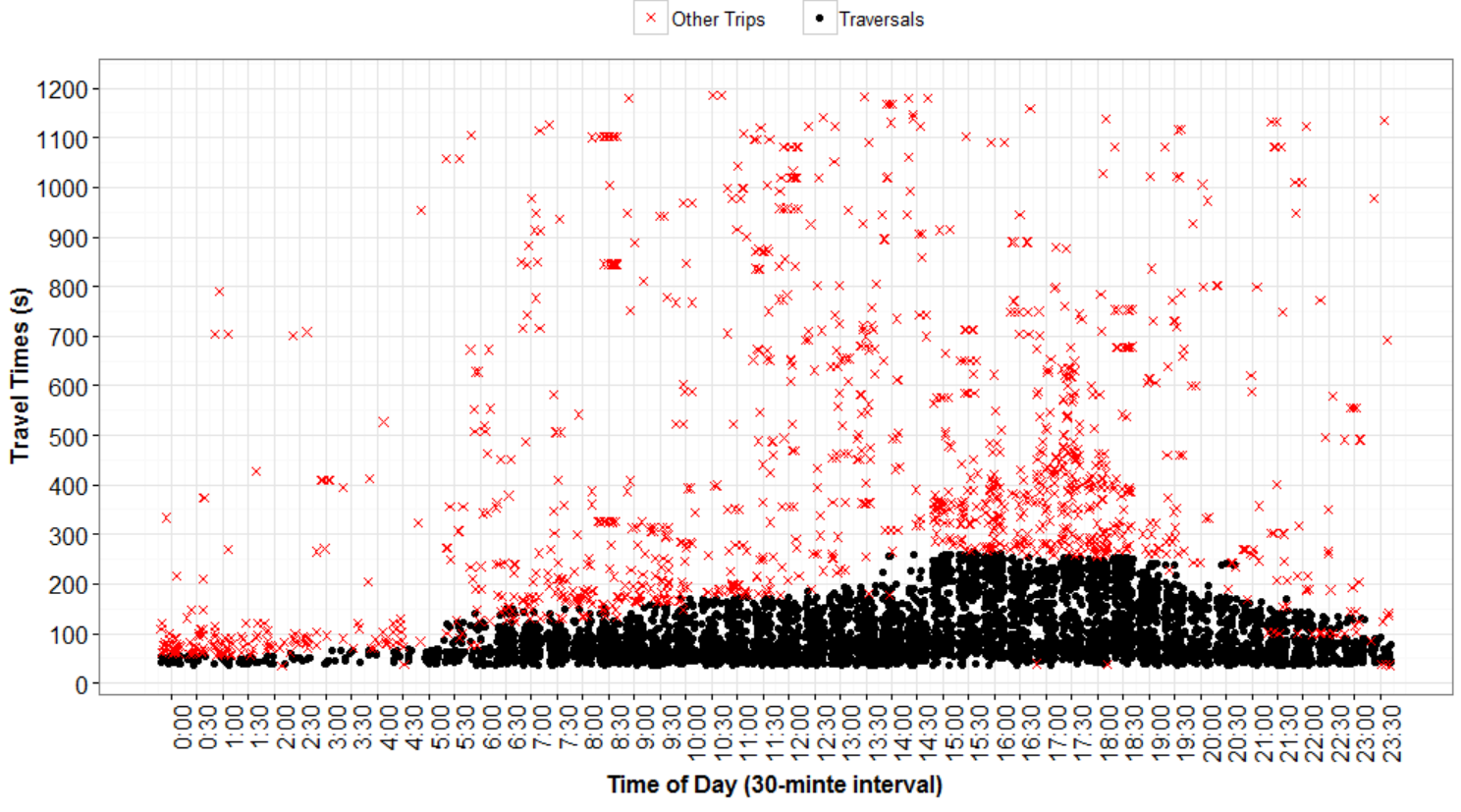

Figure 3-17 Identified Traversals and Other trips on the WBT Movement 
SBL Weekday Traversals vs. Other trips, December 2015

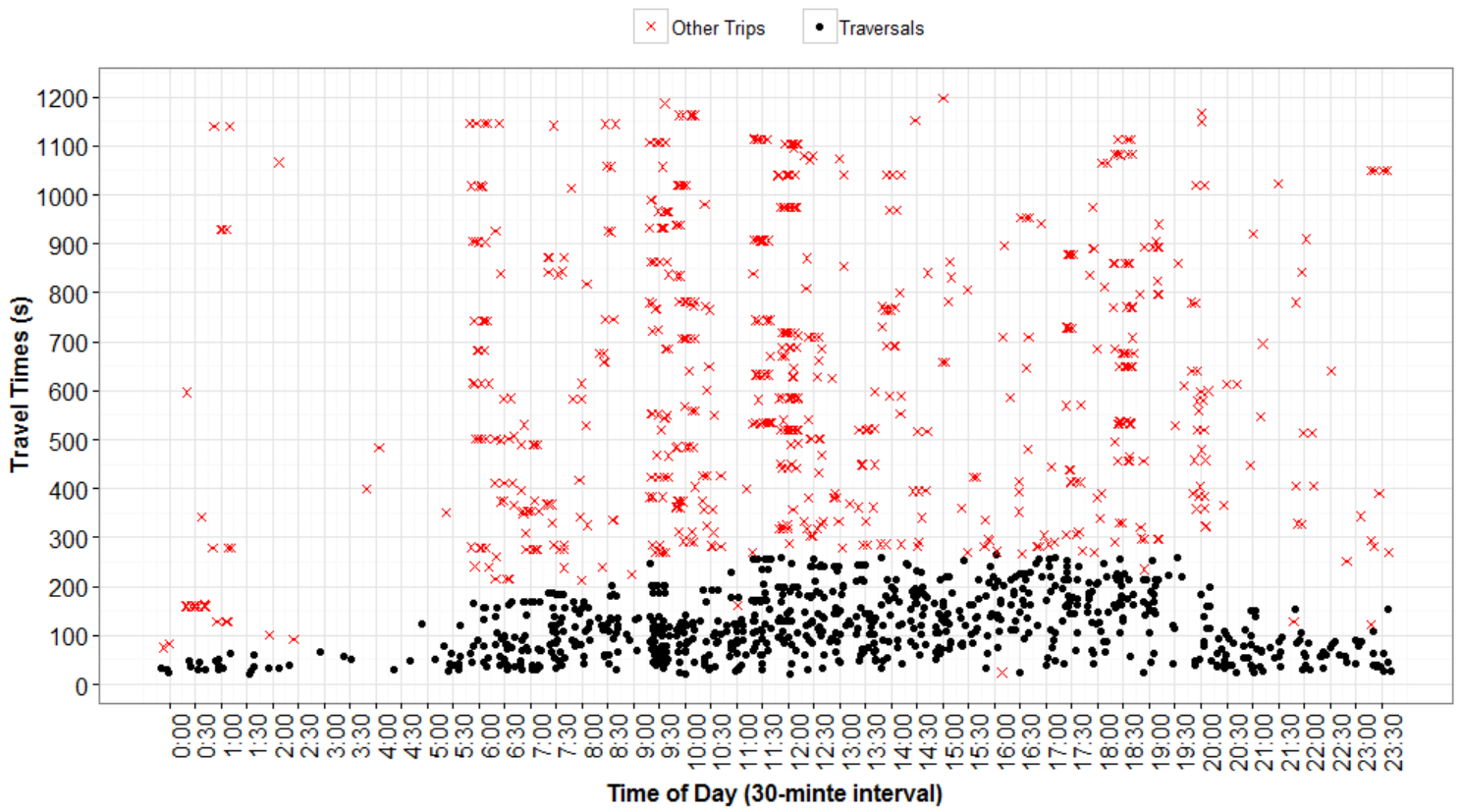

Figure 3-18 Identified Traversals and Other trips on the SBL Movement SBT Weekday Traversals vs. Other trips, December 2015

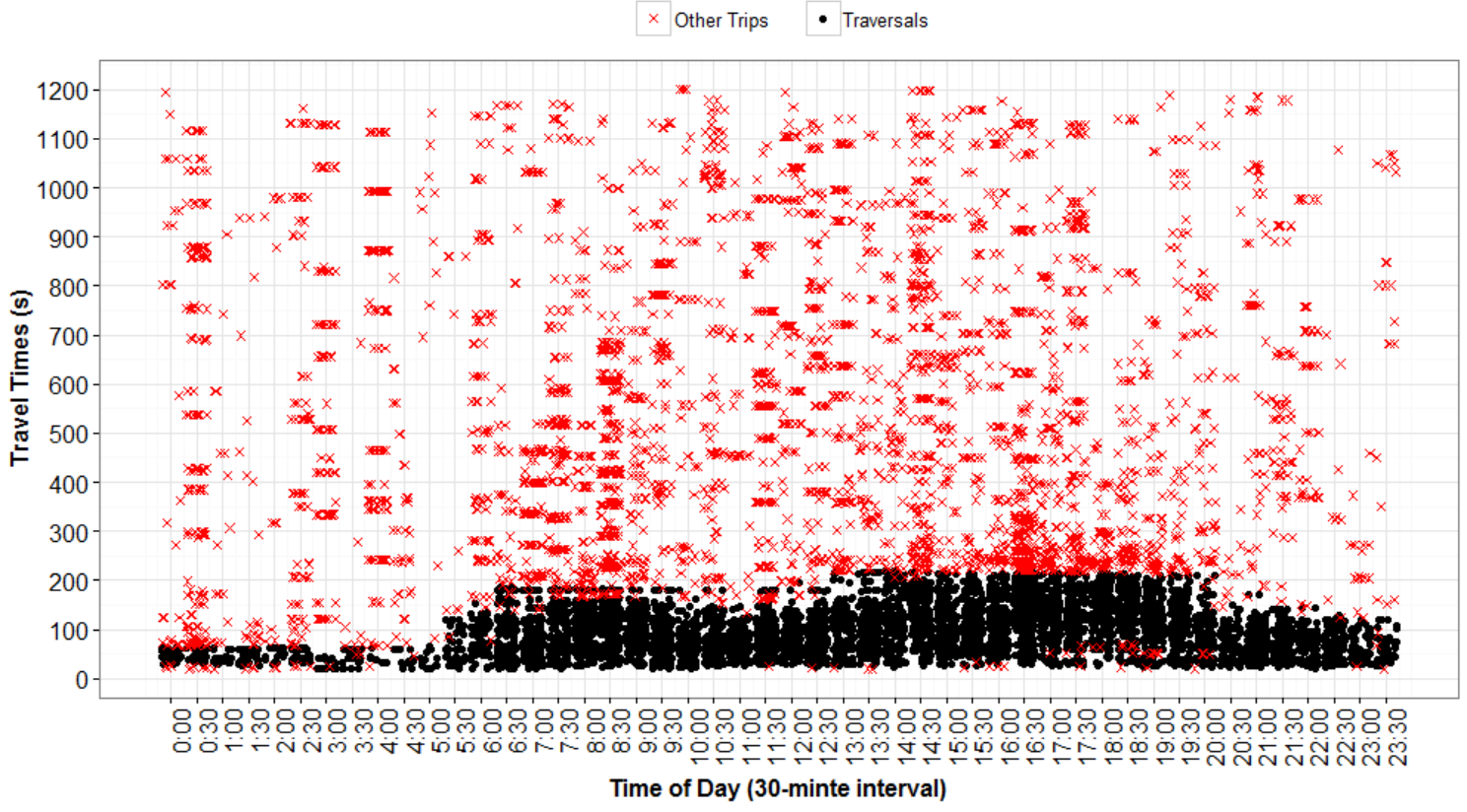

Figure 3-19 Identified Traversals and Other trips on the SBT Movement 


\subsubsection{Data Aggregation (Travel Time Representation)}

The calculated travel times for traversals at each turning movement can be represented in different ways for further utilization in the analysis. For example, it can be represented by the average travel times, percentile travel times, delays, travel time indices, and so on. Due to the link length differences and geometry diversities among various movements, the travel times may not reflect the traffic conditions correctly. A better measure is the use of delay, which is the extra time spent on travel compared to free-flow travel conditions. This measure considers the heterogeneity of the geometry attributes, since it uses the free-flow travel time as the basis, which is movement-specific. In this context, delay equals the difference between the actual travel times and free-flow travel time of a movement. As an alternative, the travel time index $(T T I)$, which is the ratio of the actual travel time to the free-flow travel time as represented in Equation 3-3, also considers the dissimilarity of the geometry conditions. TTI is a prevailing measure of effectiveness (MOE) that is used in travel time reliability studies. This measure has the added advantage in that different percentile travel times can be considered in the analysis.

$$
T T I=\frac{\text { Actual Travel Time }}{\text { Free-Flow Travel Time }}
$$

$T T I$, as is the case with delays, reflects the extra time spent on travel compared to the time spent in free-flow conditions. TTI is used in this dissertation as a main MOE for signal operation diagnoses. Instead of using an absolute value, such as delay, a ratio will give an intuitive impression of the traffic conditions.

In order to aggregate the $\mathrm{Wi}-\mathrm{Fi}$ data to reflect the traffic conditions, TTI is used to produce the Cumulative Distribution Function (CDF) plots, which are a visualization and analysis method extensively applied in the Transportation Research Board's second 
Strategic Highway Research Program (SHRP 2) L02 project for travel time reliability analysis (List et al. 2014). The CDF is a function in which the dependent variable is the probability that a variable is less than or equal to the independent variable $\mathrm{x}$. The CDF of a random variable $\mathrm{X}$ is defined in Equation 3-4 below (Cumulative Distribution Function 2016):

$$
F_{X}(x)=P(X \leq x)
$$

In this dissertation, a set of CDF plots of TTI for each movement at the studied intersection are produced based on December weekday data covering the investigated period from 5:00 AM to 8:00 PM. The TTI for each movement is calculated from the WiFi data as the ratio of the actual travel time to the estimated free-flow travel time for that movement. The utilized estimation of free-flow travel time for each movement is selected as the $15^{\text {th }}$ percentile of all travel times during the day, which are calculated based on the measurements of valid traversals of that movement. The $15^{\text {th }}$ percentile travel time indicates the travel time that is faster than the travel times of 85 percent of traffic. This is a commonly used method of selecting representative free-flow speed/travel time of traffic. Different estimation of free-flow travel times in the proposed framework of traffic signal operation diagnosis can be selected by decision-makers based on their needs and experience.

An example of the CDF plots is shown in Figure 3-20. The TTI values of the eastbound left-turn (EBL) movement of the studied intersection are summarized by hours and presented in three different graphs based on the time of day: morning (from 5:00 AM 
to 9:59 AM), midday (from 10:00 AM to 2:59 PM), and afternoon (from 3:00 PM to 7:59 $\mathrm{PM})$.

In terms of a $\mathrm{CDF}$, the higher the cumulative probability at lower TTI values, the better the TTI performance. These lower values indicate that the majority of vehicles spend shorter times to traverse the intersection. In the examples shown in Figure 3-20 for the EBL movement, the best travel time performance is between 5:00 AM and 5:59 AM. All percentiles of the TTI are below 2.5 for this time period due to the low traffic volume. The worst performance is between 8:00 $\mathrm{AM}$ and 8:59 $\mathrm{AM}$, with an $80^{\text {th }}$ percentile TTI around 3. This may due to an observed queue spillback from a downstream intersection (i.e., the intersection of Southwest 4th Street and Southwest 107th Avenue, as shown in Figure 3-3). During that hour, the City of Sweetwater police officers take control of the downstream intersection next to the Sweetwater Elementary School and give higher priority to pedestrians. Other than the time between 8:00 AM and 8:59 AM, the TTI of the EBL movement performs well, and the quality of the performance seems consistent.

Figure 3-21 through Figure 3-24 show the CDF plots of all turning movements. The hours marked with the black dotted CDF curves in the abovementioned figures indicate the time periods that have relatively better TTI performance, as the majority of cumulative probabilities stay at the lower TTI. The hours when the TTI performances are significantly worse than the rest of the day are highlighted in different colors and dotted shapes.

The following TTI performance can be interpreted for the east-west direction, as shown in Figure 3-21 and Figure 3-22:

- The TTI performance of the EBL movement is the worst between 8:00 AM and 8:59 AM. 
- The worst TTI performance of the eastbound thru (EBT) movement, as indicated by Figure 3-21, is between 3:00 PM and 3:59 PM, which is highlighted in red.

- The westbound left-turn (WBL) movement has worse TTI than the EBL movement, with particularly high TTI in the afternoon hours; for example, between 3:00 PM and 3:59 PM, 4:00 PM and 4:59 PM, and 6:00 PM and 6:59 PM.

- High TTI of the westbound thru (WBT) movement occurs between 3:00 PM and 6:59 PM.

- The TTI performance of the WBT movement from 7:00 PM and 7:59 PM improves slightly but is still worse than the hours before 3:00 PM.

It is interesting to note that the TTI of the WBT movement is the worst between 3:00 PM and 3:59 PM. The EBT also has the worst TTI performance during this period. This indicates that the pre-PM peak plan between 3:00 PM and 3:59 PM is not suitable for the main street traffic, and the PM plan needs to start earlier.

The interpretation for the north-south direction in Figure 3-23 and Figure 3-24 can be summarized as follows:

- The TTI performance of the northbound left-turn (NBL) movement, as shown in Figure 3-23, does not perform well after 11:00 AM. The CDF plots of NBL between 11:00 AM and 7:59 PM indicates a high percentage of vehicles taking a longer time to traverse the intersection.

- With regards to the NBT movement, the movement performs slightly worse between 8:00 AM and 8:59 AM, compared to the rest of the studied time periods. This can be due to the backup from the intersection of Southwest 4th Street and Southwest 107th Avenue, as mentioned earlier. 
- As shown in Figure 3-24, the southbound left-turn (SBL) movement experiences high travel times past 12:00 PM, particularly during the PM peak period between 4:00 PM and 7:59 PM.

- The southbound thru (SBT) movement has the worst performance between 1:00 PM and 7:59 PM, and between 8:00 AM and 8:59 AM. The latter may be due to police operations at the upstream signal in the school area.
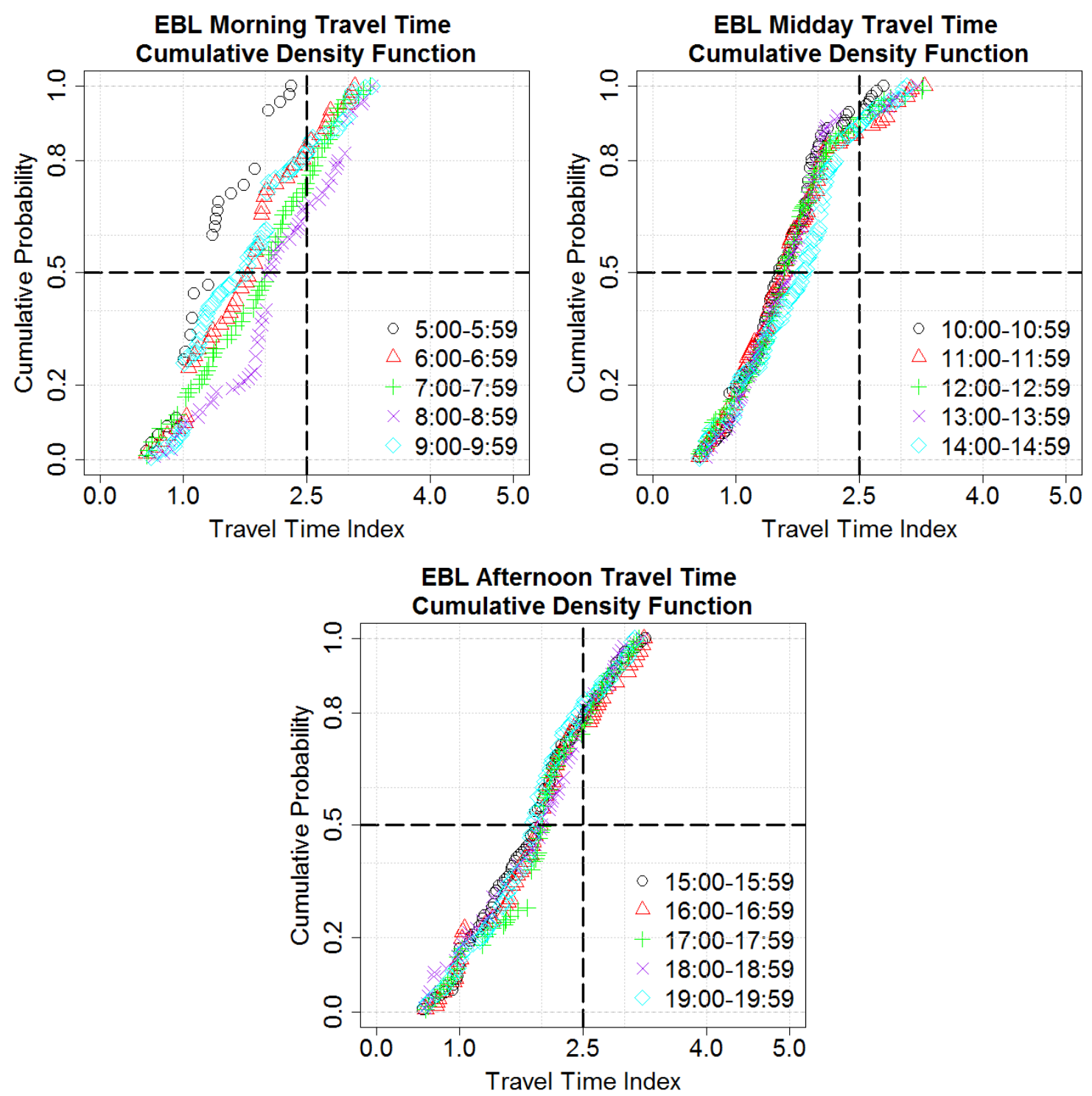

Figure 3-20 Cumulative Density Functions by Hours for Eastbound Left-turn Movement 

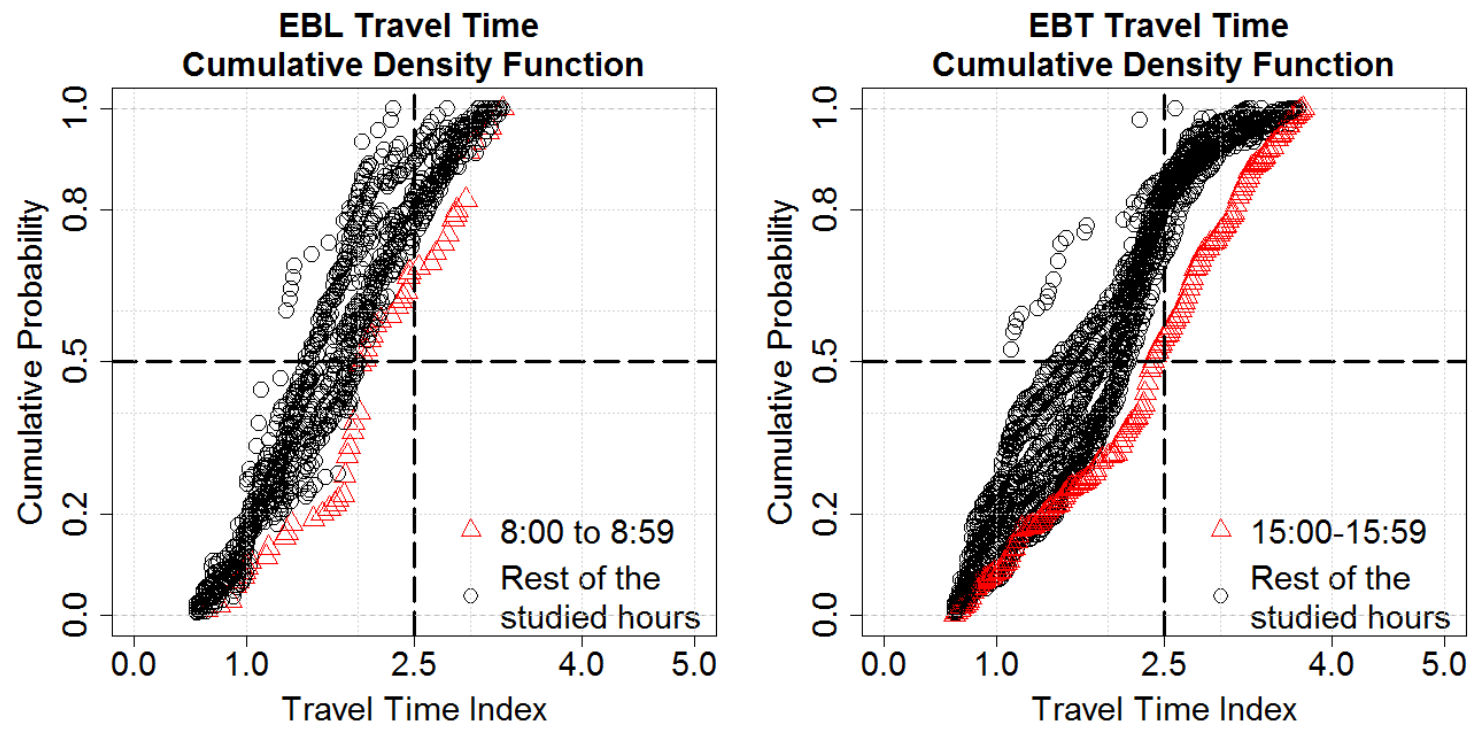

Figure 3-21 Cumulative Density Functions by Hours for Eastbound Movements.
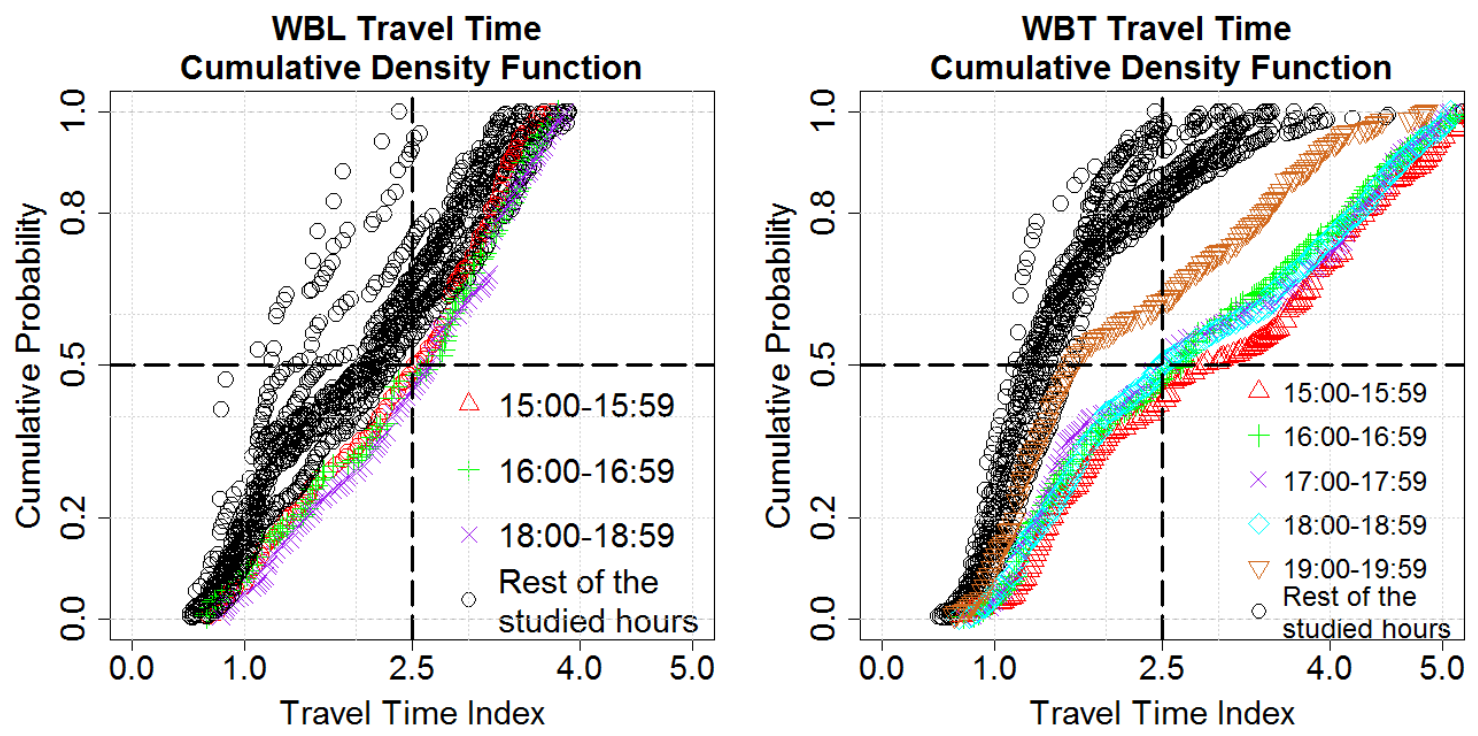

Figure 3-22 Cumulative Density Functions by Hours for Westbound Movements.

In summary, the following can be concluded based on the CDF plot interpretations:

- The WBT performance worsens in the PM peak period, starting at 3:00 PM.

- The SBL and SBT movements experience high travel times, starting at midday.

- The NBL performance is inferior most of the day, particularly starting around 11:00 AM. 
- In the PM peak period after 4:00 PM, there should be an opportunity to take some green time from the EBL and EBT and add it to the WBT, SBT, SBL, and possibly the NBL.

- At midday before 3:00 PM, the SBL and NBL movement should receive more green time, which can potentially be taken from the EBL, WBT, and NBT.

- The SBT and NBL movements possibly need capacity improvements, as will be discussed shortly.

NBL Travel Time

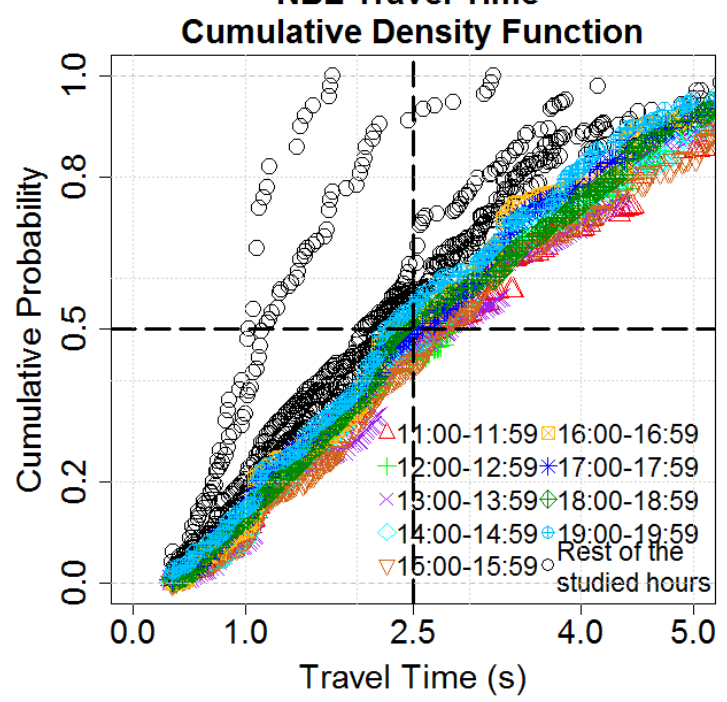

NBT Travel Time Cumulative Density Function

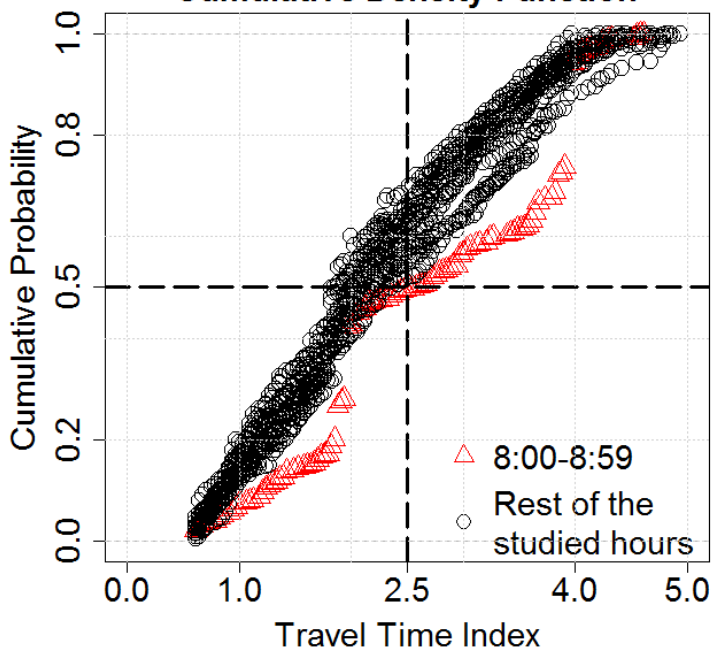

Figure 3-23 Cumulative Density Functions by Hours for Northbound Movements.

In addition, it seems that the timing plan between 3:00 PM and 3:59 PM is unable to accommodate the mainline traffic, thus switching to the peak period plan is recommended. To explore this further, the historical Time-of-Day (TOD) signal timing schedule at the target intersection is presented in Figure 3-25. The intersection has separate timing plans between 3:00 PM to 3:59 PM (i.e., Plan 11) during weekdays, which assigns shorter green times to the EBT and WBT movements, compared to the PM peak (i.e., 4:00 
PM to 6:59 PM). This confirms the need to either apply Plan 12 starting at 3:00 PM, or fine-tune Plan 11 to give more green time to the main street thru movements.
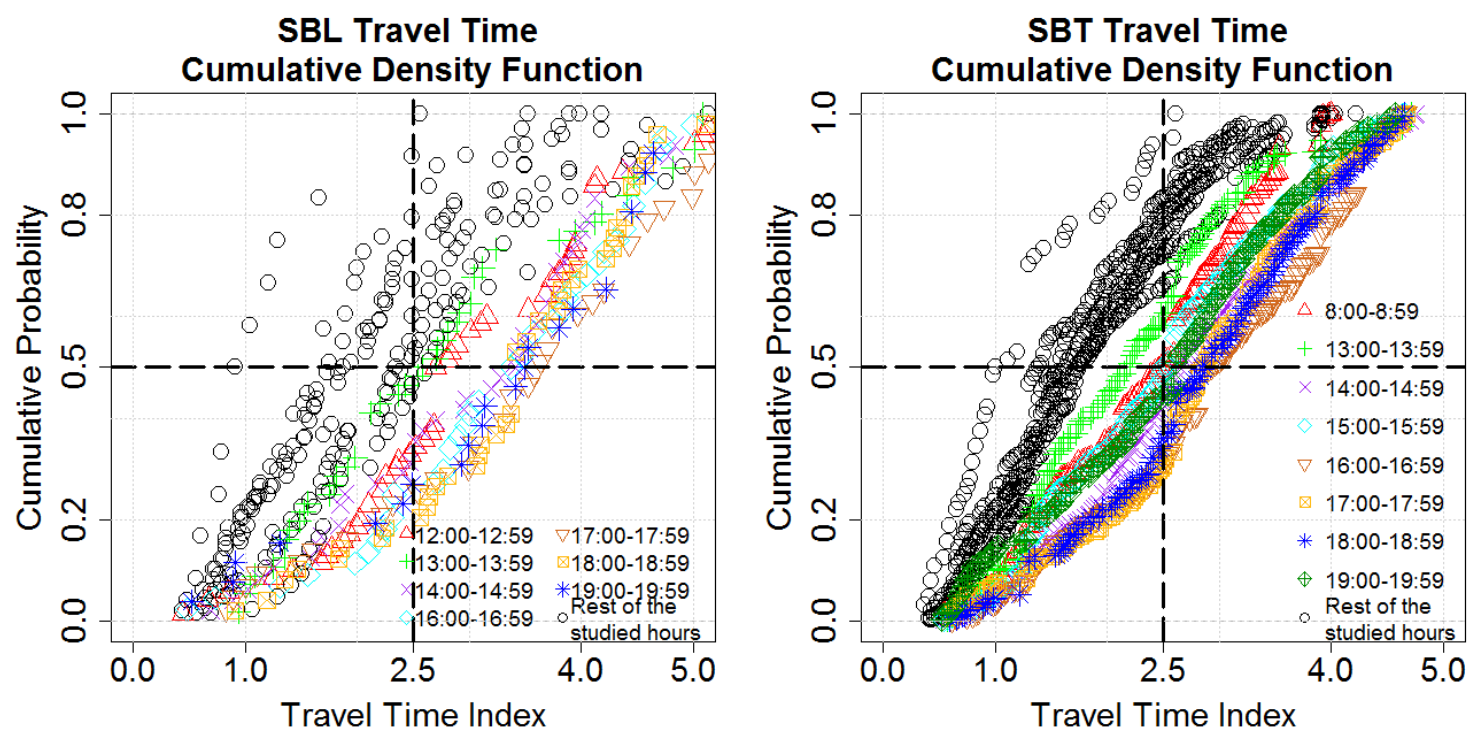

Figure 3-24 Cumulative Density Functions by Hours for Southbound Movements.

Figure 3-25 also indicates that the SBL movement receives a small portion of the green time in the cycle, compared to other movements. The CDF plots show the deterioration in travel time performance of the SBL movement after 12:00 PM, and the field observations reported heavy traffic congestion at that time of day. A possible increase of the green time of Phase 3 for the SBL movement should help improve its TTI performance. In contrast, the SBT movement receives a large portion of green time but still shows poor TTI performance, indicating a possible need for capacity increase. It is worth mentioning that the phases for the northbound and southbound directions are noncoordinated. That also contributes to the performance issues with the southbound movements. In the NBL movement, the TTI performance is found to be inferior during most times of the studied period. This indicates that the NBL movement either needs more green time or a geometry improvement. 


\begin{tabular}{|c|c|c|c|c|c|c|c|c|c|c|c|c|}
\hline \multicolumn{13}{|c|}{ Green Time } \\
\hline$\frac{\text { Current }}{\text { TOD Schedule }}$ & Plan & Cycle & $\begin{array}{c}1 \\
\mathrm{EBL}\end{array}$ & $\begin{array}{c}2 \\
\text { WBT }\end{array}$ & $\begin{array}{c}3 \\
\text { SBL }\end{array}$ & $\begin{array}{c}4 \\
\text { NBT }\end{array}$ & $\begin{array}{c}5 \\
\text { WBL }\end{array}$ & $\begin{array}{c}6 \\
\text { EBT }\end{array}$ & $\begin{array}{c}7 \\
\text { NBL }\end{array}$ & $\begin{array}{c}8 \\
\text { SBT }\end{array}$ & Ring Offset & Offset \\
\hline & Free & & & & & & & & & & & \\
\hline$\underline{0100}$ & Free & & & & & & & & & & & \\
\hline$\underline{0500}$ & 17 & 120 & 14 & 35 & 8 & 37 & 15 & 34 & 8 & 37 & 0 & 13 \\
\hline$\underline{0530}$ & 2 & 140 & 14 & 54 & 9 & 37 & 13 & 55 & 9 & 37 & 0 & 104 \\
\hline$\underline{0630}$ & 4 & 190 & 28 & 55 & 12 & 69 & 19 & 64 & 23 & 58 & 0 & 56 \\
\hline$\underline{0930}$ & 7 & 150 & 18 & 38 & 12 & 56 & 18 & 38 & 12 & 56 & 0 & 45 \\
\hline 1400 & 9 & 160 & 19 & 43 & 15 & 57 & 19 & 43 & 21 & 51 & 0 & 150 \\
\hline 1500 & 11 & 170 & 18 & 40 & 13 & 73 & 21 & 37 & 22 & 64 & 0 & 103 \\
\hline 1600 & 12 & 190 & 19 & 51 & 13 & 81 & 16 & 54 & 25 & 69 & 0 & 103 \\
\hline 1900 & 13 & 170 & 23 & 40 & 11 & 70 & 23 & 40 & 18 & 63 & 0 & 84 \\
\hline$\underline{2030}$ & 14 & 150 & 20 & 34 & 12 & 58 & 20 & 34 & 12 & 58 & 0 & 140 \\
\hline$\underline{2130}$ & 15 & 130 & 16 & 39 & 7 & 42 & 15 & 40 & 9 & 40 & 0 & 9 \\
\hline 2230 & 16 & 120 & 14 & 37 & 7 & 36 & 14 & 37 & 7 & 36 & 0 & 9 \\
\hline
\end{tabular}

Figure 3-25 Time-of-Day Signal Timing Plan Schedule Report for the Intersection of Southwest 8th Street at Southwest 107th Avenue

This section presents a methodology on utilizing TTI and aggregating them on the CDF plots to investigate traffic conditions. However, the interpretation of the CDF plots relies on human experience. Moreover, it only utilizes traffic conditions on signal operation diagnosis. It lacks information on the traffic signal itself and therefore does not have a full picture of the signal operation in the field. In sum, using CDF plots alone is not enough for automatic signal operation diagnosis. An additional attempt is made to automate the process of identifying the need for improvements by combining interpretations from the CDF plots above with other measures such as the signal green time max-outs for various movements. A description of this effort is presented in the later section.

\subsection{Signal Data Preprocessing}

The above analysis is based on Wi-Fi data analyses that provide information about vehicle travel times. However, utilizing only Wi-Fi data may lead to biased results on signal diagnosis since it does not utilize any information from the traffic signal operations. In order to better understand the signal impacts on traffic conditions, which are indicated by the TTI from Wi-Fi data as described earlier, information of the traffic signal operation can 
be obtained from the historical signal timing records downloaded from the central signal control software. A signal timing performance measure, the Max-out Ratio $(M R)$, is introduced herein to represent the traffic signal performance. This parameter is calculated from Equation 3-5 listed below, in which the values of the variables can be extracted from the historical signal timing records.

$$
M R=\frac{\text { Number of Max-outs }}{\text { Number of Cycles }}
$$

As can be seen from the equation above, $M R$ is calculated as the ratio of the number of times a phase experiences max-out to the total number of cycles in the studied time period. According to the signal timing manual (Koonce et al. 2008), max-out is defined as

"a type of actuated operation for a given phase where the phase terminates due to reaching the designated maximum green time for the phase." Thus, if a phase experiences a maxout, it indicates that the demand for green time of that phase exceeds the allocated green time in the corresponding cycle. In other words, the $M R$ represents the frequency in which the green time demand of a phase exceeds its preset value.

\subsection{Signal Operation Diagnosis}

\subsubsection{Diagnostic Scheme}

Traffic conditions were derived from the CDF plots of $T T I$ that were aggregated from travel time information. Although CDF plots provide excellent visualization of TTI performance, the conclusions were made based on human interpretation of the CDF plots. On the other hand, combining the preprocessed Wi-Fi data and signal data, the relationship between signal control parameters and vehicle travel times can be identified, allowing even better support for signal operation diagnosis. A diagnostic scheme is proposed in this context and 
presented in Figure 3-26. The proposed scheme diagnoses the green time allocation for the individual signal phase first. It then divides phases into various sections and diagnoses the sectional green time allocation. Finally, the scheme inspects the cycle length or capacity adequacy for the entire intersection based on the results from the previous steps.

As is the case with the CDF analyses presented in the previous section, TTI is used as the performance measure for the evaluation of traffic conditions. The TTI used in the proposed diagnostic scheme is calculated from the Wi-Fi detections as the ratio of the actual travel time to the estimated free-flow travel time. As discussed earlier, the $15^{\text {th }}$ percentile travel time of all weekday data is used as the estimated free-flow travel time for each movement. The $50^{\text {th }}$ percentile $T T I$ is used as the measure that represents travel time in this dissertation. However, the analyst may replace the $50^{\text {th }}$ percentile $T T I$ with the $80^{\text {th }}$ percentile TTI or any other TTI. Similarly, the users of the proposed diagnostic scheme can change the values of the estimated free-flow travel time. Selecting different percentile TTI in the analysis would reflect an agency's desire to make its decision based on the worst $20^{\text {th }}$ percentile of traffic (i.e., if the $80^{\text {th }}$ percentile $T T I$ is used) versus the worst $50^{\text {th }}$ percentile of traffic (i.e., if the $50^{\text {th }}$ percentile $T T I$ is used). A utility index that combines different percentile $T T I$ can also be used. 


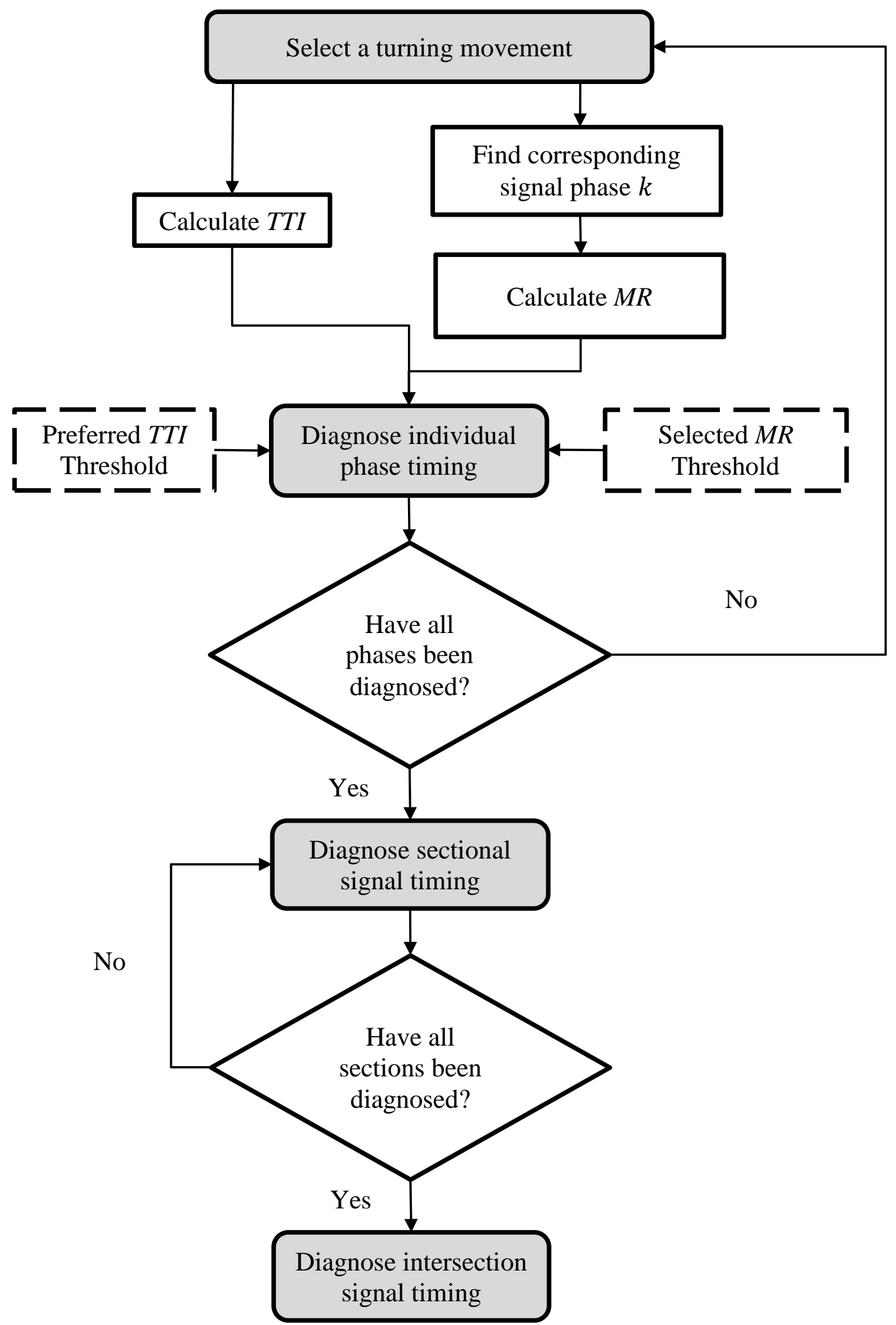

Figure 3-26 Proposed Decision-Support Signal Operation Diagnostic Scheme 
In this dissertation, a typical dual-ring eight-phase coordinated signal control is investigated in the framework development because it is the type of signal control utilized at the studied intersection. However, the proposed framework can be applied to any other type of fixed-time or actuated signal control. As shown in Figure 3-26, the proposed scheme first inspects the performance of the individual phase for the corresponding turning movement. At the individual phase diagnosis stage, the $T T I$ and $M R$ are compared to selected thresholds. The comparison results are then combined to examine whether or not the allocated green times for the phases are appropriate. For example, if a signal phase experiences high $T T I$ and high $M R$, the diagnosis will recommend increasing the preset green time for that individual phase. The rules of the diagnosis will be presented shortly.

After diagnosing all individual phases, a sectional level diagnosis is conducted by grouping specific individual phases together. An intersection level diagnosis is then performed on the basis of the results from the previous diagnoses. The intersection level diagnosis inspects whether the cycle length can provide sufficient capacity to serve the overall intersection in order to avoid oversaturation. At this intersection level signal diagnosis stage, the inspection considers all diagnosis results of the individual phase, and the sections combine them. It then searches for opportunities to switch green times between phases, if there are any. The diagnostic scheme also takes the signal control logic into account and examines the feasibility of the green time exchange between phases.

\subsubsection{Individual Phase Diagnosis}

The studied intersection is controlled by a typical dual-ring eight-phase coordinated signal control; the phase diagram is illustrated in Figure 3-27. With this control, Phase 2 and 
Phase 6, which corresponds with the EBT and WBT movement for the studied intersection, are the coordinated phases. The coordinated phases are always given green time that are equal to or are more than the preset maximum value, regardless of traffic conditions, and thus the max-out information is not used in the analysis of these phases. On the other hand, the uncoordinated phases will have a gap-out if there are not enough demands on the corresponding movements. Consequently, the diagnostic scheme only combines TTI and $M R$ to diagnose the uncoordinated phases and uses $T T I$ as the only measures for coordinated signal phase diagnosis.

\begin{tabular}{|c|c|c|c|}
\hline Phase 1 & Phase 2 & Phase 3 & Phase 4 \\
Uncoordinated & Coordinated & Uncoordinated & Uncoordinated \\
\hline Phase 5 & Phase 6 & Phase 7 & $\begin{array}{c}\text { Phase 8 } \\
\text { Uncoordinated }\end{array}$ \\
\hline
\end{tabular}

Figure 3-27 Typical Dual-Ring-Eight-Phase Signal Diagram for the Signal Control at the Studied Intersection

The uncoordinated signal phases (and their corresponding movements) in this case include Phase 1 (EBL), Phase 3 (SBL), Phase 4 (NBT), Phase 5 (WBL), Phase 7 (NBL), and Phase 8 (SBT). In order to diagnose whether or not the allocated green times are adequate for the traffic conditions, the user-selected $T T I$ and $M R$ thresholds are compared with the corresponding measures derived from the field data. For an uncoordinated phase $k$ at a time period of the day, recommendations are given based on the following criteria:

- If $T T I_{k} \leq T T I_{T H, k}$ and $M R_{k} \leq M R_{T H, k}$, then $D P_{k}=-1$; 
- -1 signifies the potential to reduce the green time of the phase;

- If $T T I_{k} \leq T T I_{T H, k}$ and $M R_{k}>M R_{T H, k}$, then $D P_{k}=0$;

○ 0 signifies no recommendations regarding reducing or increasing the green time of the phase;

- If $T T I_{k}>T T I_{T H, k}$ and $M R_{k} \leq M R_{T H, k}$, then $D P_{k}=0$;

- If $T T I_{k}>T T I_{T H, k}$ and $M R_{k}>M R_{T H, k}$, then $D P_{k}=1$.

- 1 signifies the need to increase the green time of the phase

where

$T T I_{k}=\quad$ the field-estimated TTI for phase $k$. In this case, phase $k$ is an uncoordinated phase.

$T T I_{T H, k}=\quad$ the user-selected TTI threshold for phase $k$.

$M R_{k}=\quad$ the measured max-out ratio for phase $k$.

$M R_{T H, k}=\quad$ the user-selected $M R$ threshold for phase $k$.

$D P_{k}=\quad$ the diagnosis result for phase $k$.

The above diagnoses and recommendations are summarized in Table 3-3.

Table 3-3 Diagnoses of the Uncoordinated Phases

\begin{tabular}{|c|c|c|c|}
\hline $\begin{array}{c}\text { calculated } T T I \text { vs. TTI } \\
\text { threshold }\end{array}$ & $\begin{array}{c}\text { calculated } M R \text { vs. } M R \\
\text { threshold }\end{array}$ & $\begin{array}{c}\text { recommendation on } \\
\text { green time }\end{array}$ & code \\
\hline$\leq$ & $\leq$ & decrease & -1 \\
\hline$\leq$ & $>$ & keep & 0 \\
\hline$>$ & $\leq$ & keep & 0 \\
\hline$>$ & $>$ & increase & 1 \\
\hline
\end{tabular}

As discussed earlier, the examination of TTI provides information on the movement operation conditions, and the examination of an $M R$ reflects the green time demand of the 
corresponding signal phase. Therefore, the above criteria for an individual signal phase essentially attempts to answer the following questions:

1. Is the movement congested?

2. Is the assigned green time adequate?

For example, the first criterion in Table 3-3 indicates that a movement is not congested and the assigned green time is more than adequate. In this case, the corresponding phase is flagged to be the candidate that will potentially decrease the green time. When a movement has low TTI but experiences frequent max-outs (i.e., high $M R$ ), it implies that the assigned green time setting is well utilized. As a result, the diagnosis recommends keeping the current setting. It is noteworthy that as described by the third criterion, when the movement suffers from high TTI but the allocated green time seems to be adequate (i.e., low $M R$ ), the reason for the high $T T I$ is uncertain and should be further examined. The reasons may be due to conditions such as signal settings or geometric conditions leading to frequent gap-outs, even though the demand of the corresponding movement is high. It can also occur when the corresponding movement is blocked by another movement on the same approach but controlled by a different phase (such as a leftturn movement blocked by a through movement). With these conditions, there is no evidence to support changing the phase timing. The diagnosis therefore recommends keeping the current setting. Lastly, if a movement experiences high TTI and high max-out frequency, this indicates that there is a demand for more green time for the phase that serves the movement.

Since the coordinated phases assigned green times that are equal to or are more than the maximum green time, utilizing the $M R$ in the diagnostic scheme for the coordinated 
phases is meaningless. Therefore, the criteria for diagnosing the assigned green time on a coordinated phase $k$ is only based on TTI, as presented below.

- If $T T I_{k}<T T I_{T H, k}$, then $D P_{k}=-1$;

- If $T T I_{k}=T T I_{T H, k}$, then $D P_{k}=0$;

- If $T T I_{k}>T T I_{T H, k}$, then $D P_{k}=1$.

The diagnoses and recommendations are summarized in Table 3-4.

\section{Table 3-4 Diagnoses of Coordinated Phases}

\begin{tabular}{|c|c|c|}
\hline calculated TTI vs. TTI threshold & recommendation on green time & code \\
\hline$<$ & decrease & -1 \\
\hline$=$ & keep & 0 \\
\hline$>$ & increase & 1 \\
\hline
\end{tabular}

\subsubsection{Sectional Level Diagnosis}

Since the settings of the dual-ring controller result in dependency between phases, recommendations to change signal phase timing will have to acknowledge these dependencies. For example, if the EBL green is excessive but the WBT traffic is heavy, then it is possible to switch the green time from the EBL to the WBT. In contrast, if the EBL green time is excessive but the EBT needs more, the green time cannot be transferred from the EBL to the EBT.

Numeric categorical variables are continually used to represent various diagnosis results. Based on the above discussion, " 1 " is used to represent the recommendation to increase the green time, "0" indicates the recommendation to keep the allocated green time, and "- 1 " stands for the recommendation to flag the phase for potential green time decrease. The recommendations are stored for later diagnosis on the signal plan of the whole intersection. As can be seen from the diagnosis scheme in Figure 3-26, after diagnosing 
individual phases, a judgement is placed to see whether all phases have been diagnosed. If there are still phases that have not been diagnosed, the system will go back to the phase selection and start the diagnosis on an individual phase again. The diagnostic scheme for the intersection level signal timing is based on all individual phase diagnosis results. Therefore, unless all phases are diagnosed, the scheme will not move on to the next level of diagnosis.

Once all individual phase diagnoses are completed, the system will start the sectional level diagnosis by first dividing the eight signal phases into four sections. As shown in Figure 3-28, the sections are naturally divided by the barrier (i.e., the vertical solid line) and the ring boundary (i.e., the horizontal dashed line). Hence, the sections of the dual-ring eight-phase signal control can be divided as:

- Section 1: ring 1 and barrier 1

- Section 2: ring 1 and barrier 2

- Section 3: ring 2 and barrier 1

- $\quad$ Section 4: ring 2 and barrier 2

Phases inside each divided section can exchange green time with each other without affecting the phase timing in other sections. For instance, Phase 2 can borrow some green time from Phase 1 without violating either the barrier or the ring boundary, but other phases cannot take any green time from Phase 1 freely since they are separated by either the ring boundary or the barrier.

A sectional phase diagnostic scheme is then applied to each section to determine whether there is a need to increase the green time for that section. Each section has two phases in the dual-ring eight-phase signal control, and each phase will have three possible 
diagnosis results, as discussed earlier. There will be a total of nine (i.e., $3^{2}=9$ ) combinations of individual phase diagnosis results in each section. Using the numeric categorical variables, the diagnosis results of each section based on the combinations of individual phase recommendations are shown in Figure 3-29.

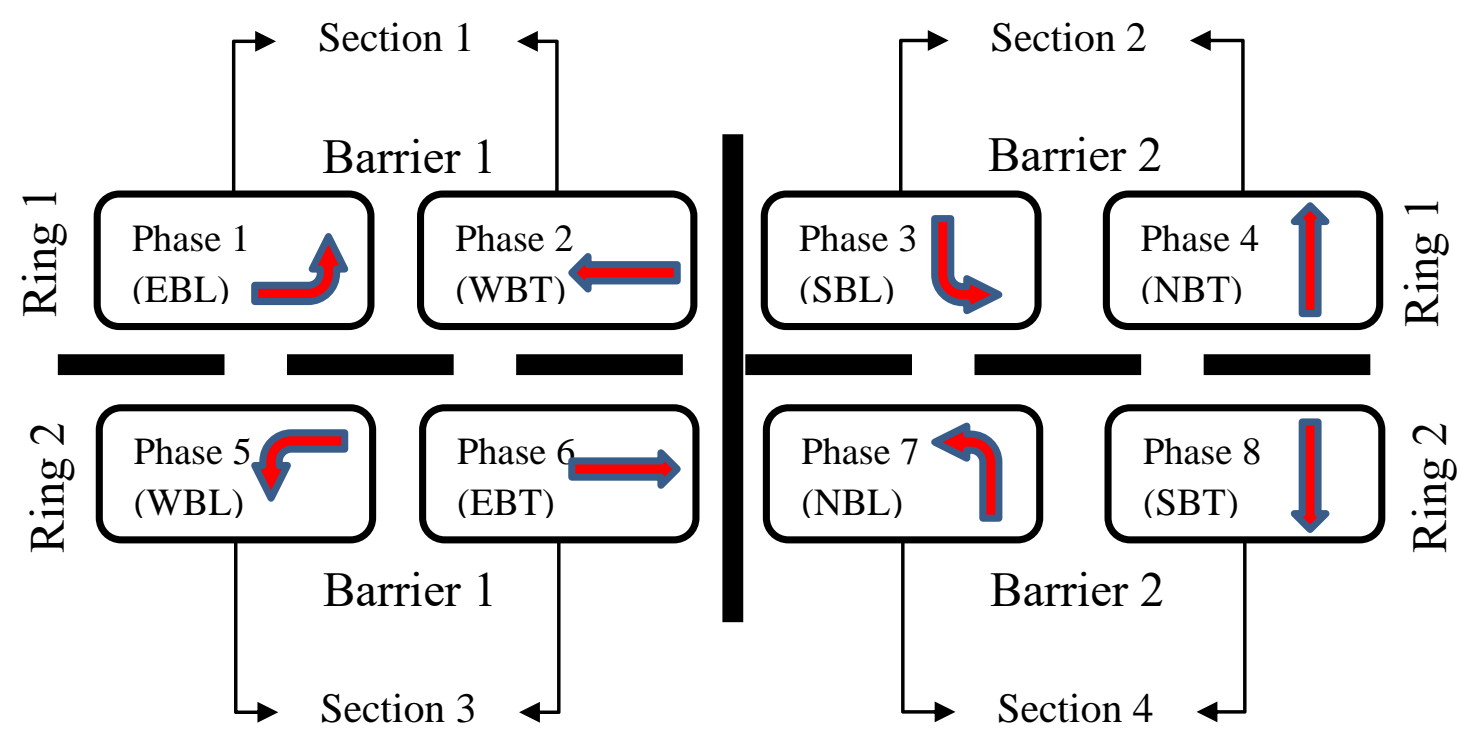

Figure 3-28 Illustration of Signal Phase Sections in the Phase Diagram

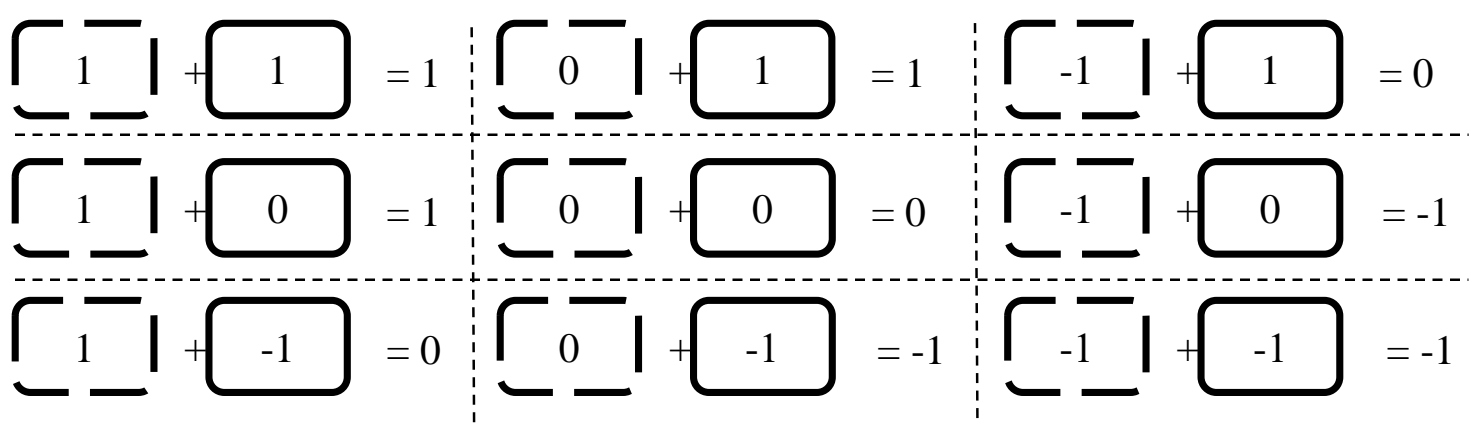

Where,

$\begin{array}{ll}1 / 0 /-1 & \begin{array}{c}\text { diagnosis of the leading } \\ \text { phase in the section. }\end{array} \quad 1 / 0 /-1 \quad \begin{array}{c}\text { diagnosis of the lagging } \\ \text { phase in the section. }\end{array}\end{array}$

Figure 3-29 Sectional Diagnostic Scheme

As shown in Figure 3-29, the diagnosis results can be described as follows: 
- In each section, the total allocated green time of the two phases in the section is recommended to increase if:

$\circ$ there is at least one phase that needs more green time (" 1 "); and

$\circ$ the green time of the other phase is not flagged to be a candidate of green time decrease (“" 0 ” or " 1 ”).

- The diagnosis recommends flagging the section as a candidate of the sectional green time decrease if:

○ at least one phase shows an interest in decreasing the green time ("-1"); and

○ the other phase is not recommended to increase its green time (" 0 " or "- 1 ").

- The diagnosis recommends keeping the sectional green time for the remaining combinations of individual signal diagnosis results. More specifically, the total allocated sectional green time should be kept if:

○ both phases are recommended to keep their green time (“ 0 ”); or

- one phase is recommended to increase its green time (" 1 "); and

- the other is flagged as a candidate to have its green time decreased $("-1 ")$.

It should be noted that if one phase is recommended to increase the green time (" 1 ") and the other is recommended to do the opposite (“-1"), then the system suggests keeping the current setting due to the lack of evidence to suggest any changes, and a further investigation is needed. In spite of the fact that the green time can be exchanged between the two phases in a section, there may be still a need to increase the total allocated green time. In addition, it is interesting to note that the rules can be summed up mathematically, as presented below. 
- If $P_{i, 1}+P_{i, 2}>0$, then $D S_{i}=1$;

- If $P_{i, 1}+P_{i, 2}=0$, then $D S_{i}=0$;

- If $P_{i, 1}+P_{i, 2}<0$, then $D S_{i}=-1$.

where

$P_{i, 1}=$ the leading phase in section $i$.

$P_{i, 2}=$ the lagging phase in section $i$.

$D S_{i}=$ represents the diagnosis result of the total allocated green time for section $i$.

\subsubsection{Intersection Level Diagnosis}

With the completion of the individual phase diagnoses and sectional level diagnoses, the signal plan for the entire intersection, in particular, the cycle length, is evaluated based on the previous results. The intersection level diagnosis, which is similar to the presentation of the sectional level diagnosis, is shown in Figure 3-30 and Figure 3-31. Figure 3-30 shows all of the combinations resulting in "1" as the diagnosis results for the cycle length. Similar to the definition of the numeric categorical variables used earlier, the diagnosis result of " 1 " indicates the need for a longer cycle length. More specifically, increasing the capacity

of the entire intersection is required if the criteria are to be met. For the signal plans that already have long cycle lengths, geometrical improvement may be required.

As can be seen in Figure 3-30, the intersection level diagnosis decides whether to recommend increasing the cycle length by examining the diagnosis results from two sections located in different barriers. It is recommended to increase the cycle length if the sum of the diagnosis results from each pair of two sections is positive. The diagnoses can 
be described mathematically as the following. Each rule is based on one row in Figure 3-30, which contains three graphs.

- If $D S_{1}+D S_{2}>0$, then $D I=1$;

- If $D S_{3}+D S_{4}>0$, then $D I=1$;

- If $D S_{1}+D S_{4}>0$, then $D I=1$;

- If $D S_{2}+D S_{3}>0$, then $D I=1$.

where

$D I=$ denotes the diagnosis result of the intersection level diagnosis.

It is worth pointing out that any of the above four conditions will lead to the intersection level diagnosis of " 1 ," requesting a cycle length increase for the whole intersection. Each of the conditions only examines the diagnosis results of two sections. Therefore, the remaining two sections can have any diagnosis results, and the intersection level diagnosis will be the same. However, no diagnosis results are given if only the two sectional results in the same barrier (i.e., Section 1 and Section 3, Section 2 and Section 4) are examined. Since the sections in the same barrier may exchange green time with the sections in the other barrier, further investigation is needed. Each section with a letter "A" in Figure 3-30 can have three sectional diagnosis results. As a result, the intersection level diagnosis of "1" can process $9 \times 3+6 \times 3+4 \times 3+2 \times 3=63$ combinations, given a total of $3^{4}=81$ scenarios.

Figure 3-31 shows the opposite when the diagnostic result for the intersection level signal timing is " -1 ," meaning that the cycle length of the intersection can be reduced. As can be seen from the figure, no diagnosis result of " 1 " is found in all sections. In addition, there are at least two sections diagnosed as "- 1. ." In either barrier, both sections in the two 
rings should have the sectional level diagnosis result of "-1" (e.g., Section 1 and Section 3, or Section 2 and Section 4). The conditions can be summarized mathematically, as presented below.

- If $D S_{1}<0$, and $D S_{3}<0$, and $D S_{2} \leq 0$, and $D S_{4} \leq 0$, then $D I=-1$;

- If $D S_{2}<0$, and $D S_{4}<0$, and $D S_{1} \leq 0$, and $D S_{3} \leq 0$, then $D I=-1$.

It is interesting to point out that the second rule is the same as the first rule but is only applied to the different sides of the barrier. As seen in the figure, the intersection level diagnosis of "-1" can handle 7 out of 81 combinations of the sectional level diagnosis results.

The scenarios shown in Figure 3-30 and Figure 3-31 recommend changing the cycle length, with $63+7=70$ combinations of the sectional level diagnosis results. Therefore, the remaining $81-70=11$ scenarios will result in the intersection level diagnosis of " 0 ," yielding a recommendation to keep the cycle length setting as long as it is appropriate or there is not enough evidence to support the change.

It seems it is easier to achieve the criteria of increasing cycle length, compared to keeping or decreasing it. It is reasonable because of the nature of the signal control logic. An at-grade intersection has more pairs of conflicting movements than overlapping movements. In this context, it is difficult to exchange green time between signal phases since there is a high chance that they will conflict with each other. Given the fact that an increasing cycle length is an outcome of a failure in the exchange of green time between phases, qualifying the cycle length decrease is the most difficult part; it is relatively easier to qualify increasing it. 


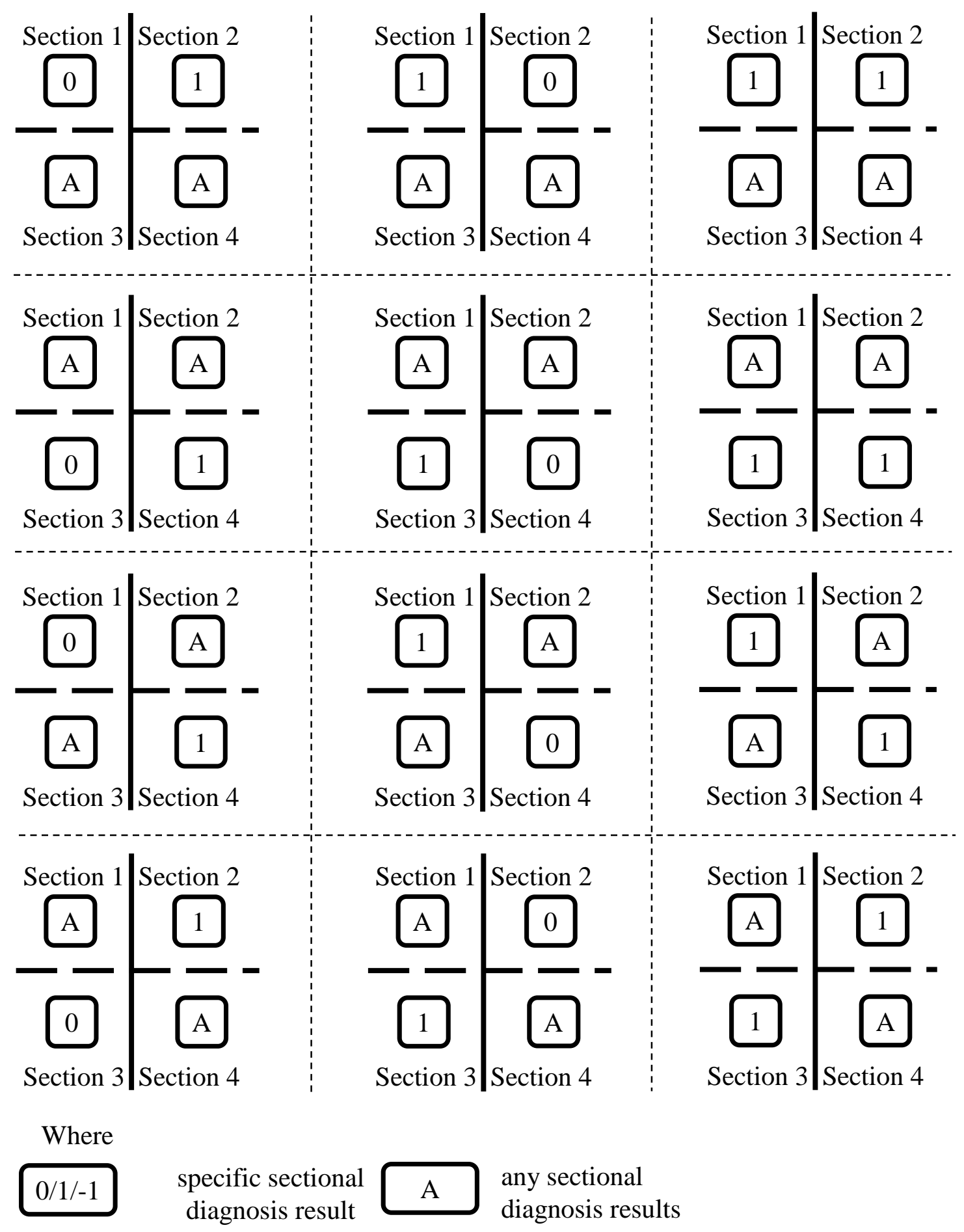

Figure 3-30 Combinations While Intersection Level Diagnosis Results are "1" 


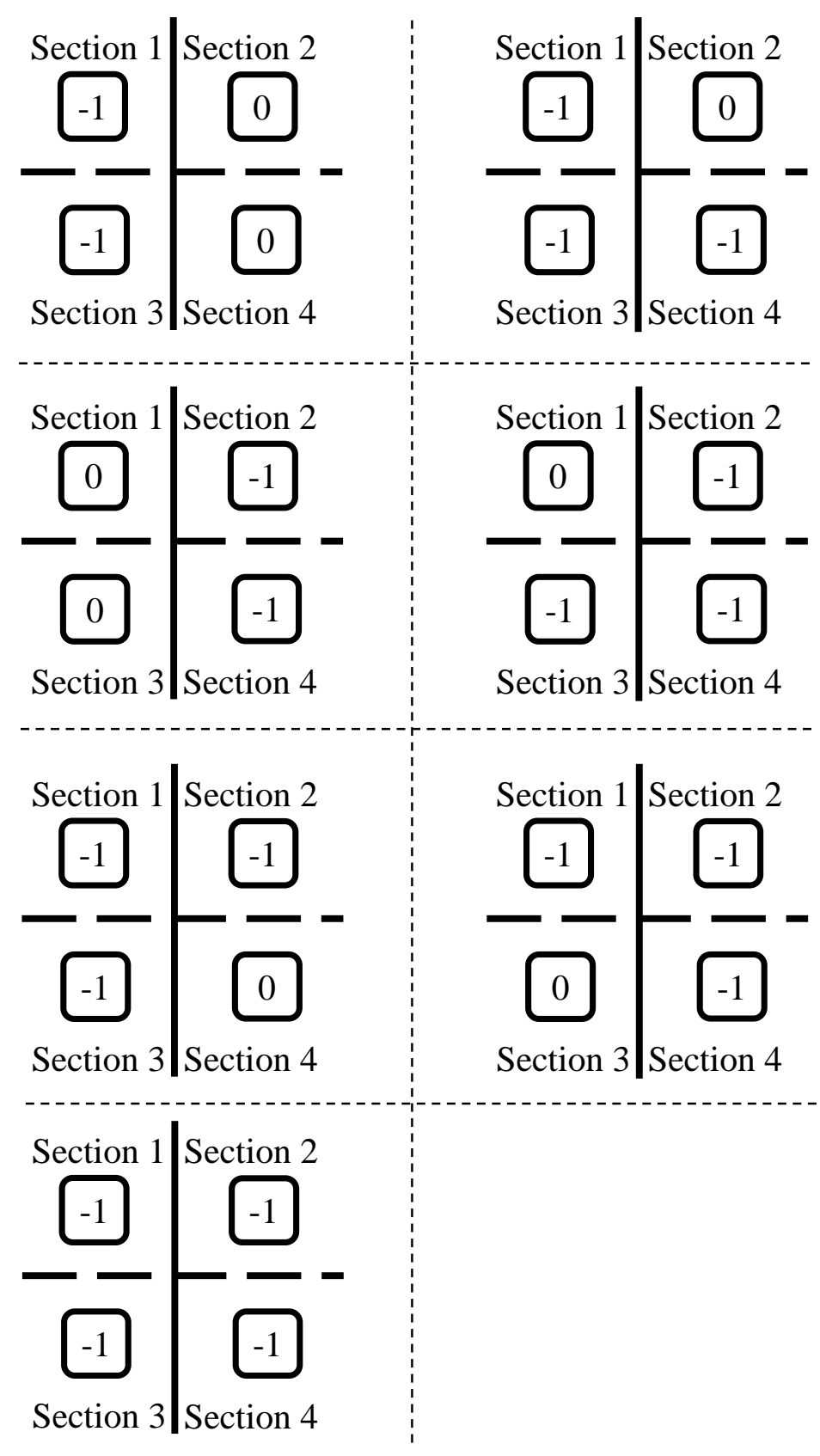

Where

specific sectional
diagnosis result

Figure 3-31 Combinations While Intersection Level Diagnosis Results are "-1" 


\subsection{Summary}

In this chapter, the methodology framework of this dissertation is presented. With the appropriate installation of the Wi-Fi readers, the devices traveled between the studied intersection and the surrounding intersections can be matched. The methodology of data matching is applied in order to avoid mismatches, as well as to identify multiple trips made by each device. By matching the identified trips between the studied intersection and its surrounding intersections, turning movements can be categorized based on the matches of MAC addresses and the corresponding timestamps between different trips.

Various pairs of timestamps between the studied intersection and its upstream intersections at different detection stages are discussed in this chapter. The last-to-last travel times are selected in the calculation of trip travel times between the studied intersection and its upstream intersections. The calculated travel times are then used to distinguish traversals from all identified trips by applying the presumed shortest travel times and DBSCAN algorithm in various turning movements.

The travel times of the valid traversals are then aggregated to investigate the traffic conditions in the field. TTI is used as a performance measure, and CDF plots of TTI are utilized to represent the traffic status during different times of day. Moreover, information extracted from the historical signal timing records of the studied intersection signal control are combined with the $\mathrm{CDF}$ plots in order to obtain accurate information from the field traffic conditions and signal performance.

Since CDF plots rely on human interpretation, an automatic diagnosis scheme is developed, which utilizes $T T I$ derived from the Wi-Fi readings and $M R$ calculated from the signal records. TTI sheds light on the traffic conditions, and $M R$ reflects the signal 
operations. The combination of the two measures provides a complete picture of signal performance at the studied intersection.

The scheme first diagnoses the individual phase green time allocation. By dividing individual phases into different sections based on the location in the ring-barrier diagram, the scheme then diagnoses the total allocated green time for each section. Lastly, the scheme diagnoses the cycle length of the intersection based on the previous sectional level diagnosis results. Combining the results from different level diagnoses, the developed framework of signal operation diagnosis is able to provide information to decision-makers on the needs of signal timing improvements, as well as the direction of the suggested improvements. The implementation and evaluation of the proposed framework of signal operation diagnosis are presented in detail in the next chapter. 


\section{CHAPTER 4}

\section{DIAGNOSIS IMPLEMENTATION}

\subsection{Introduction}

The proposed decision-support framework for traffic signal operation diagnosis was implemented in the studied intersection of Southwest 8th Street and Southwest 107th Avenue in Miami, Florida. The Wi-Fi data and historical signal timing data for all weekdays in December 2015 were utilized. The goal of implementation is to identify issues with the current signal timing plans. Only the data from weekdays were analyzed, based on the fact that weekday traffic conditions are significantly different than the weekends.

The analysis time period is from 5:00 AM to 8:00 PM, and is divided into 30-minute intervals. The framework inspects each interval following the steps illustrated in Figure 3-26. In this implementation, the preferred TTI threshold is set to 2.5 for every turning movement associated with a signal phase. In spite of the fact that the best threshold should be the one which can identify signal operation problems, the selected TTI threshold of 2.5 is widely accepted for urban streets in travel time reliability studies (Zegeer et al. 2014). The preferred $M R$ threshold is set to 0.5 for all uncoordinated signal phases. For each turning movement, the $50^{\text {th }}$ percentile $T T I$ estimated based on the valid traversals is selected to be the measure to assess traffic conditions. The utilization of the $50^{\text {th }}$ percentile $T T I$, which is the median of all TTI for valid traversals, indicates that the diagnosis is based on the conditions of the worst $50 \%$ of traffic. It is noteworthy that the percentile TTI and thresholds can be varied by users to accommodate different situations. The diagnostic 
scheme used in this dissertation is written in the Visual Basic ${ }^{\circledR}$ language and Structured Query Language (SQL).

\subsection{Diagnosis Results}

The proposed scheme of signal operation diagnosis starts with the execution of the individual phase signal timing diagnosis; the results are displayed in Figure 4-1 through Figure 4-8. Figure 4-1 through Figure 4-6 shows the recommendations for the uncoordinated signal phases based on both the TTI and MR. Figure 4-7 and Figure 4-8 display the diagnosis results for the coordinated signal phases based on TTI. As represented by the red dotted line in the figures, the signal diagnosis results of individual phases include the following recommendations:

- "1" recommends to increase phase green time

- "0" recommends to keep the phase green time settings

- “-1" recommends to flag the phase for potential green time decrease

\subsubsection{Diagnosis of Uncoordinated Phases}

As shown in Figure 4-1, the diagnosis indicates that the EBL movement does not need an increase in the green time, and even assigns green time surpluses. The $50^{\text {th }}$ percentile $T T I$ for the EBL movement never exceeds the selected TTI threshold of 2.5. This is consistent with the conclusion drawn from the CDF plots shown in Figure 3-20 and Figure 3-21, indicating that the TTI performance of the EBL is good during most of the studied time periods. Although the TTI deteriorates between 8:00 AM and 8:59 AM, as shown in Figure $3-21$, the $50^{\text {th }}$ percentile $T T I$ is still lower than the threshold at 2.5 , as shown in Figure 4-1. 
The TTI of the NBL movement exceeds the 2.5 threshold in a large proportion of the time periods between 11:00 AM and 4:59 PM, as shown in Figure 4-2. However, the $M R$ does not exceed the 0.5 threshold in most cases. As discussed in Chapter 3, there are numerous reasons for high $T T I$ and low $M R$. It could be due to inappropriate settings of the green extension or the blockage of the NBL movement by the NBT movement. The cause remains uncertain until further investigation, such as field observation, is conducted. The diagnosis suggests keeping the green time setting for the time intervals with high TTI and low $M R$, since there is not enough evidence to support changing it. Nevertheless, it is recommended to increase the green time for the corresponding phase (i.e., Phase 7) at midday for the NBL movement.

The allocated green time for the NBT movement seems to be sufficient to serve the movement since the diagnosis recommended keeping it most of the time, except for a few occasions, as shown in Figure 4-3. As explained earlier, the inferior performance in the timeframe between 8:00 AM and 8:59 $\mathrm{AM}$ is expected to be due to spillback from a downstream traffic control signal. Other than this period, the diagnosis also recommends increasing the green time in the period between 5:30 PM and 5:59 PM, and between 7:00 PM to 7:29 PM, since both $T T I$ and $M R$ slightly exceed the thresholds. However, it should be mentioned that other movements have worse performance than the NBT during the PM period, indicating the need for improvement in geometry, such as an additional lane for the NBL movement, since both the $T T I$ and $M R$ exceed the thresholds.

As can be seen from Figure 4-4, the signal operation seems to be appropriate for the SBL movement in the morning, primarily before 11:29 AM. However, this move 
requires additional green time in the periods that follow. This is consistent with the conclusions drawn from the CDF plots in Figure 3-24.

The diagnosis of the SBT movement, as shown in Figure 4-5, is also consistent with the CDF plots shown in Figure 3-24. The diagnosis indicates the need for additional time between 2:00 PM and 6:59 PM. On the other hand, the diagnosis confirms the CDF interpretations that the green time for the WBL movement is sufficient, as indicated in Figure 4-6. Moreover, Figure 4-6 implies that the signal phase of the WBL movement has extra green time, particularly in the time period before 3:29 PM.

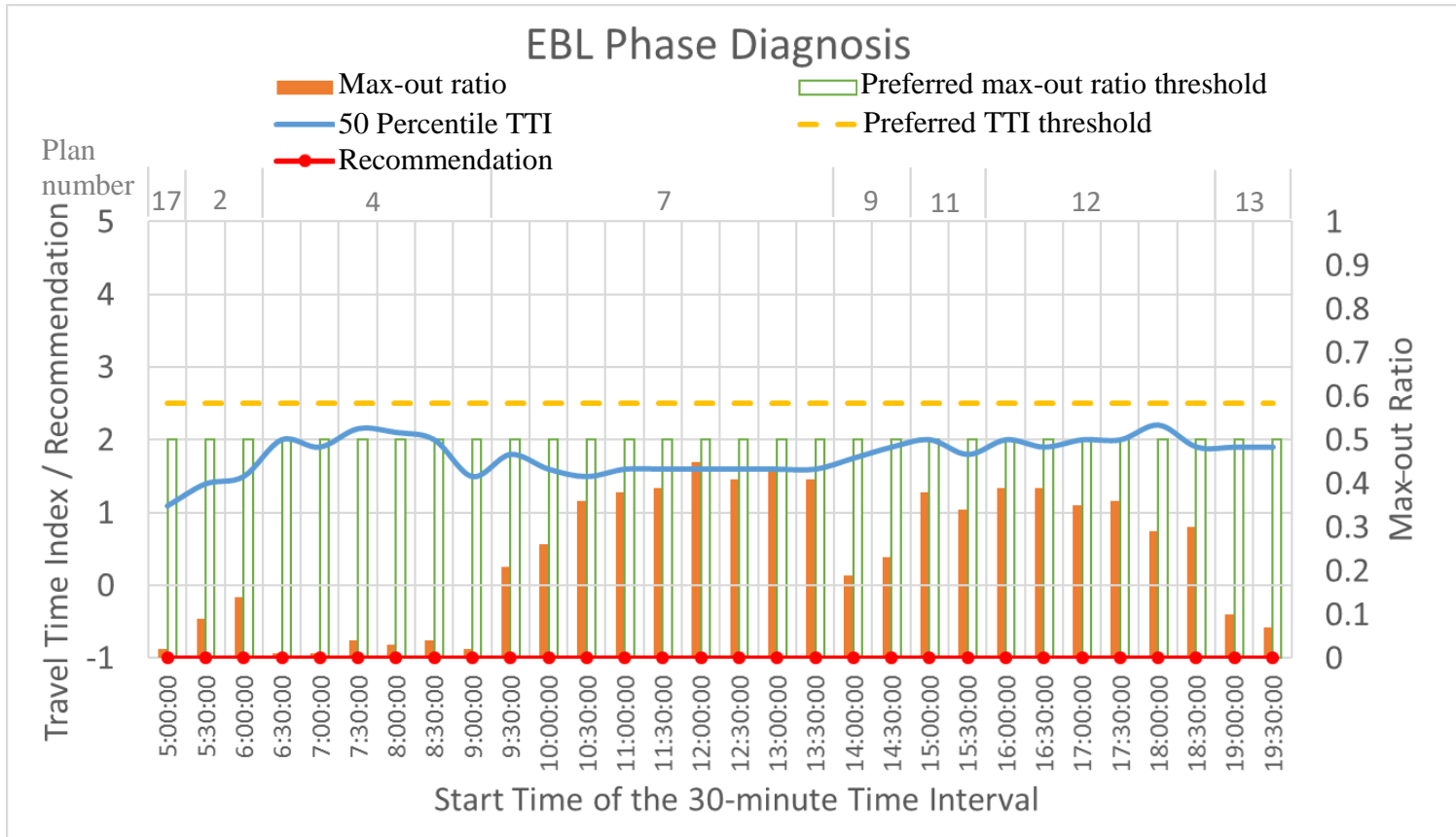

Figure 4-1 Uncoordinated Signal Phase Diagnosis for EBL Movement 


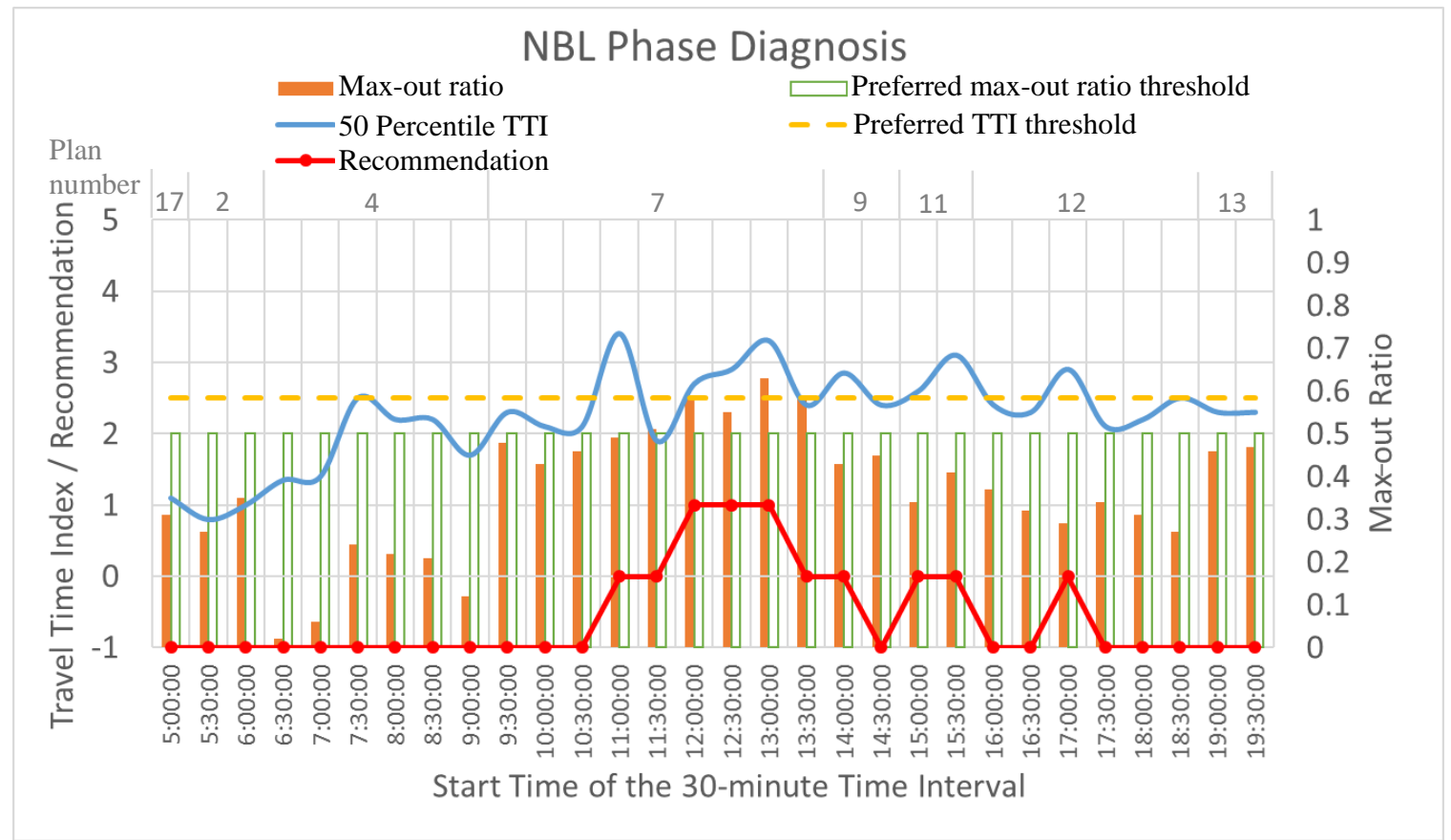

Figure 4-2 Uncoordinated Signal Phase Diagnosis for NBL Movement

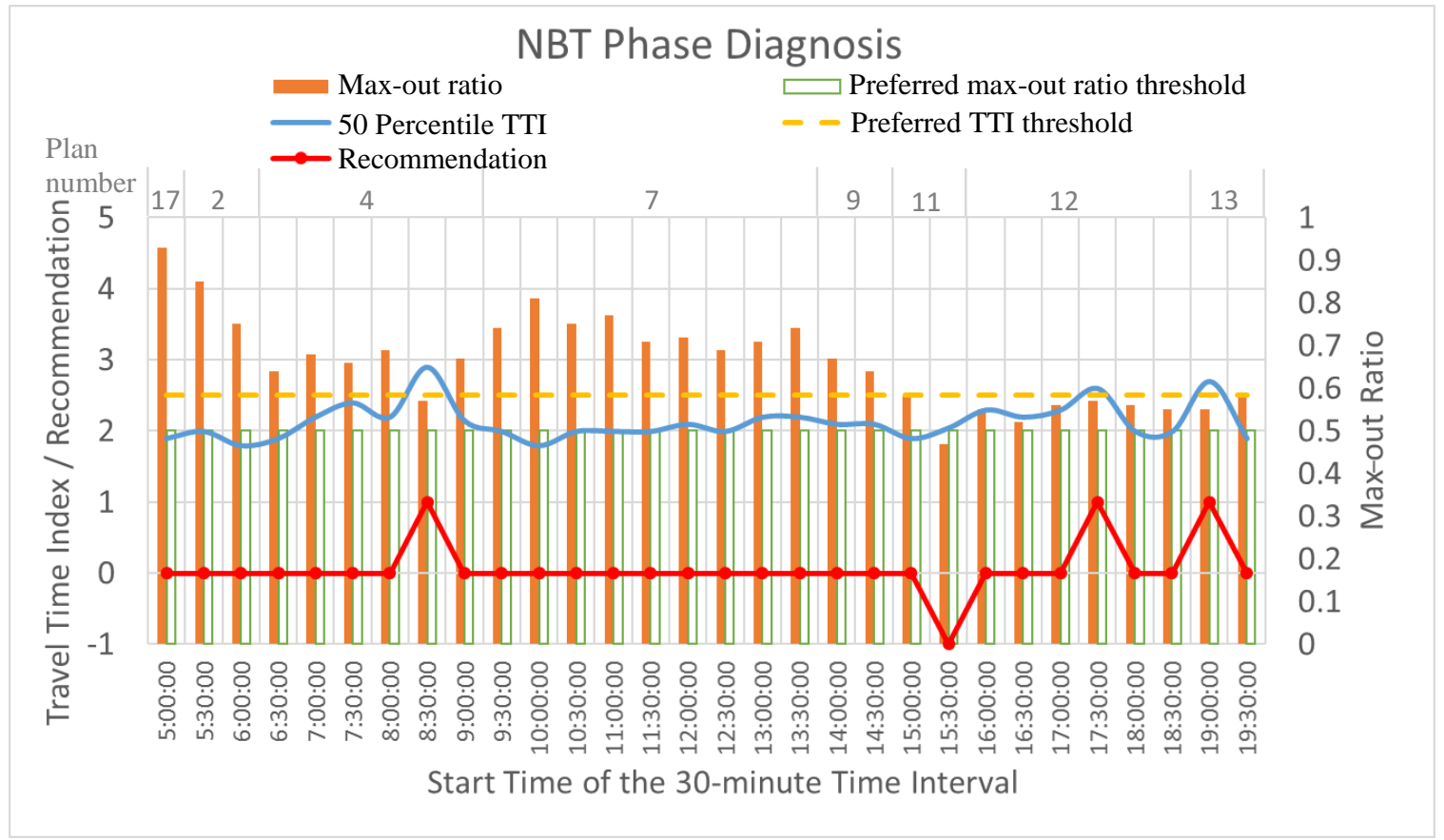

Figure 4-3 Uncoordinated Signal Phase Diagnosis for NBT Movement 


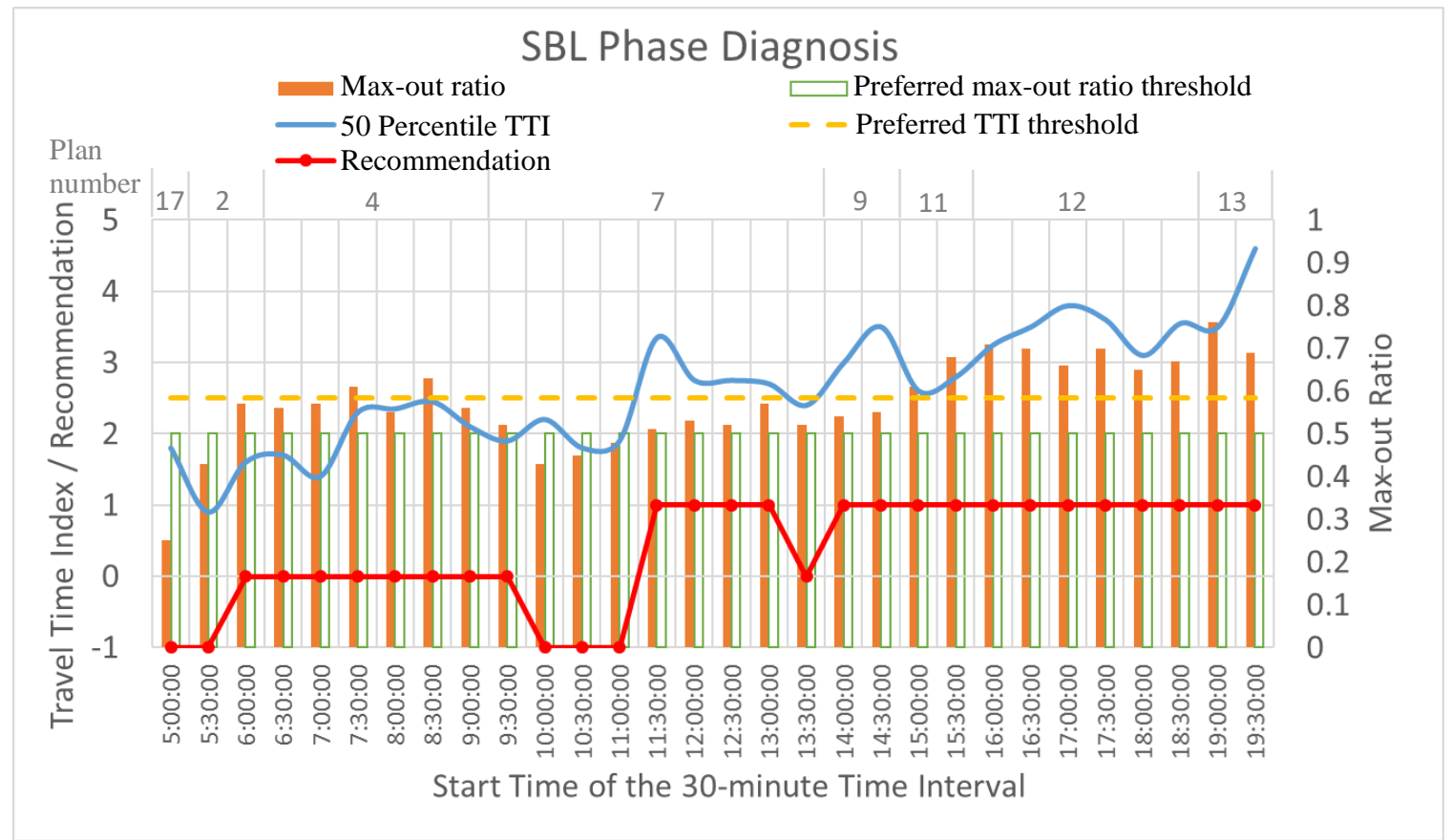

Figure 4-4 Uncoordinated Signal Phase Diagnosis for SBL Movement

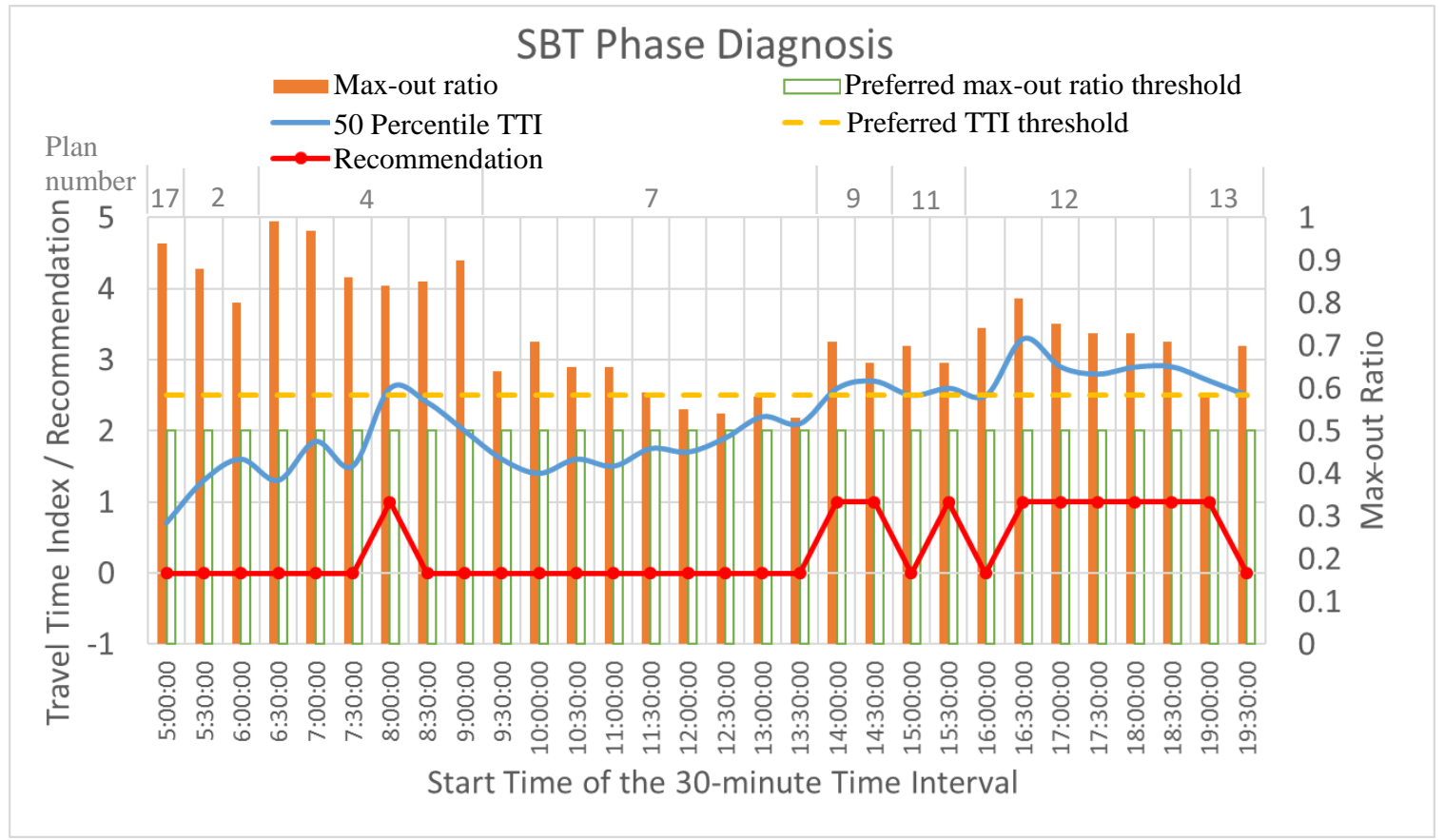

Figure 4-5 Uncoordinated Signal Phase Diagnosis for SBT Movement 


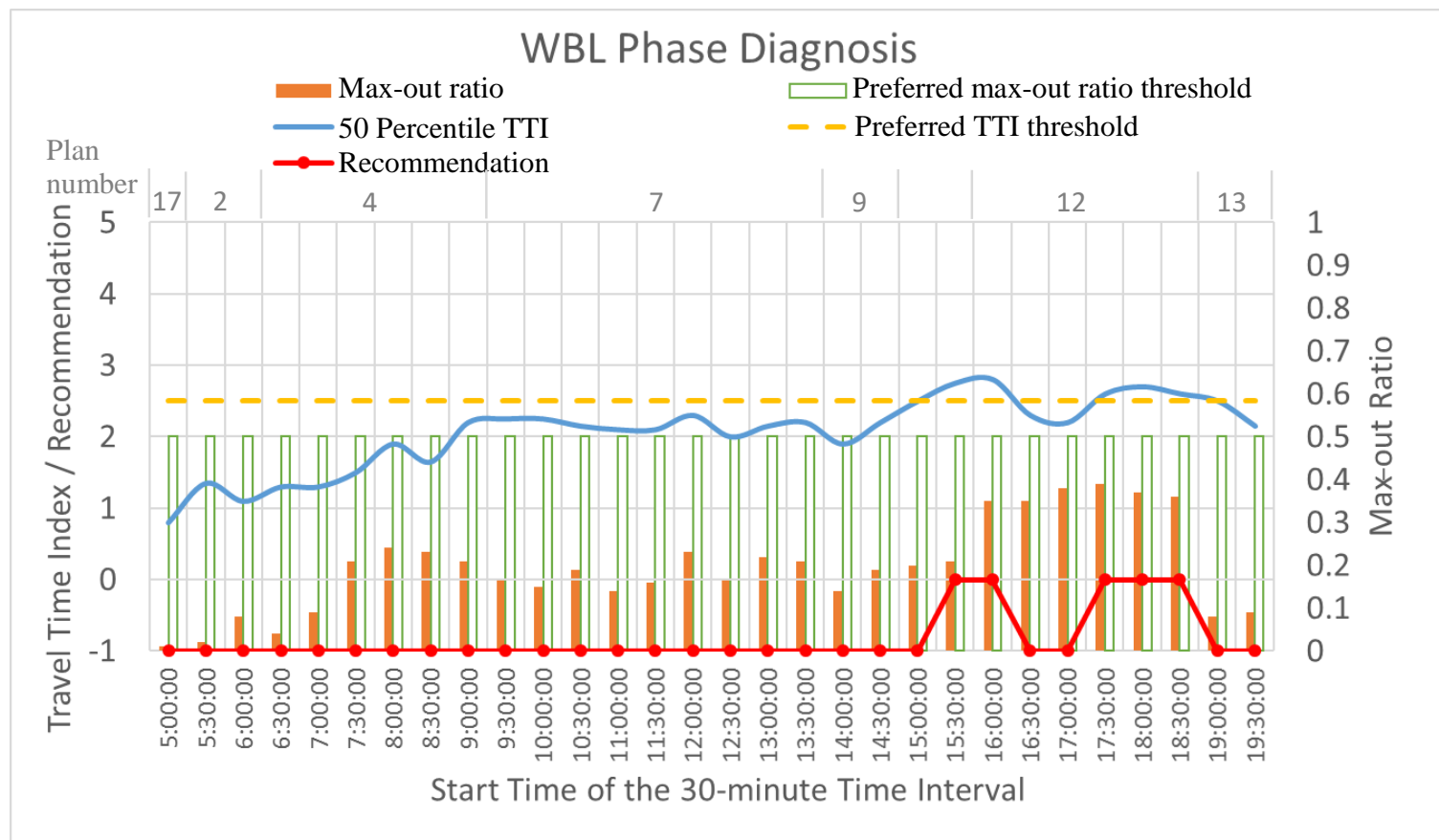

Figure 4-6 Uncoordinated Signal Phase Diagnosis for WBL Movement

\subsubsection{Diagnosis of Coordinated Phases}

As explained in Section 3.5.2, the proposed framework diagnoses the coordinated signal phases based on the TTI, but not on the $M R$. The diagnosis results for the two main street thru movements, EBT and WBT, and their corresponding signal phases (i.e., Phase 2 and Phase 6), are shown in Figure 4-7 and Figure 4-8, respectively. The diagnosis indicates that with the exception of a short time period between 3:30 PM to 3:59 PM, the TTI of the EBT movement is always below the threshold of 2.5. On the other hand, the TTI of the WBT movement mostly reaches or exceeds the threshold between 3:00 PM and 6:59 PM, as confirmed by the results in Figure 3-22. In particular, the TTI of the WBT movement surpasses the threshold between 3:00 PM and 3:59 PM, which is the peak shoulder period. According to Figure 4-8, the diagnosis recommends increasing the corresponding green time for the WBT movement during that time period. This possibly indicates the need to start the peak period plan at 3:00 PM instead 4:00 PM. 


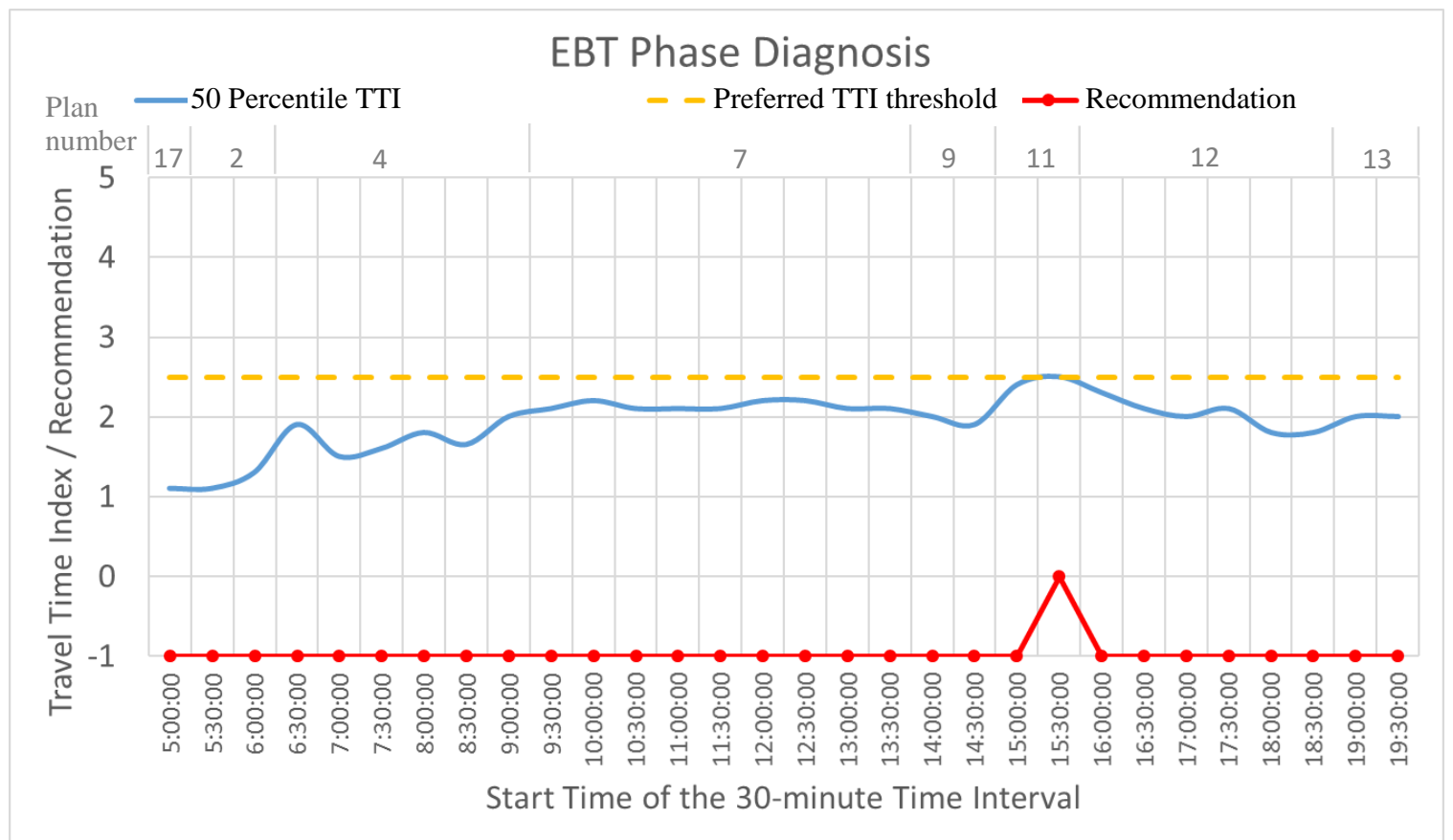

Figure 4-7 Coordinated Signal Phase Diagnosis for EBT Movement

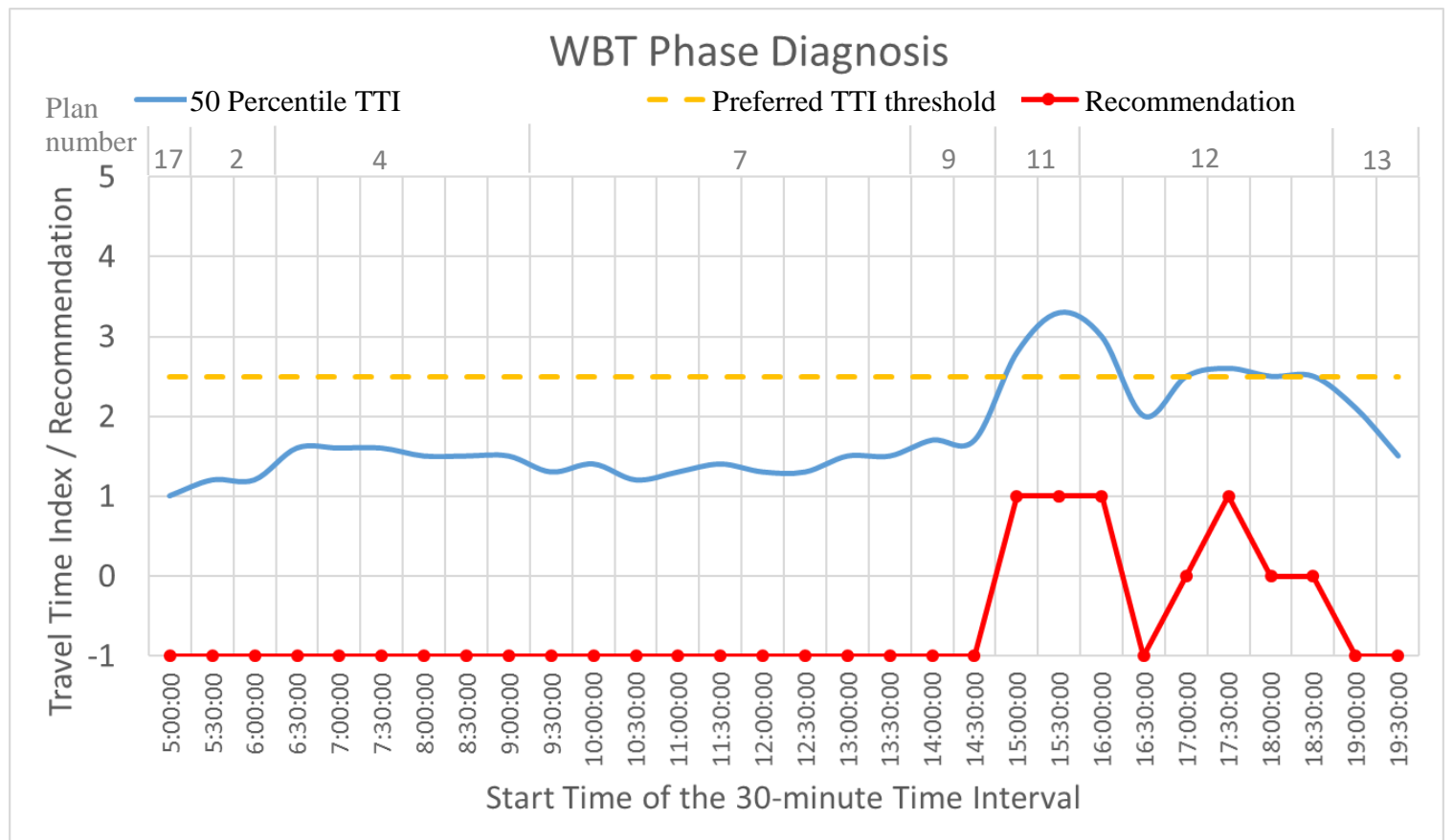

Figure 4-8 Coordinated Signal Phase Diagnosis for WBT Movement

\subsubsection{Diagnosis of Intersection Cycle Length}

Based on the figures of individual phase diagnoses, it can be expected that the cycle length is acceptable in the morning, but it needs to be increased in the PM peak period. After 
examining all individual phases in various time periods, the diagnosis investigates the whole intersection to determine this need, and the results are presented in Figure 4-9. As with the diagnosis results of individual phases, the recommendations of the intersection cycle length are:

- " 1 " recommends to increase the cycle length

- " 0 " recommends to keep the cycle length settings

- "-1" recommends that the cycle length can be reduced

As expected, the diagnosis recommends not changing or decreasing the cycle length most of the day. However, it should be recognized that this recommendation is based on local conditions at the studied intersection. The cycle length is normally selected based on the entire coordinated system, and reducing the cycle length may not be possible. The diagnosis recommends increasing the cycle length between 3:00 PM and 5:59 PM. Since the cycle length included in the Figure 4-9 is already 190 seconds in the PM peak plan between 4:00 PM and 6:59 PM, it is not realistic to introduce a longer cycle length. Longer cycle lengths cause longer queues, and thus are not normally recommended. This may indicate the need for geometry improvement at the studied intersection. On the other hand, between 3:00 PM and 3:59 PM, the utilized cycle length is 170 seconds. In general, the diagnosis implies that the signal plan for the PM peak (i.e., from 4:00 PM to 6:59 PM) should be applied earlier to the so-called peak shoulder period (i.e., from 3:00 PM to 3:59 PM). Perhaps an early start of the PM peak signal plan could prevent traffic breakdown, and the cycle length and geometry would be adequate for the afternoon peak period. 


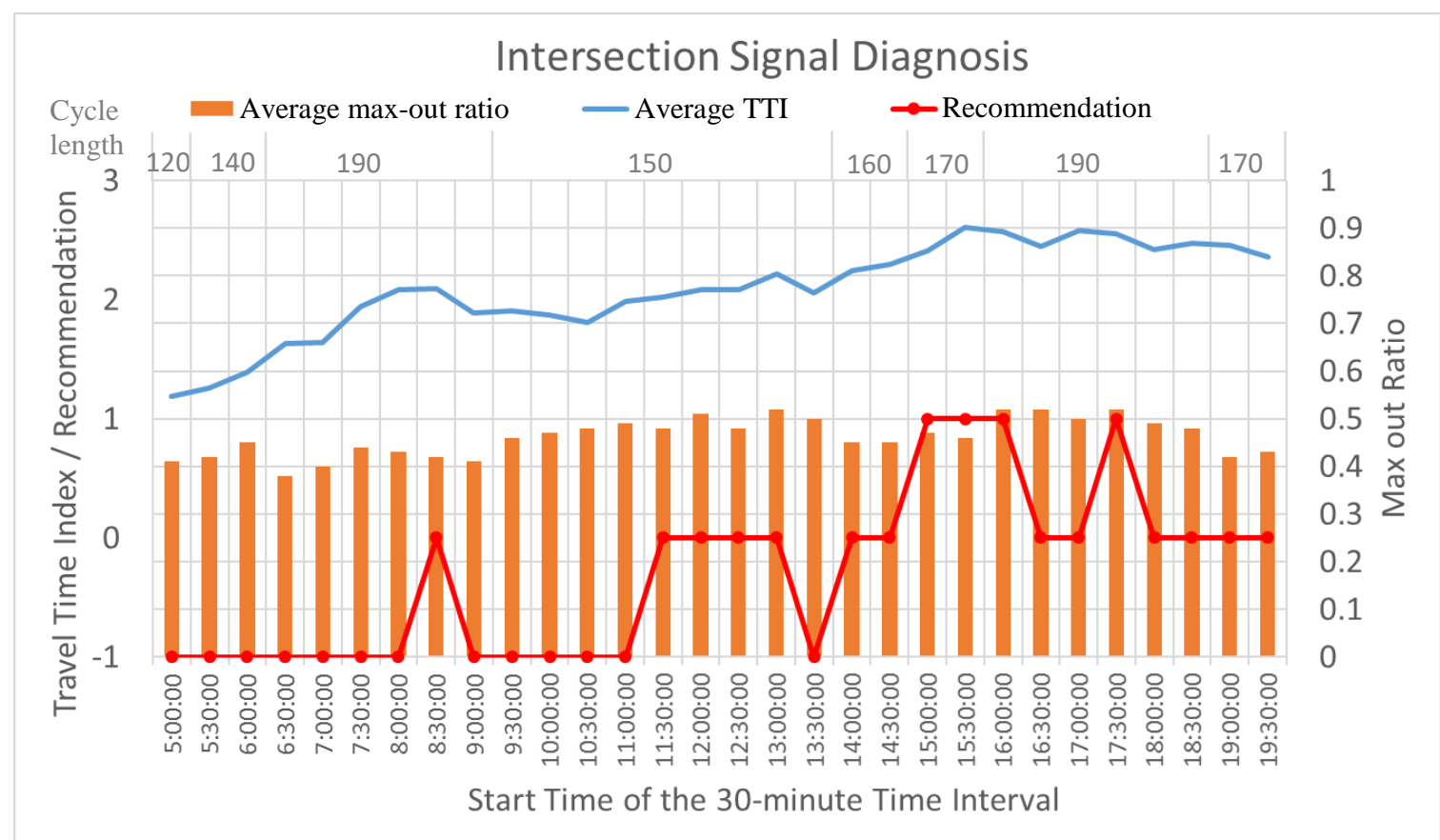

Figure 4-9 Intersection Level Diagnosis

To increase the understanding of the analysis results, the signal operation diagnosis can be presented and visualized another way. An example of the results for the time period between 3:00 PM and 3:59 PM is shown in Figure 4-10. The intersection level diagnosis results are "1" for the hour according to previous discussion, indicating that the cycle length of the corresponding signal plan needs to be increased. The hour between 3:00 PM and 3:59 PM is divided into two time intervals: the interval between 3:00 PM and 3:29 PM, and the interval between 3:30 PM and 3:59 PM. Part (a) in Figure 4-10 shows the recommendations of the diagnosis for the interval between 3:00 PM and 3:29 PM, which suggests that the green times of the WBT and SBL be increased (assigned "1" in the figure). As discussed earlier, the phase for the WBT (Phase 2) movement can borrow green time from the phase for the EBL (Phase 1), which is marked as a candidate for green time decrease (assigned "-1" in the figure). Thus, the diagnosis of Section 1 between 3:00 PM 
and 3:29 PM shown in part (b) in Figure 4-10 is "0," confirming the possible exchange of green times between the two phases in Section 1.

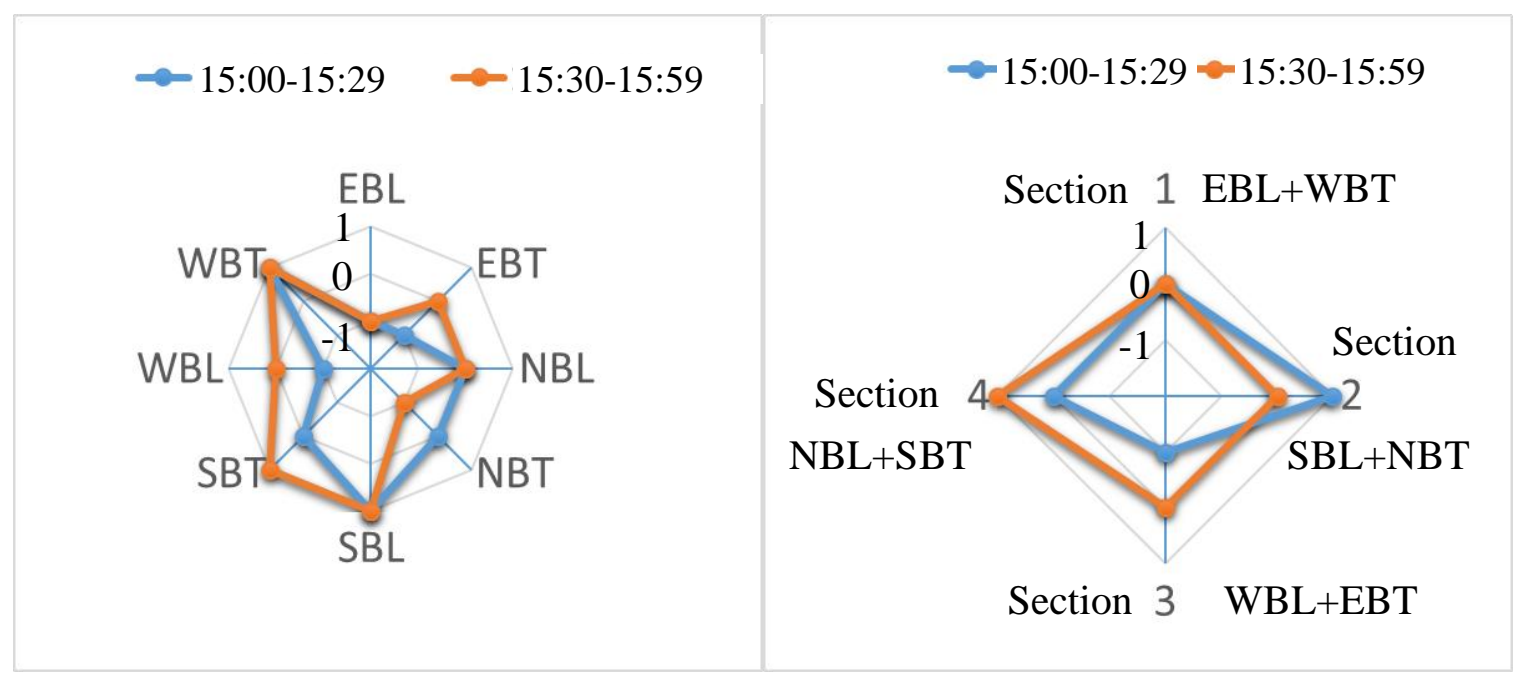

(a)

(b)

Figure 4-10 Individual Phase and Sectional Level Diagnosis Results between 3:00 PM and 3:59 PM

The phase serving the SBL (Phase 2) cannot borrow green time from the phase that serves the NBT (Phase 4), of which there is no surplus green time available. As shown in part (b) of Figure 4-10, Section 2 is flagged because it needs additional green time, as the sectional diagnosis result equals "1." Similar relationships between other individual phase diagnoses (i.e., part (a)) and sectional level diagnoses (i.e., part (b)) in the figure can be derived. Given the fact that Section 2 needs an increase in the sectional green time and other sections need to keep their portions, it is recommended that the cycle length for the time interval between 3:00 PM and 3:29 PM is increased. In summary, the signal operation diagnosis for the time interval between 3:00 PM and 3:29 PM recommends:

- increasing the cycle length for the intersection

- switching green time from Phase 1 (EBL) to Phase 2 (WBT) 
- allocating more green time to Phase 3 (SBL), which can be taken from the increased cycle length

Similarly, the signal operation diagnosis for the time interval between 3:30 PM to 3:59 PM recommends:

- increasing the cycle length for the intersection

- switching green time from Phase 1 (EBL) to Phase 2 (WBT)

- switching green time from Phase 4 (NBT) to Phase 3 (SBL)

- allocating more green time to Phase 8 (SBT), which can be taken from the increased cycle length

\subsection{Comparison of Diagnoses and Field Observations}

A field observation was conducted by the Florida Department of Transportation (FDOT) consultant. The observation report describes the traffic conditions for different movements. The observation periods are divided by time of day, as follows:

- morning peak period from 6:30 AM to 9:29 AM

- midday peak period from 9:30 AM to 1:59 PM

- afternoon peak period from 4:00 PM to 6:59 PM

When compared with the studied time period in this investigation, no information is provided during:

- the pre-morning period between 5:30 AM and 6:29 AM

- the post-midday peak period between 2:00 PM and 3:59 PM that includes the "peak shoulder" period

- the post-afternoon period between 7:00 PM and 7:59 PM. 
The observation report is descriptive, and a partial summary of the description is shown in Table 4-1 and Table 4-2. Table 4-1 categorizes the queue conditions of each movement during different times of day into three levels, as follows: 1) clear, 2) moderate, and 3) heavy. Table 4-2 highlights the movement downstream spillback blockages and adjacent turn-bay spillover blockages, as well as the reasons for the blockage.

Table 4-1 Summary of Queue Levels from Field Observation

\begin{tabular}{|c|c|c|c|}
\hline \multicolumn{4}{|c|}{ Queue Level } \\
\hline Movement / Period & Morning & Midday & Afternoon \\
\hline EBL & Moderate & Clear & Clear \\
\hline EBT & Heavy & Moderate & Moderate \\
\hline WBL & Clear & Moderate & Moderate \\
\hline WBT & Moderate & Clear & Heavy \\
\hline NBL & Heavy & Moderate & Heavy \\
\hline NBT & Moderate & Moderate & Moderate \\
\hline SBL & Heavy & Heavy & Heavy \\
\hline SBT & Clear & Heavy & Heavy \\
\hline
\end{tabular}

The following results from the developed framework of signal operation diagnosis can be confirmed based on the field observations:

- The WBT movement has inferior performance in the PM peak period and requires additional green time.

- Inferior performance of the SBL and SBT movements begins at midday, and additional green time or capacity improvements are needed for these movements. At midday, the SBL and SBT movements should receive additional green time, which can be taken from the EBL and WBT.

- In the PM peak period, there is an opportunity to take some green time from the EBL movement and add it to the WBT movement. 
- The NBT movement experiences travel time increase at the studied intersection between 8:00 AM and 8:59 AM because of the downstream intersection of Southwest 4th Street and Southwest 107th Avenue, which is located within a school zone.

Table 4-2 Summary of Movement Blockages from Field Observation

\begin{tabular}{|c|c|c|c|}
\hline \multicolumn{4}{|c|}{ Movement Blocked by } \\
\hline Movement / Period & Morning & Midday & Afternoon \\
\hline EBL & EBT/Downstream & & \\
\hline EBT & & & \\
\hline WBL & & & \\
\hline WBT & & & Downstream \\
\hline NBL & Downstream & & NBL \\
\hline NBT & & & \\
\hline SBL & & & \\
\hline SBT & & & \\
\hline
\end{tabular}

On the other hand, some diagnoses cannot be confirmed by the observations. The unconfirmed diagnosis results are listed below:

- The diagnoses between 2:00 PM and 4:00 PM cannot be confirmed due to lack of field observations.

- In the morning, heavy queues are reported for the SBL movement, but the diagnosis does not indicate the need for green time increase.

- Heavy queuing of NBL is observed in the morning and afternoon, and moderate queuing is observed at midday. However, the diagnosis identified inferior signal operation performance of the NBL movement only during the midday/lunch peak period. 
- In the morning, cycle failures are observed for the NBL, but the max-out ratios calculated in this study are relatively low, and thus no green time increase is recommended.

\subsection{Summary}

The developed framework of signal operation diagnosis was implemented in this chapter at an intersection with a coordinated dual-ring eight-phase signal control. The diagnostic scheme was written in Visual Basic ${ }^{\circledR}$ language and Structured Query Language (SQL). The $50^{\text {th }}$ percentile $T T I$ was selected for the diagnosis. The preferred $T T I$ threshold was set to 2.5 , and the $M R$ threshold was set to 0.5 . The diagnosis inspected the uncoordinated phases and coordinated phases separately, then combined the results for all individual phases to diagnose the cycle length and capacity needs of the intersection. The diagnosis results are summarized in Table 4-3.

In the morning between 8:00 AM to 9:00 AM, the green time of the phases for NBT and SBT movement is recommended to increase due to the school zone near the intersection of Southwest 4th Street and Southwest 107th Avenue. Green time increase is recommended for the SBL movement from 11:30 AM. The SBT movement is recommended to increase the corresponding phase green time from 2:00 PM. The NBL movement is recommended to increase its phase green time at midday between 12:00 PM and 1:30 PM. The phase green time for the WBT movement is recommended to increase in the afternoon between 3:00 PM to 4:30 PM and between 5:30 PM and 6:00 PM. The cycle length of the entire intersection is recommended to increase between 3:00 PM and 4:30 PM and between 5:30 PM and 6:00 PM. 
Table 4-3: Summary of Signal Operation Diagnosis Results

\begin{tabular}{|c|c|c|c|c|c|c|c|c|c|}
\hline $\begin{array}{l}\text { Strat Time } \\
\text { of Time }\end{array}$ & \multicolumn{2}{|c|}{ Section 1} & \multicolumn{2}{|c|}{ Section 2} & \multicolumn{2}{|c|}{ Section 3} & \multicolumn{2}{|c|}{ Section 4} & \multirow{3}{*}{$\begin{array}{c}\text { Intersection } \\
\begin{array}{c}\text { Cycle } \\
\text { Length }\end{array}\end{array}$} \\
\hline Phase & $\begin{array}{c}\text { Phase } \\
1\end{array}$ & $\begin{array}{c}\text { Phase } \\
2\end{array}$ & $\begin{array}{c}\text { Phase } \\
3\end{array}$ & $\begin{array}{c}\text { Phase } \\
4\end{array}$ & $\begin{array}{c}\text { Phase } \\
5\end{array}$ & $\begin{array}{c}\text { Phase } \\
6\end{array}$ & $\begin{array}{c}\text { Phase } \\
7\end{array}$ & $\begin{array}{c}\text { Phase } \\
8\end{array}$ & \\
\hline Movement & EBL & WBT & SBL & NBT & WBL & EBT & NBL & SBT & \\
\hline $5: 00: 00$ & -1 & -1 & -1 & 0 & -1 & -1 & -1 & 0 & -1 \\
\hline $5: 30: 00$ & -1 & -1 & -1 & 0 & -1 & -1 & -1 & 0 & -1 \\
\hline $6: 00: 00$ & -1 & -1 & 0 & 0 & -1 & -1 & -1 & 0 & -1 \\
\hline $6: 30: 00$ & -1 & -1 & 0 & 0 & -1 & -1 & -1 & 0 & -1 \\
\hline $7: 00: 00$ & -1 & -1 & 0 & 0 & -1 & -1 & -1 & 0 & -1 \\
\hline $7: 30: 00$ & -1 & -1 & 0 & 0 & -1 & -1 & -1 & 0 & -1 \\
\hline $8: 00: 00$ & -1 & -1 & 0 & 0 & -1 & -1 & -1 & 1 & -1 \\
\hline $8: 30: 00$ & -1 & -1 & 0 & 1 & -1 & -1 & -1 & 0 & 0 \\
\hline 9:00:00 & -1 & -1 & 0 & 0 & -1 & -1 & -1 & 0 & -1 \\
\hline 9:30:00 & -1 & -1 & 0 & 0 & -1 & -1 & -1 & 0 & -1 \\
\hline 10:00:00 & -1 & -1 & -1 & 0 & -1 & -1 & -1 & 0 & -1 \\
\hline $10: 30: 00$ & -1 & -1 & -1 & 0 & -1 & -1 & -1 & 0 & -1 \\
\hline 11:00:00 & -1 & -1 & -1 & 0 & -1 & -1 & 0 & 0 & -1 \\
\hline $11: 30: 00$ & -1 & -1 & 1 & 0 & -1 & -1 & 0 & 0 & 0 \\
\hline $12: 00: 00$ & -1 & -1 & 1 & 0 & -1 & -1 & 1 & 0 & 0 \\
\hline $12: 30: 00$ & -1 & -1 & 1 & 0 & -1 & -1 & 1 & 0 & 0 \\
\hline 13:00:00 & -1 & -1 & 1 & 0 & -1 & -1 & 1 & 0 & 0 \\
\hline 13:30:00 & -1 & -1 & 0 & 0 & -1 & -1 & 0 & 0 & -1 \\
\hline 14:00:00 & -1 & -1 & 1 & 0 & -1 & -1 & 0 & 1 & 0 \\
\hline $14: 30: 00$ & -1 & -1 & 1 & 0 & -1 & -1 & -1 & 1 & 0 \\
\hline 15:00:00 & -1 & 1 & 1 & 0 & -1 & -1 & 0 & 0 & 1 \\
\hline $15: 30: 00$ & -1 & 1 & 1 & -1 & 0 & 0 & 0 & 1 & 1 \\
\hline 16:00:00 & -1 & 1 & 1 & 0 & 0 & -1 & -1 & 0 & 1 \\
\hline $16: 30: 00$ & -1 & -1 & 1 & 0 & -1 & -1 & -1 & 1 & 0 \\
\hline 17:00:00 & -1 & 0 & 1 & 0 & -1 & -1 & 0 & 1 & 0 \\
\hline $17: 30: 00$ & -1 & 1 & 1 & 1 & 0 & -1 & -1 & 1 & 1 \\
\hline 18:00:00 & -1 & 0 & 1 & 0 & 0 & -1 & -1 & 1 & 0 \\
\hline 18:30:00 & -1 & 0 & 1 & 0 & 0 & -1 & -1 & 1 & 0 \\
\hline 19:00:00 & -1 & -1 & 1 & 1 & -1 & -1 & -1 & 1 & 0 \\
\hline $19: 30: 00$ & -1 & -1 & 1 & 0 & -1 & -1 & -1 & 0 & 0 \\
\hline
\end{tabular}


The diagnosis results are compared to the field observations and the comparison indicated that the system is capable of identifying improvement directions to support decision-makers in their retiming efforts. The system is able to provide information on the current signal operation by answering the following questions: 1) Is there a need to change the signal plan? 2) How can the signal plan be changed?

It should be noted that the same thresholds of the utilized measures (TTI and $M R$ ) were used for all movements in this implementation. Nevertheless, different thresholds can be selected by decision-makers based on their experience and the local situations and priorities at the intersection where the diagnosis is performed. The impacts of selecting different thresholds will be discussed in the next chapter. 


\section{CHAPTER 5}

\section{THRESHOLDS SELECTION}

\subsection{Introduction}

In the previous chapter, the proposed framework of signal operation diagnosis was implemented in the studied intersection with the same TTI threshold and $M R$ threshold for all turning movements. The appropriateness of introducing the same thresholds for different movements is arguable, since the current practice intends to provide better service to the main street traffic streams, whose signal phases are often coordinated. Whether to apply different thresholds to various turning movements or not and the selection of the appropriate thresholds are decisions that the users can make. The previous chapter provides a starting point on implementing the framework. This chapter continues such efforts and attempts to select different thresholds for various signal phases.

\subsection{Comparison of Thresholds}

The thresholds of the developed framework include 1) TTI thresholds; and 2) $M R$ thresholds. Choosing different thresholds for various signal phases works similar to applying different weights to the turning movements. For example, lower thresholds are selected for the main street instead of the thresholds preferred for the minor street. Similarly, the coordinated phases can be assigned lower thresholds, and the uncoordinated phases are given thresholds with higher values. Additionally, other than applying different thresholds to different movements, the thresholds can vary by time of day.

Selecting different thresholds can lead to different diagnosis results. In this chapter, the framework of signal operation diagnosis is implemented in the same studied 
intersection of Southwest 8th Street at Southwest 107th Avenue in Miami, Florida, with a different set of thresholds, in comparison to the previous implementation discussed in Chapter 4. The thresholds applied in both implementations are summarized in Table 5-1.

Table 5-1 Thresholds Utilized in Different Implementations

\begin{tabular}{|c|c|c|c|c|c|}
\hline Phase & Movement & $\begin{array}{c}\text { TTI Threshold } \\
\text { Set 1 }\end{array}$ & $\begin{array}{c}\text { TTI Threshold } \\
\text { Set 2 }\end{array}$ & $\begin{array}{c}\text { MR Threshold } \\
\text { Set 1 }\end{array}$ & $\begin{array}{c}\text { MR Threshold } \\
\text { Set 2 }\end{array}$ \\
\hline 1 & EBL & 2.5 & 3.0 & 0.5 & 0.7 \\
\hline 2 & WBT & 2.5 & 2.0 & N/A & N/A \\
\hline 3 & SBL & 2.5 & 3.5 & 0.5 & 0.7 \\
\hline 4 & NBT & 2.5 & 2.5 & 0.5 & 0.7 \\
\hline 5 & WBL & 2.5 & 3.0 & 0.5 & 0.7 \\
\hline 6 & EBT & 2.5 & 2.0 & N/A & N/A \\
\hline 7 & NBL & 2.5 & 3.5 & 0.5 & 0.7 \\
\hline 8 & SBT & 2.5 & 2.5 & 0.5 & 0.7 \\
\hline
\end{tabular}

The TTI threshold set 2 tested in this chapter utilized different values for various movements. The new thresholds favor movements in the east-west direction (main street), compared to the movements along the north-south direction. They also favor the movements compared to left turns. Additionally, the preferred $M R$ threshold of 0.5 is replaced by 0.7 , indicating a stricter rule to identify the need for green time increase.

\subsection{Comparison of the Diagnosis Results}

The diagnosis results for each individual signal phase with the newly selected thresholds are compared with the previous results and presented in Figure 5-1 through Figure 5-9. With the newly selected thresholds, higher TTI thresholds are set for the left-turn movements, which make it more difficult to recommend green time increase for those movements. Moreover, a higher $M R$ threshold has a smaller chance of requesting extra green time, since the probability of violating the new $M R$ threshold is lower. Consequently, 
there should be more recommendations to either keep the current green times or be flagged as candidates for green time decrease.

The above expectations are confirmed in Figure 5-1, Figure 5-2, Figure 5-4, and Figure 5-6, as the blue lines tend to stay away from the recommendation of "1" (i.e., increasing green time) and vary between " 0 " (i.e., keeping green time) and "-1" (i.e., being flagged as a candidate for green time decrease). More specifically, as shown in Figure 5-1, the diagnosis flags the phase for the EBL movement (Phase 1) as a candidate for decreasing its green time during the entire studied period.

The diagnosis results for the thru movements in the north-south direction are presented in Figure 5-3 and Figure 5-5. Although the newly selected TTI thresholds are exactly the same as the previous ones, the frequency of the recommendation to increase the corresponding green time drops due to the higher $M R$ threshold. On the contrary, the frequency of recommending green time increase rises for the thru movements in the eastwest direction, as shown in Figure 5-7 and Figure 5-8, due to the lower TTI threshold.

The findings of the intersection level diagnosis are a combination of the diagnoses of all individual signal phases at the studied intersection, as shown in Figure 5-9. The diagnosis results of the two scenarios with different thresholds are clearly different. Upon applying the newly selected thresholds, the diagnosis does not recommend cycle length increase for the intersection, due to the low frequency that the individual phases are flagged for green time increase. 


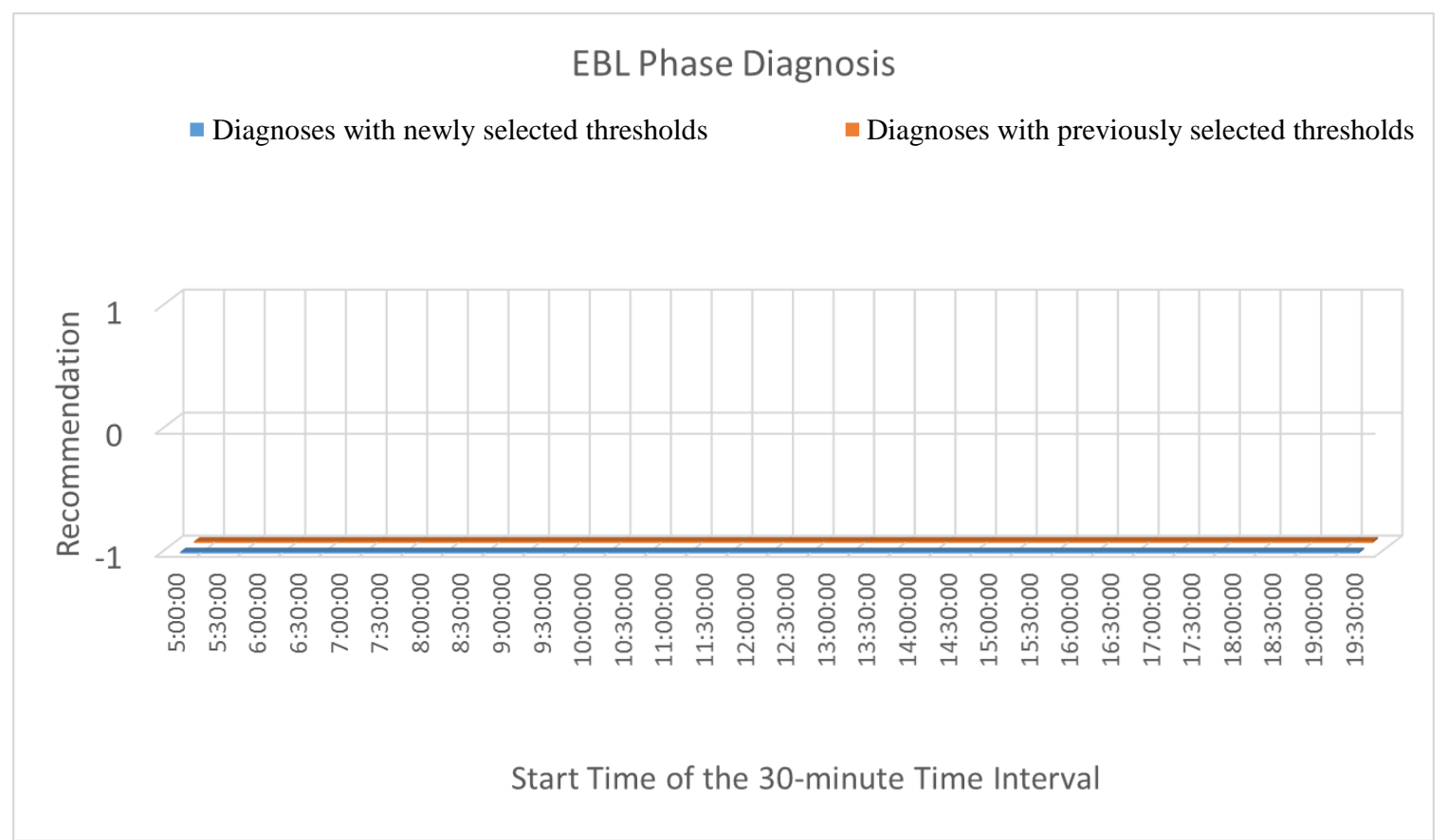

Figure 5-1 Comparison of Signal Phase Diagnosis Results for EBL Movement

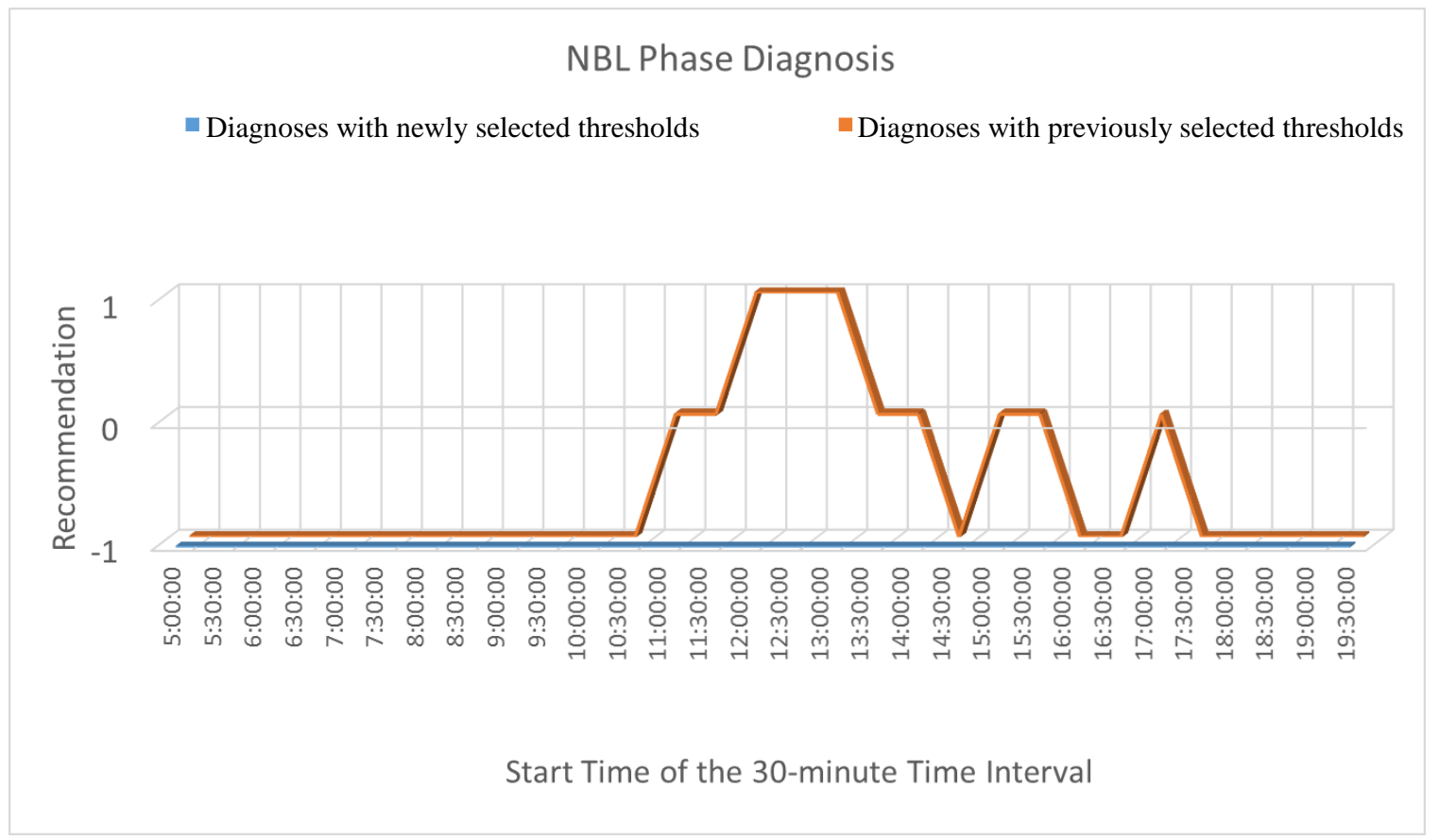

Figure 5-2 Comparison of Signal Phase Diagnosis Results for NBL Movement 


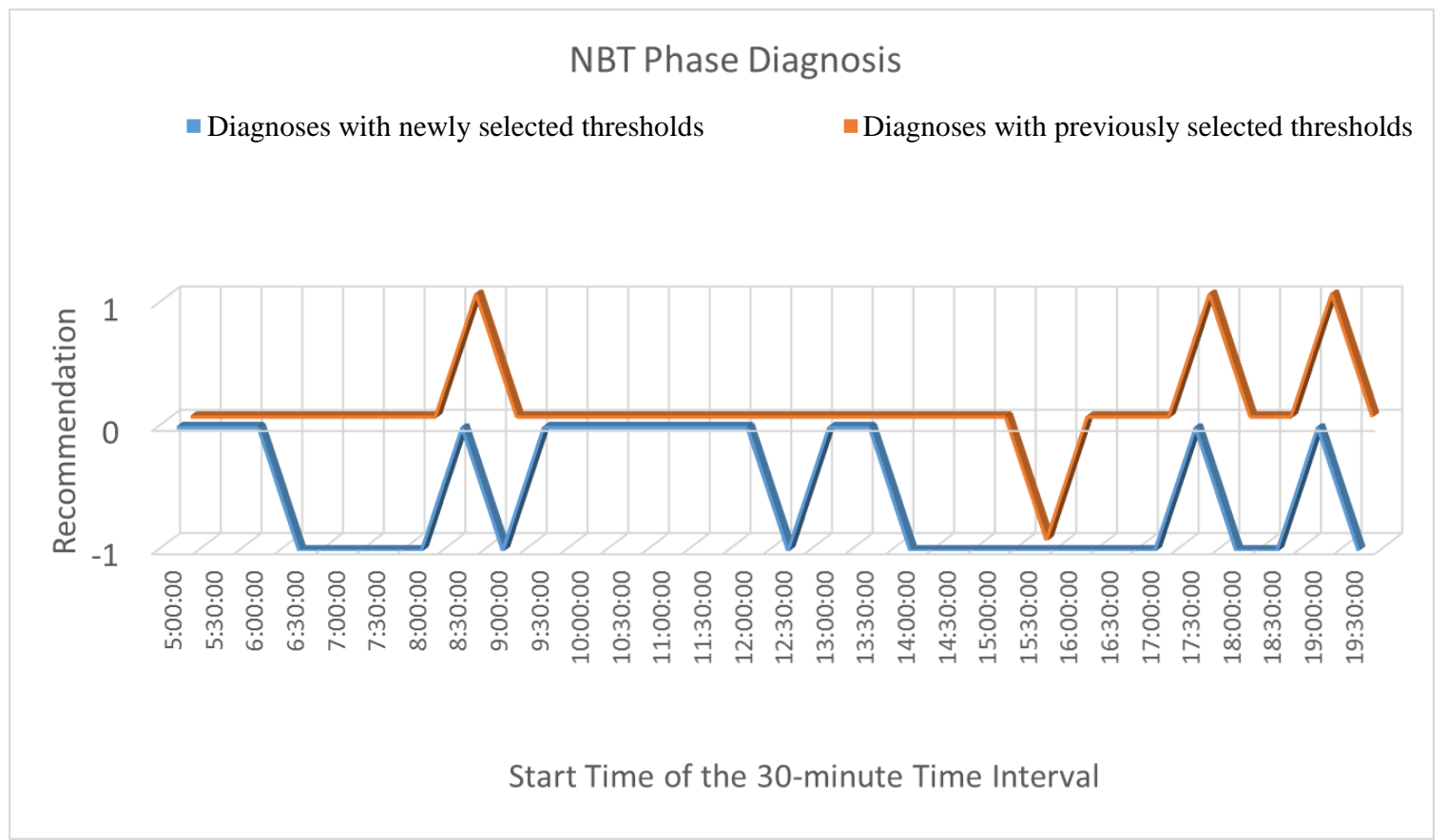

Figure 5-3 Comparison of Signal Phase Diagnosis Results for NBT Movement

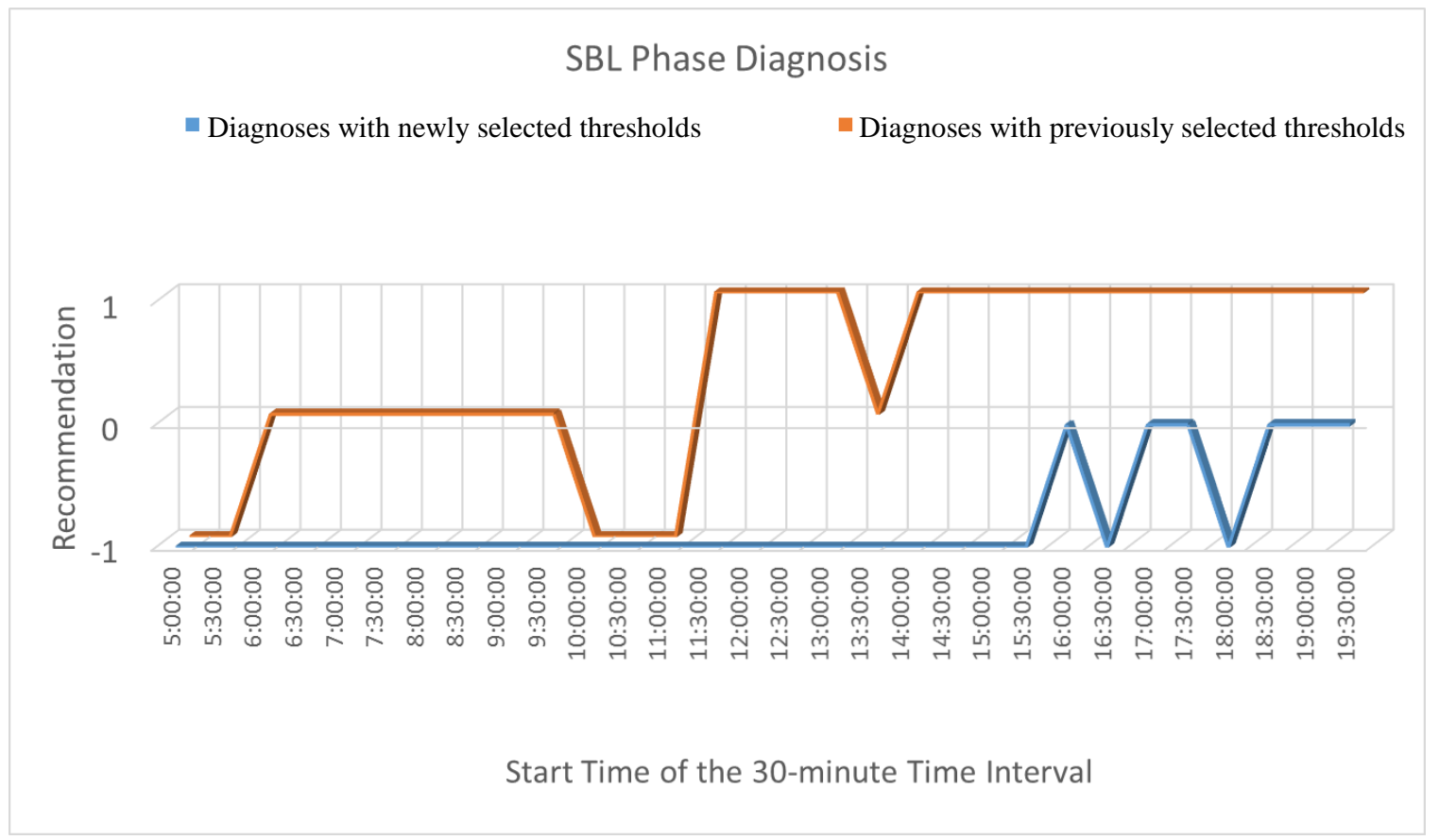

Figure 5-4 Comparison of Signal Phase Diagnosis Results for SBL Movement 


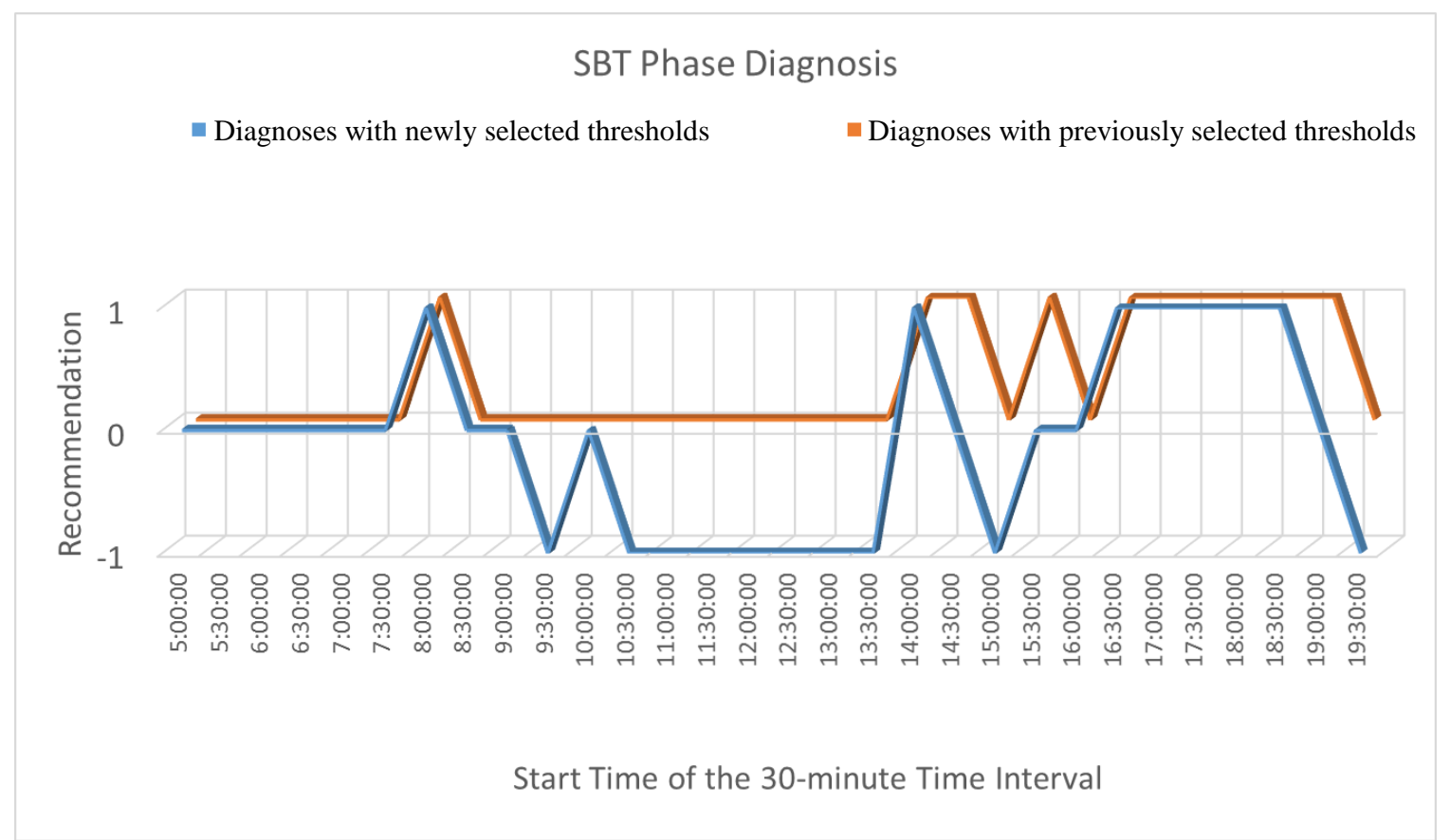

Figure 5-5 Comparison of Signal Phase Diagnosis Results for SBT Movement

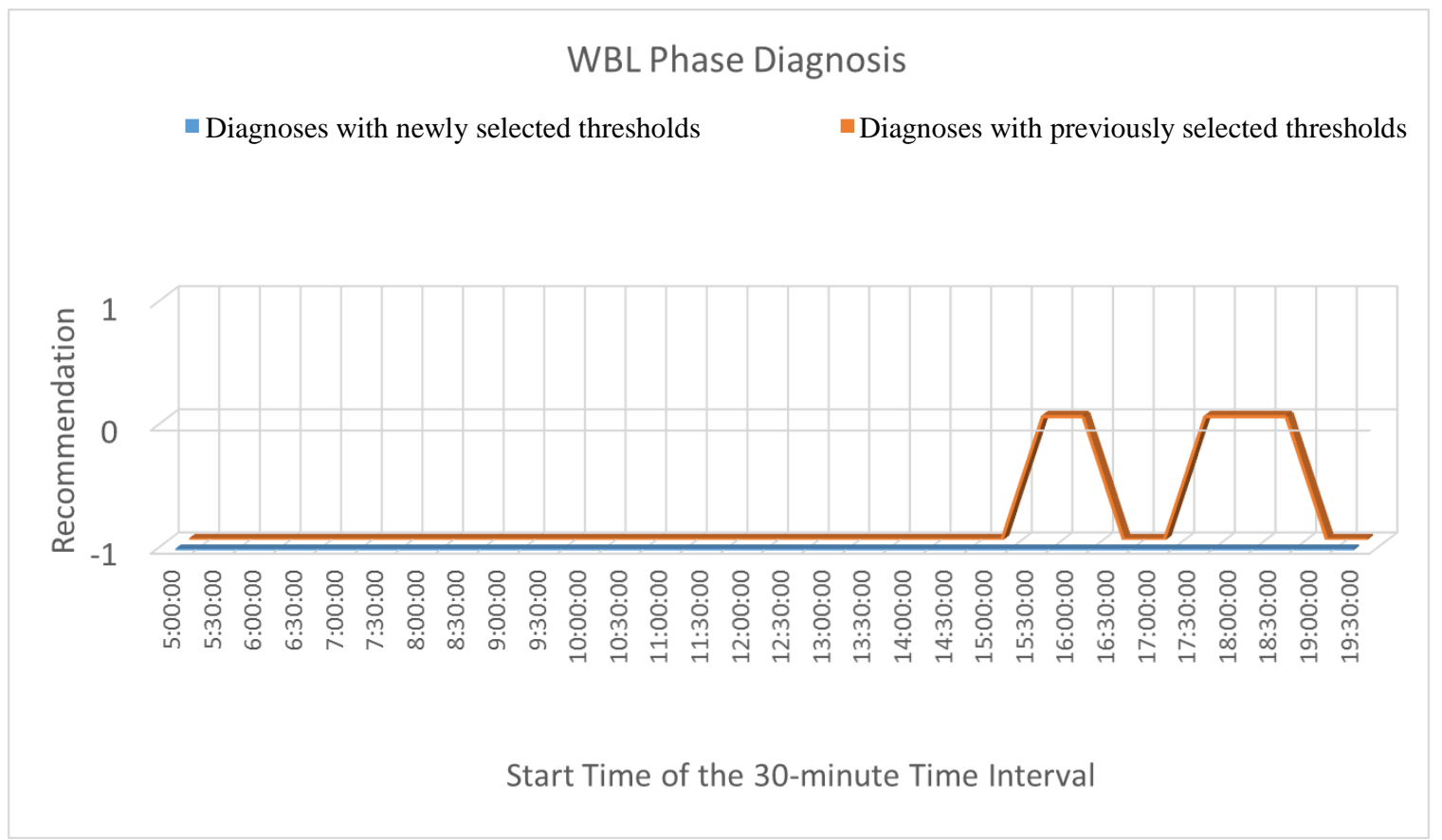

Figure 5-6 Comparison of Signal Phase Diagnosis Results for WBL Movement 


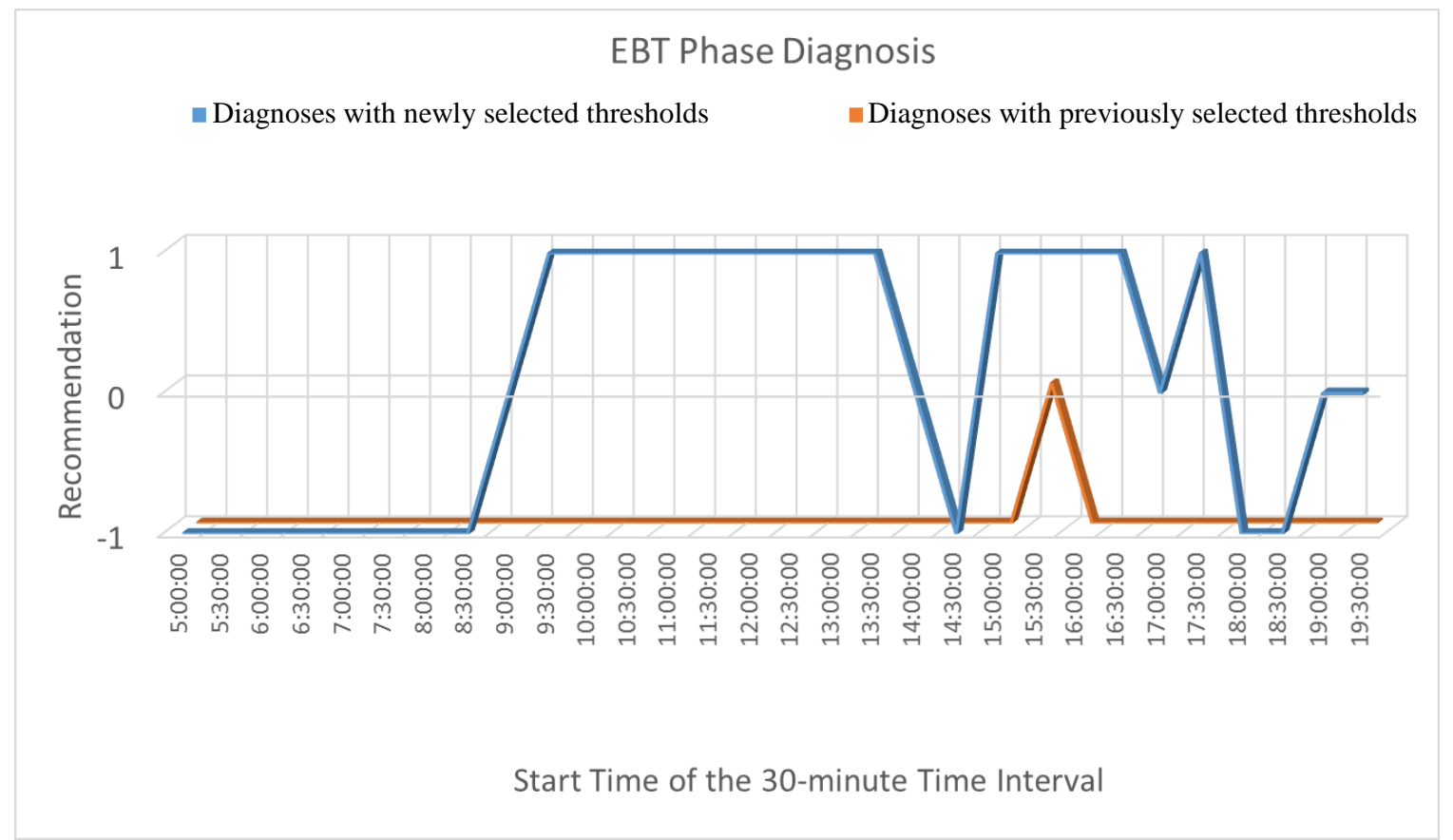

Figure 5-7 Comparison of Signal Phase Diagnosis Results for EBT Movement

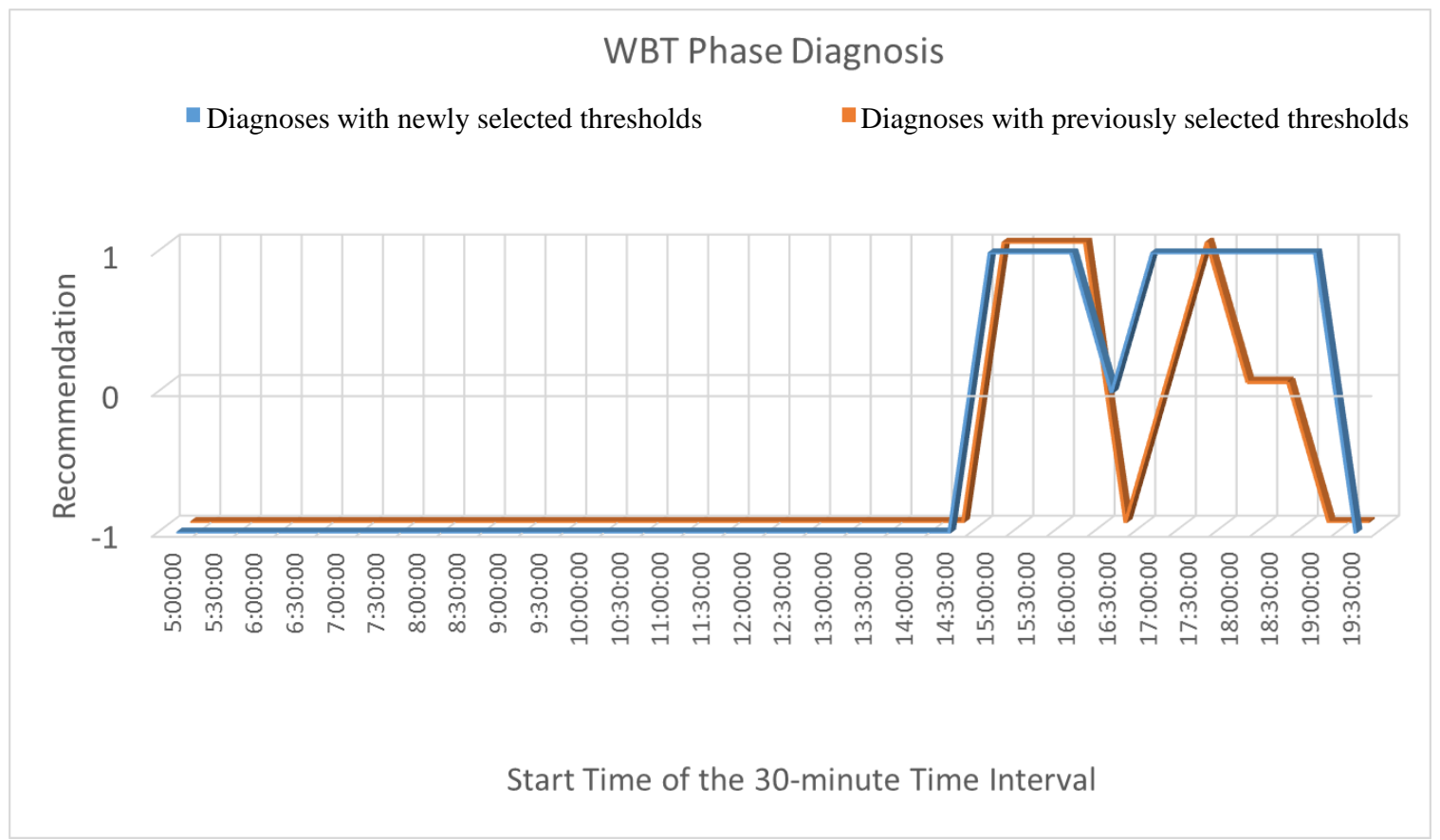

Figure 5-8 Comparison of Signal Phase Diagnosis Results for WBT Movement 


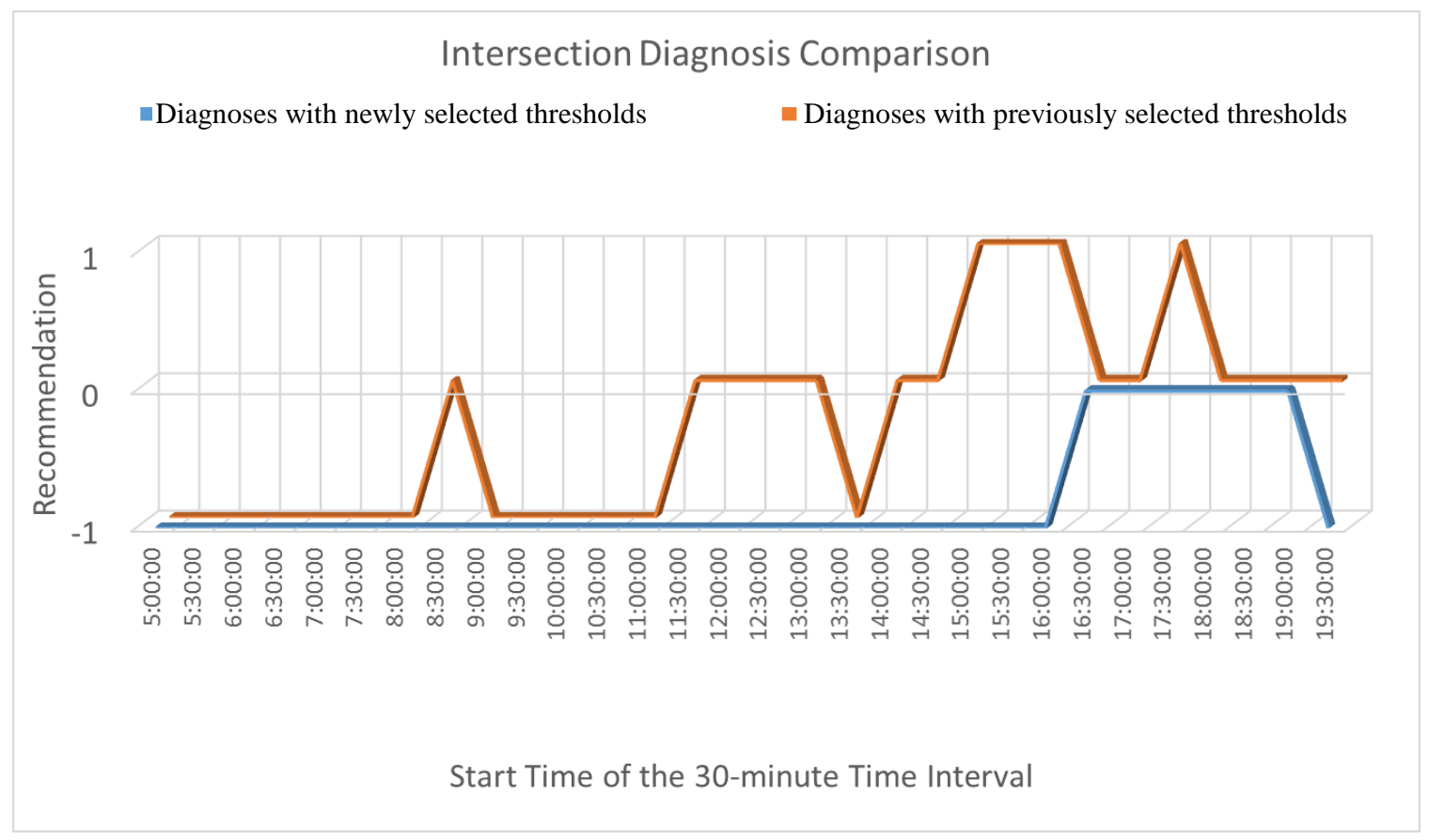

\section{Figure 5-9 Comparison of Signal Diagnosis Results for the Intersection}

\subsection{Summary}

The proposed framework of signal operation diagnosis utilizes two thresholds, the TTI threshold and $M R$ threshold, for various movements and time periods. The previous chapter provided a starting point on implementing the framework of signal operation diagnosis in a real-world intersection with the application of equivalent $T T I$ thresholds and $M R$ thresholds for all turning movements. This chapter presents an example of selecting different thresholds in the signal diagnosis. Different $T T I$ thresholds were selected for various movements, with lower thresholds for the main traffic stream, as well as the thru movements. In addition, a higher $M R$ threshold is applied to all phases. The diagnosis results showed less frequent requests for green time increase and more for green time decrease for the uncoordinated phases. The intersection level diagnoses were also dramatically different than those presented in the previous chapter, with no cycle length increase, per the recommendation of the diagnosis. 


\section{CHAPTER 6}

\section{SUMMARY, CONCLUSIONS, AND RECOMMENDATIONS}

\subsection{Summary and Conclusions}

The current practices of signal retiming are periodic and based on limited and aggregated turning movement counts. There is a need for continuous signal operation monitoring to ensure effective and efficient control of signalized arterials. This dissertation developed a framework of automatic signal operation diagnosis to support the decision-making process by identifying problems in existing signal controls, evaluating the needs of signal retiming, and providing directions for the retiming process. The developed framework utilized data from relatively low-cost Automatic Vehicle Identification (AVI) detection technology such as Wi-Fi and Bluetooth sensors, in combination with data from current signal controllers to provide information for diagnosing signal operations, without using high-resolution event data.

Identification of vehicles that traverse the intersection without intermediate stops for other purposes under recurrent traffic conditions is critical to the developed the framework. With the appropriate installation of sensors at various locations and the application of data matching algorithms, trips between different intersections were identified. Travel times were calculated as the difference between the timestamps of different detection stages from a pair of sensors. By applying multiple data filtering algorithms based on the calculated travel times, including introducing the Density-Based Spatial Clustering of Applications with Noise (DBSCAN) algorithm to the data filtering process, it was possible to identify a set of traversals for use in the analysis. This data was 
aggregated and summarized as Travel Time Index (TTI) with different percentiles and was used to generate a series of Cumulative Density Function (CDF) plots for the assessment of traffic conditions at an intersection. Initially, these CDF plots were used for "manual" identification of problems in signal operation.

As discussed above, aside from utilizing the TTI estimated based on AVI detections to assess traffic conditions, Max-out Ratio $(M R)$ extracted from the historical signal timing records was introduced as another variable for use in combination with $T T I$ to reflect the status of the signal operations. The developed framework combined $T T I$ and $M R$ to automatically evaluate whether or not the green time for an individual phase is adequate to serve the corresponding movement. After the completion of diagnosing all individual phases at a given intersection, the framework allowed the assessment of the cycle length and/or capacity adequacy for the entire intersection with the examination of the diagnosis results for all signal phases.

The developed framework was implemented at a studied intersection under a typical dual-ring eight-phase coordinated signal control. The framework diagnosed the signal operation every 30 minutes during the studied time period, with the same preferred TTI threshold and MR threshold for all movements. The diagnosis evaluated each of the eight signal phases and made one of the following recommendations for each phase:

1. increase its green time

2. keep its green time allocation

3. flag the phase as a candidate for green time decrease.

The diagnosis then evaluated the cycle length and recommended either to increase or to keep the current setting. 
A different set of thresholds were applied in another implementation of the framework to diagnose the same signal operation, for the purpose of understanding the impact of the selection of the thresholds. The new set included different TTI thresholds for the movements, giving higher priority to the main street and thru movements, and a higher $M R$ threshold. The results of the analysis showed the expected trend of the diagnosis results caused by varying the set of thresholds.

\subsection{Research Contributions}

Identifying an existing signal control problem is a challenging task in arterial management and operations. The existing efforts on signal monitoring and diagnosis are limited and sporadic. Recently, advanced efforts to address this problem were based on high-resolution controller and detector data, which are still difficult to obtain from most existing intersection control systems. This research developed a framework of automatic signal operation diagnosis utilizing a combination of low-cost AVI data and signal records from prevailing signal controllers. A robust diagnosis scheme was also developed to inspect the green time allocation of individual signal phases, as well as the cycle length of the entire signal plan.

\subsection{Recommendations for Future Research}

Although a completed framework of signal operation diagnosis was developed in this dissertation, the system development will benefit from additional development efforts. Future studies on the following areas will help improve the framework:

- The developed framework was designed to use different AVI data. However, only Wi-Fi data were investigated in this dissertation due to the type of data availability. 
The data filtering methodology may be different among various AVI data. Therefore, future investigations need to be conducted on the appropriate utilization of other AVI data such as Bluetooth readings. The use of third party vendors should also be investigated for this purpose.

- The DBSCAN algorithm was applied to the data filtering process as part of the framework. The two parameters, $\varepsilon$ and minPts, were manually selected based on the actual data obtained from the field. These parameters may not be perfect for all situations and thus need further investigation. The optimization or generalization technique of the estimation of these two parameters will provide a more efficient and precise identification of traversals.

- When applying the DBSCAN algorithm to the data filtering process, only trips in one cluster with the shortest travel times were identified as valid traversals. Future studies are needed on the potential of using data from more than one cluster. It is possible that traversals are contained in multiple clusters, especially when cycle failures occur.

- The developed framework assessed the green time allocation and cycle length of the signal plans. However, it did not take traffic progression and the corresponding offsets into account. Due to the difficulties in synchronizing the Wi-Fi sensors with the signal controller, the arrival types could not be studied in this dissertation. Future work is needed in this area.

- The framework of signal operation diagnosis examined the decision performance measures (i.e., TTI and $M R$ ) by comparing them to thresholds with set values. However, future research should also consider specifying each threshold as a range 
rather than a fixed point. This will prevent making two completely different decisions for very similar measurements. 


\section{REFERENCES}

American Association of State Highway and Transportation Officials (AASHTO). Signal Performance Metrics. Utah Department of Transportation.

http://udottraffic.utah.gov/atspm/. Accessed November 18, 2016.

Balke, K., H. Charara, and R. Parker. Development of A Traffic Signal Performance Measurement System (TSPMS). FHWA/TX-05/0-4422-2. Federal Highway

Administration (FHWA), U.S. Department of Transportation, 2005.

Bullock, D., R. Clayton, J. Mackey, S. Misgen, A. Stevens, J. Sturdevant, and M. Taylor. Automated Traffic Signal Performance Measures: Helping Traffic Engineers Manage Data to Make Better Decisions. ITE Journal, Vol. 84, No. 3, 2014, pp. 33-39.

Chen, C. Freeway Performance Measurement System (PeMS). Dissertation, University of California, Berkeley, CA, 2002.

Cheu, R., D. Lee, and C. Xie. An Arterial Speed Estimation Model Fusing Data from Stationary and Mobile Sensors. Presented at Intelligent Transportation Systems Conference. Oakland, CA, 2001.

Courage, K., R. Showers, J. Harriot, W. Schilling, and K. Godbey. Improved Methods for Measuring Travel Time on Arterial Streets. FL/DOT 99700-3519-119. Florida

Department of Transportation, 1998

Wikipedia contributors. Cumulative Distribution Function. Wikipedia: The Free Encyclopedia. https://en.wikipedia.org/wiki/Cumulative_distribution_function. Accessed September 16, 2016.

Dailey, D., and F. Cathey. AVL-Equipped Vehicles as Traffic Probe Sensors. WA-RD 534.1. Federal Highway Administration (FHWA), U.S. Department of Transportation, 2002.

Day, C., and D. Bullock. Performance Based Management of Arterial Traffic Signal Systems. NCHRP Project 3-79a. National Cooperative Highway Research Program (NCHRP), Transportation Research Board, National Research Council, 2010.

Day, C., R. Haseman, H. Premachandra, T. Brennan, J. Wasson, and J. Sturdevant. Evaluation of Arterial Signal Coordination: Methodologies for Visualizing HighResolution Event Data and Measuring Travel Time. Transportation Research Record: Journal of the Transportation Research Board, No. 2192, Transportation Research Board of the National Academies, Washington, D.C., 2009, pp. 37-49.

Frechette, L., and A. Khan. Bayesian Regression-Based Urban Traffic Models. Transportation Research Record: Journal of the Transportation Research Board, No. 
1644, Transportation Research Board of the National Academies, Washington, D.C., 1998, pp. 157-165.

Koonce, P., L. Rodegerdts, K. Lee, S. Quayle, S. Beaird, C. Braud, J. Bonneson, P. Tarnoff, and T. Urbanik. Traffic Signal Timing Manual. Federal Highway Administration (FHWA), U.S. Department of Transportation, 2008.

Li, H., A. Hainen, C. Day, G. Grimmer, and J. Sturdevant. Longitudianl Performance Measures for Assessing Agency-Wide Signal Management Objectives. Transportation Research Record: Journal of the Transportation Research Board, No. 2355, Transportation Research Board of the National Academies, Washington, D.C., 2013, pp. 20-30.

List, G., B. Williams, N. Rouphail, L. Rodegerdts, K. Pincus, B. Nevers, and A. Karr. Establishing Monitoring Programs for Travel Time Reliability. SHRP 2 Report S2-L02RR-1, Strategic Highway Research Program 2 (SHRP 2), Transportation Research Board of the National Academies, 2014.

Liu, H., and J. Zheng. Automatic Generation of Traffic Signal Timing Plan. MN/RC 2014-38. Minnesota Department of Transportation, 2014.

Liu, H., and W. Ma. Time-dependent Travel Time Estimation Model for Signalized Arterial Network. Presented at 86th Annual Meeting of the Transportation Research Board, Washington, D.C., 2007.

Liu, H., W. Ma, X. Xu, and H. Hu. Development of a Real-Time Arterial Performance Monitoring System Using Traffic Data Available from Existing Signal Systems. MN/RC 2009-01. Minnesota Department of Transportation, 2008.

Liu, H., X. Wu, W. Ma, and H. Hu. Real-time Queue Length Estimation for Congested Signalized Intersections. Transportation Research Part C: Emerging Technologies, Vol. 17, No. 4, 2009, pp. 412-427.

Ma, W. A Real-Time Performance Measurement System for Arterial Traffic Signals. Dissertation, University of Minnesota, St. Paul, MN, 2008.

National Transportation Operations Coalition (NTOC). 2012 National Traffic Signal Report Card Technical Report. Federal Highway Administration (FHWA), U.S. Department of Transportation, 2012.

Petty, K., and T. Barkley. Arterial Performance Measurement in the Transportation Performance Measurement System (PeMS). Berkeley Transportation Systems, Inc. http://www.mtc.ca.gov/services/arterial_operations/downloads/pems_arterials_2011_05 10_v3.pdf. Accessed January 3, 2015.

Sharma, A., D. Bullock, and J. Bonneson. Input-Output and Hybrid Techniques for RealTime Prediction of Delay and Maximum Queue Length at Signalized Intersections. 
Transportation Research Record: Journal of the Transportation Research Board, No. 2035, Transportation Research Board of the National Academies, Washington, D.C., 2007, pp. 69-80.

Skabardonis, A., and N. Geroliminis. Real-Time Monitoring and Control on Signalized. Journal of Intelligent Transportation Systems: Technology, Planning, and Operation, Vol. 12, No. 2, 2008, pp. 64-74.

Smaglik, E., A. Sharma, D. Bullock, J. Sturdevant, and G. Duncan. Event-Based Data Collection for Generating Actuated Controller Performance Measures. Transportation Research Record: Journal of the Transportation Research Board, No. 2035,

Transportation Research Board of the National Academies, Washington, D.C., 2007, pp. 97-106.

Smaglik, E., D. Bullock, and A. Sharma. Pilot Study on Real-Time Calculation of Arrival Type for Assessment of Arterial Performance. Journal of Transportation Engineering, Vol. 133, No. 7, 2007, pp. 415-422.

Smaglik, E., D. Bullock, D. Gettman, C. Day, and H. Premachandra. Comparison of Alternative Real-Time Performance Measures for Measuring Signal Phase Utilization and Identifying Oversaturation. Transportation Research Record: Journal of the Transportation Research Board, No. 2259, Transportation Research Board of the National Academies, Washington, D.C., 2011, pp. 123-131.

Tan, P., M. Steinbach, and V. Kumar. Introduction to Data Mining. Pearson Addison Wesley, 2006.

Turner, S., W. Eisele, R. Benz, and D. Holdener. Travel Time Data Collection Handbook. FHWA-PL-98-035. Federal Highway Administration (FHWA), U.S. Department of Transportation, 1998.

$\mathrm{Wu}, \mathrm{X}$., and H. Liu. Using High-Resolution Event-based Detector Data for Traffic Modeling and Control: An Overview. Transportation Research Part C: Emerging Technologies, Vol. 42, 2014, pp. 28-43.

Zegeer, J., J. Bonneson, R. Dowling, P. Ryus, M. Vandehey, W. Kittelson, N. Rouphail, B. Schroeder, A. Hajbabaie, B. Aghdashi, T. Chase, S. Sajjadi, R. Margiotta, and L. Elefteriadou. Incorporating Travel Time Reliability into the Highway Capacity Manual. SHRP 2 Report S2-L08-RW-1, Strategic Highway Research Program 2 (SHRP 2), Transportation Research Board of the National Academies, 2014.

Zhang, H. Link-journey-speed Model for Arterial Traffic. Transportation Research Record: Journal of the Transportation Research Board, No. 1676, Transportation Research Board of the National Academies, Washington, D.C., 1999, pp. 109-115. 
Zheng, J., H. Liu, S. Misgen, and G. Yu. Performance Diagnosis Tool for Arterial Traffic Signals. Transportation Research Record: Journal of the Transportation Research Board, No. 2356, Transportation Research Board of the National Academies, Washington, D.C., 2013, pp. 109-116. 
VITA

\section{XUANWU CHEN}

$2001-2005$

B.S. Transportation Engineering

Southeast University, Nanjing, China

2005 - $2007 \quad$ Assistant Engineer

Guangzhou Municipal Engineering Design and Research Institute Guangzhou, China

$2007-2010 \quad$ M.S. Civil Engineering

University of Alaska, Fairbanks

2007 - $2010 \quad$ Graduate Research and Teaching Assistant

Alaska University Transportation Center

University of Alaska, Fairbanks

$2011-2012 \quad$ Instructor

Department of Civil and Environmental Engineering

Florida International University, Miami, Florida

$2011-2012$ Best Student Chapter Award, while serving as the Secretary, District 10 Institute of Transportation Engineers

2011 - $2016 \quad$ Doctoral Candidate in Civil Engineering

Florida International University, Miami, Florida

2011 - Present $\quad$ Student member, Institute of Transportation Engineers

2012 - $2013 \quad$ Outstanding Leadership Award, Council of Student

Organizations, Florida International University.

2012 - $2016 \quad$ Graduate Research Assistant

Lehman Center for Transportation Research

Florida International University, Miami, Florida

2012 - Present Member, Chi Epsilon Honor Society

$2013-2014 \quad$ President, Institute of Transportation Engineers (ITE) Student

Chapter, Florida International University

2013 - Present Student member, Women’s Transportation Seminar 
2014 - $2016 \quad$ Vice President, Women's Transportation Seminar (WTS)

Student Chapter, Florida International University

\section{PUBLICATIONS AND PRESENTATIONS}

Chen, X., M. Hadi, Y. Xiao, and L. Elefteriadou. Development of Macroscopic Emission Estimation Model based on Microscopic Operating Modes. Transportation Research Record: Journal of the Transportation Research Board No. 2570, Transportation Research Board of the National Academies, Washington, D.C., 2016, pp. 39-47.

Chen, X., and M. S. Lee. A Case Study on Multi-Lane Roundabouts under Congestion: Comparing Software Capacity and Delay Estimates with Field Data. Journal of Traffic and Transportation Engineering, Vol. 3, No.2, 2016, pp. 154165.

Hadi, M., X. Chen, H. Fartash, S. Khazraeian, and M. Iqbal. Data and Connected Vehicle Support of Active Traffic Management Strategies. Presented at T3e Webinar, U.S. Department of Transportation, 2016.

Chen, X., Y. Xiao, M. Hadi, M. Ackert, and P. S. Lin. Assessment of PrePreemption Strategy of Traffic Signals near Railroad Grade Crossing Utilizing Simulation Analysis. Presented at 93rd Annual Meeting of the Transportation Research Board, Washington, D.C., 2014.

Hadi, M., Y. Xiao, T. Wang, M. Iqbal, A. Massahi, J. Jia, X. Chen, and H. Fartash. Decision Support Systems for Transportation System Management and Operations (TSM\&O). Final Research Report, Florida Department of Transportation, Project BDV29-977-09, 2014.

Lin, P. S., Z. Wang, R. Guo, Q. Wang, S. Sunkari, P. Songchitruksa, L. Ruback, X. Chen, Y. Xiao, and M. Hadi. Coordinated Pre-Preemption of Traffic Signals to Enhance Railroad Grade Crossing Safety in Urban Areas and Estimation of Train Impacts to Arterial Travel Time Delay. Final Research Report, Florida Department of Transportation, Project BDK85 977-44, 2014. 